\title{
L'élaboration scripturale du francoprovençal au Moyen Âge
}

\author{
Carles, Hélène ; Glessgen, Martin-Dietrich
}

\begin{abstract}
Francoprovençal has generally been viewed as an oral dialect group with a highly varied character (due to the mountainous regions of the Alps, the Jura and the Massif Central), and with no elaborated written textual tradition. The virtual absence of such a tradition may indeed be observed for the modern period (i.e. the second half of the second millennium). However, this is not the case for the medieval period, during which Francoprovençal underwent a process of elaboration similar to that of the neighbouring Romance languages, at first fragmentary and embedded in Latin. From these beginnings emerged a pure Romance $<$ jats:italic $>$ scripta $</$ jats:italic $>$ which remained in existence until the end of the 15th century, when it was replaced by French. This process remained incomplete, characterised by the continuous copresence of either Latin or French and essentially limited to legal and administrative documents. Nonetheless, the first half of the second millennium yields a complex written vernacular which displays both diatopic and diachronic variation, and which has never before undergone systematic analysis. The present contribution is based on a corpus of medieval documents from the Francoprovençal regions of France and Switzerland (approx. 700.000 words), digitised as part of the project $<$ jats:italic $>$ Documents linguistiques galloromans $</$ jats:italic $>$ and presented for the first time here. Taking one of the most salient and frequent phenomena as an example - the outcome of lat. /a/ in tonic free syllables ( $<$ jats:sc $>$ pratu $</$ jats:sc $>$ gt; Frpr. $<$ jats:italic $>$ pra $<$ jats:italic $>$ vs Fr. $<$ jats:italic $>$ pré $</$ jats:italic $>$ ) - the authors assess the nature and extent of the presence of Francoprovençal in medieval scripturality, examining a total of 60.000 occurrences. The results thus obtained allow an interpretation of the historical development of Francoprovençal on the basis of entirely new evidence.
\end{abstract}

DOI: https://doi.org/10.1515/zrp-2019-0003

Posted at the Zurich Open Repository and Archive, University of Zurich ZORA URL: https://doi.org/10.5167/uzh-181968

Journal Article

Published Version

Originally published at:

Carles, Hélène; Glessgen, Martin-Dietrich (2019). L'élaboration scripturale du francoprovençal au Moyen Âge. Zeitschrift für romanische Philologie, 135(1):68-157.

DOI: https://doi.org/10.1515/zrp-2019-0003 


\title{
L'élaboration scripturale du francoprovençal au Moyen Âge
}

\author{
À la mémoire de Max Pfister, \\ le maître et l'ami
}

https://doi.org/10.1515/zrp-2019-0003

\begin{abstract}
Francoprovençal has generally been viewed as an oral dialect group with a highly varied character (due to the mountainous regions of the Alps, the Jura and the Massif Central), and with no elaborated written textual tradition. The virtual absence of such a tradition may indeed be observed for the modern period (i.e. the second half of the second millennium). However, this is not the case for the medieval period, during which Francoprovençal underwent a process of elaboration similar to that of the neighbouring Romance languages, at first fragmentary and embedded in Latin. From these beginnings emerged a pure Romance scripta which remained in existence until the end of the 15th century, when it was replaced by French. This process remained incomplete, characterised by the continuous copresence of either Latin or French and essentially limited to legal and administrative documents. Nonetheless, the first half of the second millennium yields a complex written vernacular which displays both diatopic and diachronic variation, and which has never before undergone systematic analysis. The present contribution is based on a corpus of medieval documents from the Francoprovençal regions of France and Switzerland (approx. 700.000 words), digitised as part of the project Documents linguistiques galloromans and presented for the first time here. Taking one of the most salient and frequent phenomena as an example - the outcome of lat. /a/ in tonic free syllables ('PRATU> Frpr. pra vs Fr. pré) - the authors assess the nature and extent of the presence of Francoprovençal in medieval scripturality, examining a total of 60.000 occurrences. The results thus obtained allow an interpretation of the historical development of Francoprovençal on the basis of entirely new evidence.
\end{abstract}

\footnotetext{
Adresse de correspondance : Prof. Dr. Hélène Carles, Université de Strasbourg, Faculté des Lettres, Le Portique, 14 rue René Descartes, BP 80010, F-67084 Strasbourg Cedex, E-Mail : hcarles@unistra.fr

Prof. Dr. Martin Glessgen, Universität Zürich, Romanisches Seminar, Zürichbergstr. 8, CH-8032 Zürich, E-Mail : glessgen@rom.uzh.ch
} 
Keywords : Francoprovençal, scripturality, language elaboration, Middle Ages, scripta, language contact, Old French, corpus linguistics, documentary texts, historical phonetics

Mots-clés : francoprovençal, scripturalité, élaboration linguistique, Moyen Âge, scriptae, contact linguistique, français ancien, linguistique de corpus, textes documentaires, phonétique historique

\title{
1 Acquis de la recherche et interrogations
}

\subsection{Le statut du francoprovençal médiéval}

La tradition de la recherche concernant le francoprovençal médiéval se scinde depuis longtemps entre deux points de vue complémentaires, mais aussi partiellement contradictoires: l'une prend appui sur l'idée que le francoprovençal représente essentiellement un ensemble dialectal qui n'a connu à aucun moment de "véritable » mise à l'écrit. Ce point de vue est synthétisé par Andres Kristol :

\begin{abstract}
«What we call 〈Francoprovençal〉 is not «a〉 language but a collection of speech varieties displaying a common linguistic typology yet an extremely high degree of dialect fragmentation. Francoprovençal has (or had) not one but several leading linguistic (micro-) centres (Lyon, Grenoble, Geneva, Fribourg, Sion, Aosta, etc.), each with very variable domains of influence ; [...] It has therefore never experienced any linguistic standardization. [...] Standardization is equally absent in writing. Even if the earliest documents date from the thirteenth century, Francoprovençal has never developed a common written tradition. It has always been essentially restricted to the domain of informal oral communication. [...] To a greater or lesser extent each author developed his own pratices in writing, generally on the basis of the writing conventions of French » (Kristol 2016, 350).
\end{abstract}

L'autre point de vue est celui d'une philologie médiéviste qui aborde les documents médiévaux du territoire francoprovençal sans se poser réellement la question du statut de la variété écrite en question. Cette approche suppose donc de manière implicite l'existence d'une mise à l'écrit, mais ne s'arrête pas sur les problèmes méthodologiques inhérents à sa situation pourtant bien différente de celle du français ou de l'italien médiéval, comme Kristol (2016) le rappelle à juste titre. Anne-Marie Vurpas a déjà formulé ce constat en 1995 :

«Le francoprovençal se présente paradoxalement comme une des régions dont les anciens textes sont les mieux connus, mais dont les études scriptologiques sont encore incomplètes et débattues. [...] On peut dire que le francoprovençal a [...] suscité de nombreuses études 
scriptologiques, à la fois chez les dialectologues suisses et français, sans toutefois [...] résoudre le problème fondamental que pose cette scripta ... »(Vurpas 1995, 389s.).

Dans la lignée d'A.-M. Vurpas, nous supposons en effet que le francoprovençal médiéval a bel et bien connu une élaboration scripturale, dans une logique certes non unitaire mais clairement supra-individuelle et que l'analyse linguistique des témoins anciens gagne à prendre en considération le scénario scriptural dans lequel ceux-ci s'inscrivent. Étant donné l'indéniable complexité de la situation, l'identification des lignes directrices demande une rigueur épistémologique particulière.

\section{2 Éléments généralement reconnus sur la trajectoire du francoprovençal médiéval}

Résumons brièvement les éléments qui peuvent être considérés comme acquis par la recherche :

(1) Le statut du francoprovençal comme «langue » romane à part entière ne fait pas de doute, malgré l'absence d'une élaboration à l'époque moderne et d'une unité géopolitique de son territoire. ${ }^{1}$ La délimitation géolinguistique du francoprovençal à l'époque moderne est également bien établie et la recherche s'accorde aussi à supposer une plus grande extension vers le Nord au Moyen Âge. ${ }^{2}$ Voici la carte établie par François Zufferey $(2006,457)$ dans cette optique : ${ }^{3}$

1 Cf. sur ce point la synthèse bien documentée de Stefania Maffei Boillat (2015, 45-53) : « L'espace francoprovençal au Moyen Âge» et «La 〈plus modeste» de 〈toutes les langues romanes» ", formulation qu'elle emprunte à Pierre Gardette $(1974,294)$.

2 Cf. Kristol (2004) et Chambon (2005).

3 L'idée de François Zufferey est d'illustrer «Le domaine francoprovençal dans son extension présumée vers $1300 »$. Nous serions plus prudents quant à la chronologie et enclins à supposer qu'il s'agit plutôt de l'extension vers l'An Mil. Les documents de Besançon et de Porrentruy du $13^{\mathrm{e}}$ siècle (cf. infra 2.3) plaident en effet davantage pour la présence d'une variété de type oïlique à l'oral déjà à cette époque. 


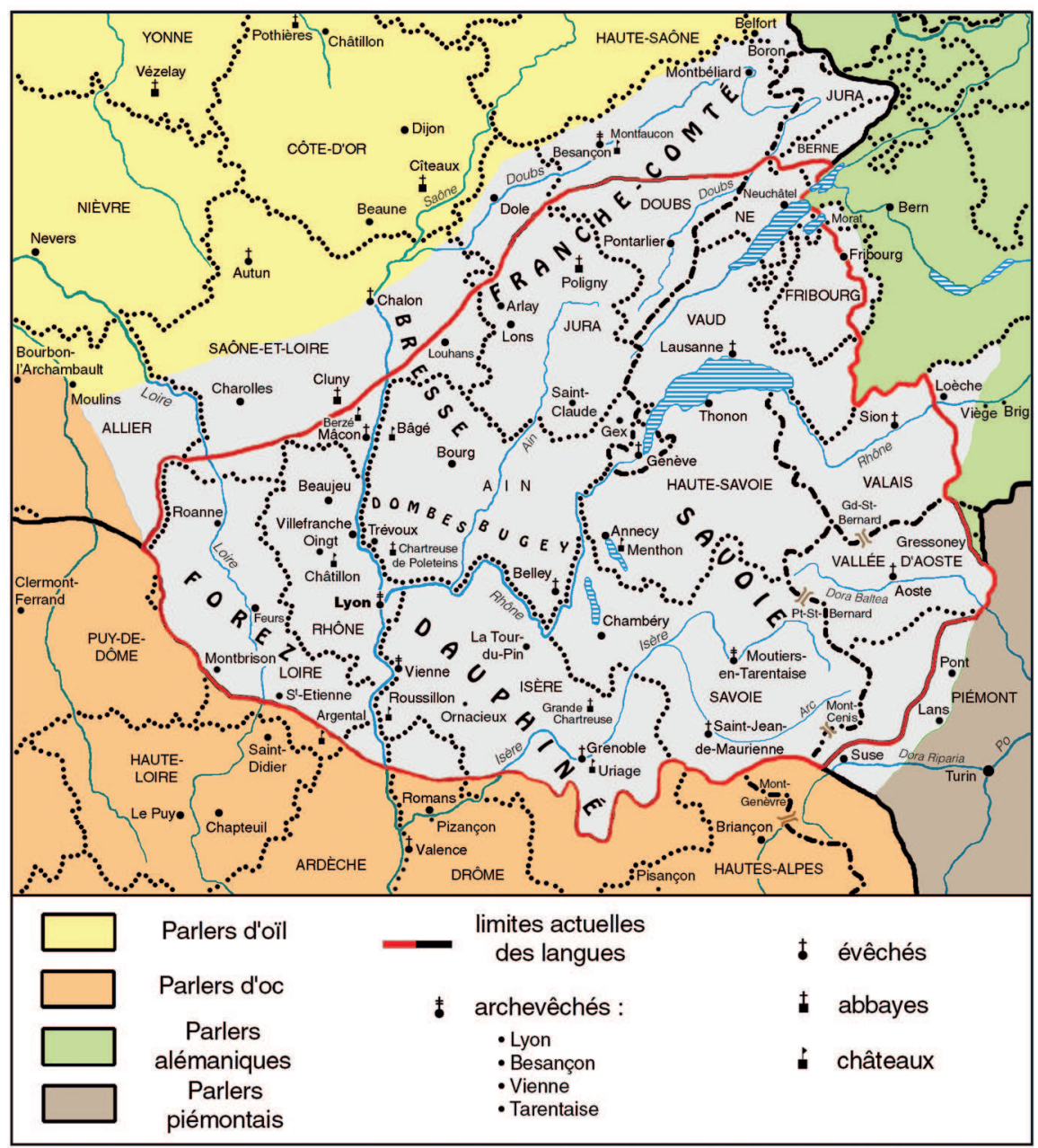

Ill. 1: Le domaine francoprovençal dans son extension maximale au Moyen Âge (@ F. Zufferey).

(2) Quant à la genèse du francoprovençal, nous retenons l'idée développée par Mgr Gardette (i) du rôle fédérateur de Lyon ${ }^{4}$ et (ii) de la constitution de son

4 Le rôle de Lyon a déjà été considéré comme essentiel par Heinrich Lausberg en 1956 (cité par Chambon/Greub 2008, cf. infra); Gardette (1974, 295-297) souligne son importance linguistique dans l'Antiquité tardive ; cf. aussi Schmitt $(1977,103)$ : « Lyon seul a façonné cette aire linguistique à toutes les époques. [...] Le francoprovençal [...] s'avère, comme l'a formulé Pierre Gardette, comme «unité sociale ancienne et toujours actuelles [1974, 304] groupée autour d'un centre de première importance »); cf. également l'argumentation nuancée de Chambon/Greub (2008, 
territoire le long des axes fluviaux - le Rhône (jusqu'au Valais) et « ses affluents », la Saône (anciennement bien au-delà de Mâcon en suivant aussi son affluent, le Doubs), l'Ain et l'Isère (jusqu'au Petit-Saint-Bernard) - longés par les voies routières qui mènent au-delà de la crête des Alpes vers le Val d'Aoste (à travers les deux cols Saint-Bernard, à partir du Valais au Nord et de l'Isère à l'Ouest). Autour de cette structure centrale, qui ressort très nettement de la carte de François Zufferey, le type linguistique a irradié vers les montagnes environnantes du Massif Central, du Jura et des Alpes. ${ }^{5}$

(3) Pour le Moyen Âge tardif, Gardette suppose que la langue a dû être moins éclatée qu'à l'époque moderne :

« aujourd'hui l'évolution phonétique, la disparition de tout centre directeur linguistique, la rencontre sur notre sol lyonnais des influences contradictoires du nord et du sud ont fait que nos patois nous donnent l'impression d'une grande diversité. Mais cette diversité, qui tient à des causes relativement récentes, n'existait sans doute pas, du moins n'était-elle pas aussi profonde au moyen âge, lorsque ces causes n'existaient pas encore » (Gardette 1974, 305).

Dans son idée, le francoprovençal a gardé une unité certaine jusqu'aux $16^{\mathrm{e}} /$ $17^{\mathrm{e}}$ siècles (ib.). Dans une logique parallèle, Vurpas $(1995,396)$ soutient que la période de prospérité de Lyon entre le milieu du $13^{\mathrm{e}}$ et la fin du $14^{\mathrm{e}}$ siècle a été déterminante dans le développement d'une scripta francprovençale.

(4) Pour poursuivre ce dernier aspect, qui est celui qui nous intéresse ici, le francoprovençal a en effet connu des manifestations écrites très apparentes au Moyen Âge, manifestations qui ont été éditées avec une grande attention et étudiées de près. Les textes plus ou moins proches d'un oral francoprovençal font apparition au milieu du $13^{\mathrm{e}}$ siècle. ${ }^{6}$ Ils sont présents jusqu'au milieu du $14^{\mathrm{e}}$ siècle. Ensuite les scriptae en territoire francoprovençal se francisent très nettement pour

2503-2506) ainsi que, pour les étapes de la formation du francoprovençal entre le $6^{\mathrm{e}}$ et le $12^{\mathrm{e}}$ siècle, Carles $(2017,185-187)$.

5 Il serait certainement porteur de mettre en lien, dans une approche transdisciplinaire, le contexte géopolitique évolutif du territoire francoprovençal avec ses trajectoires linguistiques. Dans les grandes lignes, on peut retenir que ce territoire se trouvait quelque peu à la périphérie des grands centres du pouvoir politique européen. Il s'inscrit toutefois avec une certaine cohérence dans le royaume mérovingien de Bourgogne entre le $6^{\mathrm{e}}$ et le $8^{\mathrm{e}}$ siècle (cf. aussi Zufferey 2006, 466), puis, sous l'Empire germanique, le royaume de Bourgogne et d'Arles entre le $10^{\mathrm{e}}$ et le $13^{\mathrm{e}}$ siècle. Le comté de Bourgogne (= la Franche-Comté), la maison de Savoie ou le duché de Bourgogne étaient parmi les acteurs principaux dans cette région que le royaume de France ne put atteindre que tardivement (notamment par le rattachement de Lyon à la couronne par Philippe le Bel entre 1292 et 1320).

6 Cf. Gardette (1968, 85s.) : «Les plus anciens textes dialectaux non littéraires apparaissent en Lyonnais vers 1250 [...] et 1260 [...], en Forez en 1289 [...]. » - V. infra chap. 2. 
devenir pleinement oïliques au $15^{\mathrm{e}}$ siècle. $^{7}$ Le francoprovençal disparaît alors définitivement de l'écrit non folklorique et nourrit le français régional présent dans les textes de l'époque moderne.

(5) Enfin, on relèvera que les manifestations écrites du francoprovençal (i) restent essentiellement circonscrites au domaine documentaire, comme c'est également le cas du gascon, du sarde ou du frioulan, et (ii) qu'elles se développent pratiquement toujours en dialogue avec l'écrit français dont la présence est plus ou moins marquée. ${ }^{8}$ La recherche parle d'une scripta " para-francoprovençale » - c'est ici la terminologie de Gossen (1970) - ou encore de scripta « francoprovençalisante » pour reprendre celle de Maffei Boillat (2015).

Curieusement, la recherche en sociolinguistique historique consacrée aux questions de plurilinguisme ne s'est que trop peu attardée sur le domaine francoprovençal au Moyen Âge. Les deux articles de Lüdi sur Fribourg (1985; 1989) mettent certes en avant la question du plurilinguisme dans cette ville, mais se concentrent sur les contacts de langue dans le triangle latin - dialecte germanique - dialecte francoprovençal, et non sur la relation entre français et francoprovençal. Aussi, nous ne connaissons à ce jour aucune recherche ayant mis en relief une élaboration scripturale dans un contexte réunissant deux langues galloromanes.

Une fois ces éléments réunis, les certitudes se raréfient. Si l'existence d'une scripta francoprovençale est reconnue, au moins par certains auteurs, elle n'a jamais été étudiée de manière systématique dans ses manifestations non littéraires, de loin les plus nombreuses, et encore moins en comparant les différents

7 Cf. Gardette $(1968,84)$ : « Après 1350, l'influence du français se fait sentir [à Lyon] et bien vite, on passe de textes dialectaux [= francoprovençaux] francisés à des textes français parsemés de formes et de mots de dialecte » (cf. aussi ib. 86) ; puis Durdilly $(1972,376):$ « [...] l’usage du dialecte dans la langue écrite a été constant pendant la première moitié de ce siècle $\left[=14^{\mathrm{e}} \mathrm{s}\right.$.] et même un peu au-delà, jusqu'à 1360 environ, mais [...] le français a réussi à s'imposer ensuite, dans les quinze dernières années tout au moins ». Elle renvoie à Brun $(1923,62)$ qui fournit des éléments complémentaires et conclut « dans les trente dernières années du XIV siècle, le français devient assez commun [...]. Au XV siècle, le français est généralisé ». Quant au Dauphiné, Devaux (1892, 14) constate que dès 1333 « les traces du dauphinois dans les textes français se font de plus en plus rares ». - Ces indications sont confirmées par notre analyse, v. infra chap. 6.1.

8 L'interférence entre les deux systèmes linguistiques est bien apparente dans le domaine des rares textes non documentaires réunis de manière exhaustive par Stefania Maffei Boillat (2015, 45-61) : elle nomme, à côté de seulement trois ensembles rédigés dans une scripta francoprovençale (un légendaire en prose, étudié nouvellement plus en détail par Maffei [cf. aussi Stimm 1955], le recueil des écrits de Marguerite d'Oingt et la Somme du Code, cette dernière étant intégrée à notre corpus, cf. infra 2.2 et 8.2) une douzaine de textes littéraires qui connaissent une scripta mixte (à commencer par Girart de Roussillon), comportant des éléments francoprovençaux dans une mesure très variable. 
domaines de part et d'autre du Jura et des Alpes. Il n'existe aucune synthèse, aucune vue d'ensemble qui établirait les trajectoires de l'écrit francoprovençal au Moyen Âge et qui décrirait la physionomie de sa scripta. Aussi, sa nature et son statut langagier et sociolinguistique n'ont jamais été précisés d'un point de vue systémique et ses dynamiques évolutives sont restées opaques. Le statut même des textes francoprovençaux n'est pas bien cerné et les auteurs évoquent à loisir l'incompétence des scribes pour expliquer la présence de cette langue à l'écrit.

Voici quel fut le point de départ de la réflexion que nous développerons par la suite, en présentant tout d'abord un certain nombre de thèses et points de discussion (1.3), puis les objectifs concrets de notre recherche et de la présente étude (1.4). ${ }^{9}$

9 La présente étude repose sur un travail de longue haleine qui implique, de manière complémentaire, ses deux auteurs ; pour ne pas compliquer la lecture, le pronom « nous » désigne ainsi par la suite, à tour de rôle, l'un(e) d'entre nous ou nous deux. Nous avons également pu nous valoir de l'apport de nombreux jeunes chercheurs et collègues que nous souhaiterions remercier très sincèrement en ce lieu. Ainsi, le repérage et le balisage scriptologique, qui prennent appui sur les travaux préparatoires à l'étude de Glessgen (2008), ont été élargis à Zurich par Dumitru Kihaï (2011) et Sarah Tinner (ms. 2009, 2011), puis par Riccarda Bürli (2017), avant d'être nouvellement repris et élargis par nous deux ; le répertoire bibliographique sur le francoprovençal médiéval a été réuni entre 2011 et 2014 par Elisabeth Berchtold et Laure Grüner alors doctorantes FNS à Neuchâtel et à Nancy, sous la direction d'Andres Kristol et de Yan Greub; la numérisation et la mise en ligne des textes du territoire francoprovençal ont été réalisées en 2016 sous notre direction commune et préparées par Lorraine Fuhrer et Aurélie Reusser-Elzingre à Neuchâtel ; l'identification d'une série de paramètres grapho-phonétiques et morphologiques pertinents et de la bibliographie les concernant a été menée à bien de nouveau par Lorraine Fuhrer qui prépare sa thèse sur la scripta particulière de Fribourg et qui est notre principale collaboratrice dans le projet FNS intitulé «L'élaboration scripturale du francoprovençal au Moyen Âge » ( $n^{\circ}$ 100012_172826, financé entre février 2018 et janvier 2021). Nous avons également reçu un soutien précieux de plusieurs informaticiens, Samuel Läubli, Mathias Müller et Linus Manser à Zurich, Marcus Husar à Heidelberg et Pierre Ménetrey à Neuchâtel. Nous sommes par ailleurs reconnaissants de l'aide financière de la Fondation Pierrehumbert et de l'Université de Neuchâtel pour la numérisation du corpus francoprovençal ainsi que de l'apport du Fonds National Suisse dont le soutien financier nous permettra de mener à bien avec Lorraine Fuhrer ainsi qu'avec Dumitru Kihaï les analyses scriptologiques et lexicologiques du corpus en question. Nous remercions également, pour leur relecture attentive du présent article, Elisabeth Berchtold, Jean-Pierre Chambon, Jean-Paul Chauveau, Lorraine Fuhrer, Yan Greub et Paul Videsott.

Qu'il nous soit permis enfin de formuler une dernière pensée pour le très regretté Max Pfister qui, le premier, a accueilli la présentation de notre projet lors du Congrès de Linguistique et de Philologie Romanes à Rome en juillet 2016 avec un enthousiasme et une bienveillance desquels il avait le secret et qui nous ont très fortement encouragés dans la poursuite de cette recherche. Nous regrettons qu'il n'ait pas pu prendre connaissance de l'avancée de nos travaux qu'il voyait comme un lointain écho de son monumental Girart de Roussillon. 


\subsection{Thèses et points de discussion autour des trajectoires évolutives du francoprovençal écrit au Moyen Âge}

\subsubsection{L’époque pré-textuelle}

Un premier élément qui n’a jamais été considéré dans ce contexte concerne la phase préparatoire de l'émergence du francoprovençal à l'écrit. L'élaboration scripturale du francoprovençal se place en effet dans un premier temps, comme celle des autres idiomes romans, dans le cadre du latin médiéval. Dès les $9 \%$ $11^{\mathrm{e}}$ siècles, des éléments lexicaux et infra-lexématiques vernaculaires sont progressivement insérés en contexte latin par les scribes. Nous avons pu prouver cette présence de lexèmes francoprovençaux à date ancienne (Carles 2017) ${ }^{10}$ et nous avons également pu montrer pour l'Auvergne que ces insertions résultent d'un phénomène d'élaboration systématique de la langue vernaculaire sous le manteau du latin normatif (Carles 2011). Ces éléments francoprovençaux fragmentaires restent ensuite présents dans les textes latins jusqu'au $16^{\mathrm{e}}$ siècle. $^{11}$

Or, cette élaboration a préparé de manière organique dans les autres régions galloromanes la mise à l'écrit de ces mêmes langues vernaculaires sous forme de scriptae régionales (picarde, languedocienne etc.). Elles connaissent alors aux $12 \mathrm{e}$ $13^{\mathrm{e}}$ siècles une élaboration systématique caractérisée par une autonomie scripturale croissante. Si dans la phase préparatoire, le francoprovençal s'inscrit dans la même logique que les langues romanes voisines, il emprunte par la suite une voie différente. Plus précisément, l'écrit francoprovençal choisit deux voies parallèles, l'une sur le territoire de la Suisse actuelle, l'autre sur celui de la France actuelle, ${ }^{12}$

10 Nous renvoyons ici à notre Trésor galloroman des origines (TGO, Carles 2017) qui traite sous une forme lexicographique détaillée 48 lexèmes francoprovençaux relevés en contexte latin entre ca 800 et 1120 dans les Chartes originales latines conservées en France (corpus de l'ARTEM).

11 L'importance du triangle latin-francoprovençal-français a déjà été identifiée par Devaux (1892, 17 ; 21), puis soulignée par Aebischer $(1950,6)$ et Gardette (1968, 71s.). Pour la Suisse romande, l'excellente monographie de Vitali (2007) met en lumière la présence significative du vocabulaire francoprovençal en contexte latin aux $12^{\mathrm{e}} / 15^{\mathrm{e}}$ siècles.

12 Nous parlerons par la suite simplement de «Suisse» et de «France» pour désigner les territoires de la Suisse romande et de la France actuelles séparés géographiquement par les massifs du Jura et des Alpes. Il est en effet difficile d'étiquetter les différents ensembles. Comme nous l'avons déjà dit (supra, n. 4), les rattachements géopolitiques des différentes régions du territoire francoprovençal ont été soumis à des changements importants à travers les siècles. Le territoire de la Suisse romande était intégré, au Moyen Âge, dans le Comté de Bourgogne impérial, alors que les territoires francoprovençaux de la France actuelle étaient géopolitiquement parlant plus éclatés. 
tandis qu'en Val d'Aoste il reste confiné à un emploi fragmentaire en contexte latin. $^{13}$

Il est important de souligner que cette évolution bipartite ne fait surface qu'à l'époque où se développent les textes vernaculaires pleins. Aussi ce passage se place en domaine francoprovençal plus tard qu'en Picardie ou en Languedoc, mais il reste en phase avec d'autres régions galloromanes comme la Gascogne ou la Bourgogne. Entre le $9^{\mathrm{e}}$ et le $13^{\mathrm{e}}$ siècle, le territoire francoprovençal ne se distingue donc en rien de ses voisins, ni par les modalités de son élaboration en contexte latin ni par le moment où il développe une scripturalité vernaculaire pleine.

\subsubsection{La bipartition dans l'élaboration du francoprovençal}

La bipartition des trajectoires du francoprovençal est un deuxième élément interprétatif qui mérite d'être mis en lumière. Il ressort en effet de manière évidente de notre étude quantificatrice qu'en Suisse, l'écrit vernaculaire se place depuis le $13^{\mathrm{e}}$ siècle dans la logique d'une scripta française importée. Les modèles d'une scripta oïlique, souvent de type sud-oriental, sont utilisés par les rédacteurs et scribes de langue francoprovençale, tout en introduisant des éléments graphophonétiques, morphologiques et lexicaux de leur langue maternelle. ${ }^{14} \mathrm{Au}$ moment où le prestige sociolinguistique des langues vernaculaires augmente, le français prend donc la place auparavant occupée par le latin en tant que variété écrite acrolectale tandis que la langue autochtone basilectale - le francoprovençal - poursuit presque exclusivement une élaboration scripturale de type fragmentaire. Le cadre dans lequel se place le francoprovençal médiéval de Suisse entre le $13^{\mathrm{e}}$ et le $15^{\mathrm{e}}$ siècle est donc dans son ensemble semblable à celui de l'époque pré-textuelle antérieure $\left(9^{\mathrm{e}}-12^{\mathrm{e}} \mathrm{s}\right.$.).

En France se produit en revanche, comme nous le verrons, une élaboration scripturale plus intense du francoprovençal, surtout entre le milieu du $13^{\mathrm{e}}$ et le

13 Retenons à ce sujet la formulation de Schüle $(1975,341)$ : « Les phrases sont pensées en langue vulgaire. Le fond de la scripta savoyarde du XV siècle est un ensemble composite formé d'éléments francoprovençaux (localisables parfois en Valais, en Vallée d'Aoste ou en Savoie) et français, habillés et traduits en latin» (italiques dans l'original). Ernest Schüle est l'un des rares auteurs qui perçoit avec autant de clarté que les éléments vernaculaires ne sont pas l'effet d'une corruption du latin, mais celui d'une latinisation à partir de la langue parlée.

14 Cf. Gossen $(1970,346)$ : « il s'agit plutôt d'une scripta «bourguignonne» sur laquelle viennent se greffer des francoprovençalismes »; puis : « les scribes devaient être du pays, mais ils étaient sans doute formés à l'école «bourguignonne» ou se tenaient à des formulaires «bourguignons` ». 
milieu du $14^{\mathrm{e}}$ siècle. Celle-ci se rapproche des processus observables dans les langues romanes voisines, même si elle reste particulière à cause de la présence d'éléments français dans la scripta francoprovençale. La part du français peut être fortement réduite dans l'apparence textuelle, même si la coprésence de deux systèmes linguistiques caractérise aussi la trajectoire du francoprovençal écrit en France. Ce dernier forme ainsi au Moyen Âge un ensemble physionomique très disparate et difficile à appréhender sans une réflexion épistémologique concernant le phénomène de scripta mixte. Ses manifestations concrètes se (dé)placent sur un large continuum compris entre deux pôles, celui du français d'une part et du francoprovençal d'autre part.

Si ces observations ne sauraient surprendre les spécialistes du francoprovençal, elles n'ont jamais été dégagées de cette manière pour ensuite soumettre les différentes manifestations écrites à des analyses systématiques. La raison essentielle en est que les travaux interprétatifs sur le francoprovençal médiéval, somme toute peu nombreux comparés à ceux portant sur le domaine d'oïl, se concentrent pour l'essentiel sur un seul des deux territoires nationaux. C'est le cas, pour la France, des travaux fondamentaux de Gardette (1962; 1968; 1974), ${ }^{15}$ tout comme, pour la Suisse, des études méthodologiques réunies dans Marzys/Voillat (1971), de Marzys (1978) ${ }^{16}$ et des analyses minutieuses de la scripta helvétique de Gossen (1970) et de Müller (1981; 1990; 1997; 2001) qui mettent notamment en relief les éléments francoprovençaux identifiables, mais sans les placer dans le contexte général de la langue au Moyen Âge. ${ }^{17}$ C'est également le cas, pour l'Italie, de l'aperçu diachronique de Schüle (1978).

Des études qui portent sur l'ensemble du domaine telles que les réflexions de Helmut Stimm (1952) et de Christian Schmitt (1977) sur la genèse du francoprovençal restent peu fréquentes. ${ }^{18}$ La seule analyse linguistique interne qui englobe les deux domaines est celle de phonétique historique de Hans Hafner, qui a intégré dans sa version définitive (1955) les apports de Stimm; cette étude, certes datée et souffrant de l'absence de contrastivité avec le domaine oïlique, reste excellente par son emprise systémique et systématique du francoprovençal médiéval. La seule synthèse globale sur la tradition de la recherche est celle de Vurpas (1995). Ajoutons que Hafner ne commente aucunement la différence de nature entre les

15 Cf. aussi les analyses morphologiques ponctuelles de Bengt Hasselrot (1966) et de Brigitte Horiot $(1971 ; 1972)$.

16 Cf. aussi pour la Suisse Aebischer (1950) ou Kristol (2003; 2004).

17 Ainsi, Gossen (1970) étudie cinq textes de Genève (datés entre 1284 et 1310) en établissant une typologie scripturale du francoprovençal médiéval, mais sans mentionner les différences entre les textes de Suisse et de France.

18 Ce n'est sans doute pas le fait du hasard que ces deux auteurs ne soient ni français ni suisses. 
scriptae de France et de Suisse et que Vurpas l'évoque de manière très succincte (1995, 395 ; cf. 390-392).

Les travaux disponibles se placent ainsi généralement dans un cadre national voire local. Le cas habituel correspond à des études consacrées à de petits ensembles textuels propres à une région. De telles approches ciblées restreignent la portée et l'impact des études sur une langue dont le territoire se partage aujourd'hui entre trois pays voisins.

\subsubsection{La conscience linguistique}

L'absence d'emprise globale sur le francoprovençal médiéval est sans doute liée, au moins en partie, au fait que le phénomène de son élaboration scripturale n'est pas véritablement admis en tant que tel. L'idée ancienne selon laquelle les éléments francoprovençaux dans les textes médiévaux seraient dus à une incapacité des scribes se relève non seulement chez Devaux (1892), Jeanjaquet (1905), Duraffour (1925) ou Aebischer (1950), mais encore chez Gonon (1974) ou Marzys (1978) :

«Le parler local n'apparaît [...] que dans la mesure où le laisser-aller des scribes ou leur connaissance insuffisante du français lui permettent de s'infiltrer » (Jeanjaquet 1905, 272).

« [...] chartes rédigées par un scribe qui n’avait que de vagues lueurs sur la langue littéraire [...]. De tout temps, le franco-provençal a été un pis-aller ; et ce n'est pas d'hier non plus qu'il sert surtout à faire rire » (Aebischer 1950, 5).

« [...] c'est, à vrai dire, un charabia digne du «Malade Imaginaire` ou du Mamamouchi : il semble cependant bien nécessaire de recueillir ce charabia!» (Gonon 1974, XX ; cf. aussi XVI) ${ }^{19}$

Seul Wulf Müller (1981) s’oppose de façon explicite à cette tradition, en reprenant les termes de Jeanjacquet ; selon lui,

« les éléments caractéristiques de cette scripta sont dus, non à un laisser-aller hypothétique des scribes, mais à la volonté des Fribourgeois médiévaux de se créer des institutions bien à eux » (Müller 1981, 77).

Il faut dire que l'argument de l'incapacité des scribes médiévaux est endémique dans la tradition philologique du $20^{\mathrm{e}}$ siècle ${ }^{20}$ et qu'il a seulement pu être dépassé par les acquis des deux ou trois dernières décennies concernant l'élaboration linguistique et scripturale au Moyen Âge. Catalysée et cadrée notamment par le

19 Cf. aussi Devaux (1892, 20-26); Duraffour $(1925,14)$.

20 Cf. Carles $(2011,4$; 2017, 8). 
projet des ScriptOralia (139 vol., 1987-2013), l'interprétation des processus de scripturalité a pu prendre appui sur les concepts de proximité-distance et du diasystème (cf. Koch 1993), sur la structuration systématique des paramètres de l'histoire externe (cf. RSG 2003-2008; Glessgen 2012a) tout comme sur le développement de la sociolinguistique historique médiéviste. ${ }^{21}$

Étant donné que la tradition d'études sur le francoprovençal médiéval se place pour l'essentiel avant ce changement de paradigme, les prises de position antérieures sont restées dans un sens non contredites. Le fait est problématique puisque la question de l'incapacité des scribes entraîne celle de la conscience linguistique, pour laquelle la recherche distingue deux aspects : (i) le fait que les locuteurs et les scribes du francoprovençal aient considéré leur idiome différent ou non du français (et de l'occitan), puis, en cas positif, (ii) la question de savoir si les mêmes personnes considéraient que leur langue avait une unité à travers tout le territoire où elle était parlée.

Supposer une incompétence de fond des scribes en revient déjà à nier la première idée :

« [...] on admettra que le francoprovençal, au Moyen Age, n'est pas une «troisième langue» gallo-romane à côté du français et du provençal, si par «langue» on entend un code écrit bien caractérisé et distinct des autres. La scripta francoprovençale, quels que fussent ses caractères spécifiques, ne paraît être qu'une variante subordonnée du français, de valeur culturelle inférieure, au même titre que les différentes scriptae de langue d'oïl » (Marzys 1978, 204).

« nous ne connaissons, à époque ancienne, aucun témoignage explicite d'une distinction consciente entre francoprovençal et français » (Marzys 1978, 197).

Cette idée a toutefois déjà été contredite par Gardette, qui a défendu la conscience de l'altérité du francoprovençal par rapport au français et à l'occitan et a supposé aussi, au moins dans le territoire de la France, la conscience d'une unité linguistique francoprovençale «assez forte pour être opposée à l'unité française et à l'unité provençale » $(1968,87$ s.). Le concept d'« unité » est ici quelque peu perturbant puisqu'il ne semble pas englober, chez Gardette, le territoire de la Suisse. S'il nous semble en effet difficile de plaider une véritable conscience d'unité du francoprovençal - qui est également absente, par exemple, auprès de la majorité des locuteurs modernes de l'occitan -, la conscience de son altérité ressort de plusieurs observations de divers ordres.

21 Après les travaux précurseurs de Romaine (1982; 1988) dans la recherche anglo-saxonne, cf. Lodge (2004; 2009), Aquino-Weber/Cotelli/Kristol (2009) et notamment Lusignan (2012) qui combine dans son étude de la scripta picarde les approches philologique, sociolinguistique et historique. - Cf. aussi la réflexion nuancée de Vitali (2007, 93-117) sur l'interférence latinfrancoprovençal dans une clé de diglossie et de code-switching. 
Ainsi, Stefania Maffei Boillat (2015, 48s.) rappelle le témoignage bien connu d'Aimon de Varenne dans Florimont (v. 13614-13620), qui, au-delà du motif littéraire qu'il représente, a permis à la recherche d'entrevoir une perception spécifique de la langue propre à cet auteur (Tuaillon 2001; Zufferey 2006).

Gaston Tuaillon ajoute un indice complémentaire :

« La différence linguistique entre la langue d'oïl et le francoprovençal a sans doute empêché que la culture des trouvères ne rayonne jusqu'à Lyon et Genève. Cette rupture linguistique était bien ressentie par les personnes cultivées qui parlaient francoprovençal » (Tuaillon 2001, 31).

L'argumentation la plus complète se trouve chez Wulf Müller qui défend l'« existence d'une conscience linguistique chez ceux qui se servaient de la scripta fribourgeoise » $(1981,77)$; en parlant des scribes de Fribourg il argumente que

« leur formation intellectuelle et leurs expériences (voyages en France, voyageurs français à Fribourg) les rendaient certainement capables de voir les différences entre le français de Paris (ou de Bourgogne) et le francoprovençal de Suisse. Il ne leur était pas difficile, probablement, de constater qu'il s'agissait là de deux variétés parfaitement distinctes. D'ailleurs, la situation traditionnelle de bilinguisme à Fribourg ne pouvait avoir pour effet général que de renforcer la perception des phénomènes linguistiques. Pareillement, les quelques traits dialectaux de la scripta fribourgeoise ne pouvaient pas simplement passer inaperçus. Si on les avait introduits et maintenus dans le médium écrit, c'était de façon délibérée, parce qu'on avait pleinement conscience d'avoir une personnalité bien à soi et parce qu'on en était fier, fierté justifiée par la richesse et la quasi-indépendance de la ville » (ib., 80s.).

D'un point de vue de l'historien, Philippe Marguerat insiste également sur le rôle des facteurs externes comme le niveau de formation des chanceliers à Fribourg, l'essor économique de la ville, l'ascension politique de la bourgeoisie et établit, justement, un lien entre la qualité de la formation des scribes et l'introduction du francoprovençal à l'écrit, et non l'inverse (1971). ${ }^{22}$

Il est enfin possible de prendre appui sur la trajectoire pré-textuelle pour rendre plausible la conscience d'une diglossie chez les scribes et auteurs des documents. Rappelons que ce sont les mêmes scribes qui insèrent dans les textes documentaires latins, encore nombreux aux $13^{\mathrm{e}}$ et $14^{\mathrm{e}}$ siècles, des éléments vernaculaires francoprovençaux. Il ne peut pas y avoir de doute qu'ils sont alors bien conscients qu'il s'agit d'une interférence entre deux langues distinctes (cf.

22 Le raisonnement de Müller et de Marguerat s'oppose ainsi à l'idée que le choix du francoprovençal aurait été un choix par défaut : «La langue locale, encore en usage en 1971, a toujours été et reste de petit renom pour tout ce qui s'estime savant, décrassé de la paysannerie » (Gonon 1974, xxv ; cette idée est reprise telle quelle par Vurpas 1995, 394). 
Vitali 2007; Carles 2017). D’autant plus que l'on relève en contexte latin systématiquement des lexèmes francoprovençaux et jamais français, ce qui montre bien que les scribes ont une perception précise de ce qui est leur langue maternelle.

En remplaçant le latin par le français comme langue-« support », la tradition de l'insertion des éléments de langue maternelle est poursuivie, ce qui produit alors la scripta que nous connaissons avec des degrés de mixité souvent plus avancés et avec une plus grande diversité dans ses manifestations concrètes. Cela s'explique par le fait que l'écart autant linguistique interne que sociolinguistique séparant le francoprovençal du français était moins important que celui entretenu avec le latin. L’interaction entre les deux langues galloromanes était par conséquent plus aisée. ${ }^{23}$

Nous n'avons aucune raison de douter que dans la conscience des scribes, la langue d'oïl et le francoprovençal appartenaient à deux systèmes linguistiques clairement distincts. La proximité interne des variétés dialectales francoprovençales et oïliques, le cas échéant franc-comtoises, ne réduit en rien cette conscience. $^{24}$

\subsubsection{Première synthèse et perspectives}

Sur la base de ces premières réflexions, il nous semble dorénavant raisonnable de placer l'émergence du francoprovençal à l'écrit dans l'optique d'une élaboration scripturale consciente et non dans celle d'un malentendu auquel auraient été sujets quelques centaines de scribes incompétents à l'œuvre pendant plusieurs siècles. Le phénomène des scriptae mixtes plus ou moins francoprovençalisantes reste bien entendu transitoire et confiné au Moyen Âge tardif, avec une apogée entre le milieu du $13^{\mathrm{e}}$ et le troisième quart du $14^{\mathrm{e}}$ siècle, moment où le français pré-standardisé s'impose comme langue écrite autant sur le territoire de la France

23 Il serait intéressant de comparer cette configuration avec les cas de figure concrets observables en domaine occitan au moment où le français a exercé une pression grandissante à l'écrit (cf. Chambon/Olivier 2000). L'on constatera sans doute des ressemblances, même si l'époque est plus tardive, que les rapports de force sont différents et que le français se greffe alors sur une langue écrite, élaborée auparavant, et non l'inverse.

24 Il nous semble par conséquent important de nuancer la considération de Chambon/Greub (2008, 2513) selon lesquels «le domaine francoprovençal ne forme pas, sociolinguistiquement parlant, un ensemble séparé de l'oïl ; très tôt le français y fonctionnera comme langue-toit », en raison de l'absence d'« indice d'une conscience [...] d'une unité spécifique du francoprovençal ». La conscience de l'unité n'est pas nécessaire pour la conscience de l'altérité et nous verrons par la suite qu'au moins avant la fin du $14^{\mathrm{e}}$ dans le Lyonnais le français ne fonctionne pas encore comme «langue-toit» pour le francoprovençal. 
que de la Suisse, à l'instar de ce qui se passe un peu plus tard dans le domaine d'oc. Notre analyse permettra de préciser la chronologie et les trajectoires internes de cette élaboration scripturale qui a été avortée relativement tôt sous la pression croissante du français. Il s'agit ainsi d'un épisode d'élaboration intense et court, ce qui ne réduit en rien son intérêt pour l'histoire et la théorie linguistiques, mais qui pourrait expliquer pourquoi la recherche n'a jamais étudié en profondeur ce phénomène aussi singulier que significatif.

D'un point de vue des traditions de la recherche, nous pouvons retenir que les travaux sur la langue de l'écrit documentaire du domaine francoprovençal voient le jour dès la fin du $19^{\mathrm{e}}$ siècle et qu'ils ont connu une densité notable tout au long du $20^{\mathrm{e}}$ siècle, mais aussi qu'ils n'ont de loin pas eu le même essor au début du $21^{\mathrm{e}}$ siècle que les travaux sur la langue d'oïl. On constate notamment l'impasse concernant les avancées technologiques des deux dernières décennies. À ce jour, les données linguistiques n'ont jamais été quantifiées et traitées dans une optique structurant les réseaux scriptologiques du francoprovençal. C'est d'autant plus regrettable que le francoprovençal est devenu par là le parent pauvre de la Galloromania voire de la Romania, à l'heure où la linguistique médiéviste sur base de corpus a connu un développement considérable.

\subsection{Objectifs de notre recherche et de la présente étude}

Pour pouvoir exploiter le potentiel empirique et interprétatif qui réside dans la matière du francoprovençal médiéval, nous avons envisagé la constitution d'un corpus électronique fiable sur une base géo-chronologique et quantitative large permettant des analyses systématiques dans la logique de la linguistique de corpus. Nous avons ainsi intégré un volume textuel significatif provenant du territoire francoprovençal de Suisse et de France, dans la base de données des Documents linguistiques galloromans, ce qui garantit en même temps la contrastivité avec les scriptae oïliques, indispensable autant pour l'analyse interne que sociolinguistique. Ce corpus ne comporte pas de nouvelles éditions, à l'exception des documents de Fribourg (éd. Fuhrer en cours), mais sa constitution et son intégration dans les Documents linguistiques crée dès à présent une nouvelle visibilité à la mise à l'écrit médiévale du francoprovençal. Nous présenterons par la suite les relevés et réflexions qui ont présidé à ce travail de sélection et de numérisation (chap. 2).

Ce corpus fournit en même temps une base, aussi fiable que nouvelle, pour étudier très en détail le « jeu des possibles » de l'écrit en territoire francoprovençal et de cerner les trajectoires de son élaboration scripturale au Moyen Âge, sujet qui réserve un certain nombre de surprises. L'approche quantificatrice permet de 
dépasser les impressions laissées par une lecture en surface afin de déceler les logiques langagières véritablement à l'œuvre.

Nos objectifs sont de décrire ce processus d'élaboration dans le triangle entre latin, français et francoprovençal et de mettre en relief ses modélisations concrètes. D'un point de vue de l'étude du francoprovençal, cela permettra aussi de mettre en évidence les manifestations jusqu'ici jamais identifiées d'une conscience linguistique auprès des acteurs de l'écrit médiéval. Dans le domaine des Medieval Literacy Studies, cela permettra de mieux comprendre les processus en jeu lors de l'élaboration des autres scriptae romanes, notamment par la comparaison avec la langue d'oïl voisine. L'exemple francoprovençal jette une lumière nouvelle sur l'utilisation de modèles écrits pré-existants et sur les effets de la conscience linguistique dans l'élaboration d'une langue écrite.

Notre projet comporte deux volets qui répondent à une double lacune dans l'état actuel de la recherche sur le francoprovençal médiéval :

(1) Sur la base du corpus numérisé, nous avons entrepris, avec Lorraine Fuhrer, une analyse scriptologique qui entend exploiter, à travers le traitement empirique, le potentiel interprétatif pour la modélisation de l'élaboration scripturale et linguistique du francoprovençal. Nous préciserons ce volet du projet par la suite en l'illustrant par l'analyse des issues de 'A[ qui se sont avérées particulièrement saillantes, fréquentes et significatives pour les scriptae à l'étude (chap. 3-7).

(2) Toujours à partir de ce corpus, nous avons entrepris, avec Dumitru Kihaï, une étude lexicologique qui vise à identifier les lexèmes potentiellement francoprovençaux par comparaison avec les lexèmes oïliques en contexte documentaire. À partir de la lemmatisation des formes lexicales, actuellement en cours, nous entendons constituer un glossaire électronique des différents documents notons que les corpus des Documents linguistiques édités sous la direction de Mgr Gardette (Gonon 1974 et Durdilly 1975) n'ont jamais fait l'objet d'un glossaire après la disparition prématurée en 1973 de ce scientifique clairvoyant.

Dans la deuxième étape de ce volet lexicologique nous distinguerons (i) les lexèmes francoprovençaux francisés (ii) des lexèmes partagés par le francoprovençal et le français (éventuellement régional) et encore (iii) des lexèmes oïliques qui ne sont pas partagés par le francoprovençal. Le deuxième type est le plus attendu, si l'on se base sur les habitudes scriptologiques que nous avons pu observer pour le lexique en domaine d'oïl : ${ }^{25}$ les scribes favorisent dans leur sélection des lexèmes à large diffusion, qui ne sont géolinguistiquement pas marqués. Le premier type, les lexèmes francoprovençaux francisés, reste assez fréquent, alors que le troisième, les lexèmes oïliques sans correspondant en

25 Cf. Glessgen (2016; 2017); Glessgen/Videsott (2017). 
francoprovençal, est certainement l'exception, puisqu'il réduit la compréhensibilité et donc la fonctionnalité des textes. Il est toutefois fréquent que des lexèmes indistinctement francoprovençaux et français paraissent sous une forme graphophonétique oïlique.

La contrastivité au sein d'un même ensemble de genres textuels couvrant le tiers nord-est de la Galloromania fait en effet ressortir le vocabulaire francoprovençal de manière assez immédiate. Étant donné que la comparaison portera sur environ 2000 mots, la différenciation tripartite envisagée donnera une idée nette et tout-à-fait nouvelle des interférences linguistiques en jeu au niveau lexical.

Enfin, l'étude individuelle des lexèmes francoprovençaux cernés - la troisième étape de l'étude lexicologique - permettra une analyse de ce vocabulaire en termes scriptologiques, différentiels et évolutifs qui se placera dans le cadre plus général de nos travaux sur le lexique documentaire de la Galloromania médiévale. ${ }^{26}$

Étant donné que la constitution du corpus et l'analyse scriptologique précèdent l'analyse lexicologique, à peine entamée, nous avons exclu cette dernière de la présente étude qui entend identifier le cadre général dans lequel se place la scripturalité francoprovençale.

\section{Le corpus francoprovençal}

\subsection{L’état éditorial}

Le nombre de textes vernaculaires pleins produits en domaine francoprovençal au Moyen Âge est considérablement plus limité qu'en domaine d'oc et d'oïl, en italien ou en espagnol. D’emblée, le recensement peut se concentrer sur le seul ensemble des genres documentaires. Les textes littéraires profanes et religieux sont très peu nombreux - l'aperçu récent de Maffei Boillat peut être considéré comme exhaustif (cf. supra n. 6) - et tous les autres genres, d'un savoir spécialisé (médecine, historiographie etc.), sont absents, en dehors du traité juridique de la Somme du code (cf. infra 8.2).

26 Cf. Glessgen/Tittel (en préparation) pour le vocabulaire oïlique des Documents linguistiques intégré dans le DEAF électronique et Glessgen/Tittel (2018) pour le DAGél dont le système de rédaction dédouble celui du DEAF. Nous préparons actuellement un deuxième dédoublement de ce système permettant de traiter le vocabulaire francoprovençal des Documents linguistique dans la même logique (ib., 817). - Cf. aussi, d'un point de vue méthodologique, Carles/Glessgen (en préparation) pour le vocabulaire régional de l'Est et du Sud-Est oïlique et Carles (2017) pour la catégorisation langagière des lexèmes francoprovençaux en contexte latin. 
Il est par conséquent possible d'avoir une idée relativement précise du volume textuel disponible, d'autant plus que la recherche a poursuivi cette question avec une grande attention. Déjà depuis le milieu du $19^{\mathrm{e}}$ siècle, de nombreux érudits ont entrepris l'inventaire, puis l'édition de documents pertinents, à commencer par des historiens suisses, intéressés autant par les textes latins que vernaculaires :

\begin{abstract}
Recueil diplomatique du canton de Fribourg (Werro/Berchtold/Gremaud 1839-1877 [cf. Müller 1990]), Monuments de l'histoire de Neuchâtel (Matile 1844-1848), Monuments de l'histoire de l'ancien évêché de Bâle (Trouillat/Vautrey 1852-1867), Chartes inédites relatives à la ville et au diocèse de Genève (Mallet 1862); cf. pour la France: Cartulaire de la Ville de Lyon (Villeneuve/Guigue 1876) ou, un peu plus tard, Chartes du Forez antérieures au XIV siècle (Guichard et al. 1933-1980).
\end{abstract}

La tradition philologique ciblée sur les textes romans s'est instaurée vers la fin du $19^{\mathrm{e}}$ siècle surtout en France, quand notamment Édouard Philipon à Lyon, Mgr Devaux à Grenoble ou Jules Gauthier à Besançon ont réalisé un travail de repérage systématique dans leurs régions respectives qui a abouti à des éditions dont la fiabilité répond encore aujourd'hui aux attentes de la critique textuelle :

Philipon : Une page de l'histoire de Lyon [...] Le règlement fiscal de 1351 (1883), Les bénéfices du Chapitre de Saint-Jean ... d'après un terrier en dialect lyonnais du XIIIe siècle (1885), Le dialecte bressan aux XIII ${ }^{\mathrm{e}}$ et XIV $V^{\mathrm{e}}$ siècles (1887), puis surtout Documents linguistiques $d u$ département de l'Ain (1909); cf. aussi Phonétique lyonnaise au XIV siècle (1884), Morphologie du dialecte lyonnais aux XIII ${ }^{\mathrm{e}}$ et XIV ${ }^{\mathrm{e}}$ siècles (1901);

Devaux: Essai sur la langue vulgaire du Dauphiné septentrional au Moyen Âge (1892), Comptes consulaires de Grenoble en langue vulgaire (Devaux/Ronjat 1912);

Gauthier: Les documents franc-comtois des archives de Neuchâtel (1880), Recueil de quarante-huit chartes françaises de 1227 à 1280 pour servir à l'étude du dialecte franc-comtois (1900).

La tradition des repérages et éditions de documents oïliques, francoprovençaux et francoprovençalisants s'est maintenue tout au long du $20^{\mathrm{e}}$ siècle. ${ }^{27}$ Elle a

27 Quelques exemples : Maître Pierre Cudrifin, horloger, et la ville de Romans (Meyer 1892), Un document inédit du français dialectal de Fribourg au XIV siècle (Jeanjaquet 1905), Chrestomathie franco-provençale (Aebischer 1950), L'inventaire des biens d'un citoyen de Lyon en 1327 (Gonon 1966), Extraits du livre de comptes de J. Panczus, receveur de tailles à Lyon (Durdilly 1966), Considérations sur la scripta "para-francoprovençale» (Gossen 1970), Gippons et chapirons ... (Lescuyer-Mondésert 1983), Un journal de recette du péage de Belleville au XV siècle (Horiot/Du Pouget 1990), Une charte jurassienne inédite du début du XIV siècle (Marzys 1994), Latin et langue vulgaire dans les chartes de Bellelay au XIV ${ }^{e}$ siècle (Marzys/Scheurer 1997), L'ordonnance fribourgeoise de 1363 sur les moulins (Amman-Doubliez/Müller 2003), "La Première Collection des lois » 
culminé en 1974 avec plusieurs entreprises de recensement et d'édition tant françaises que suisses qui se sont placées dans le sillage des Documents linguistiques instaurés par Paul Meyer et réalisés pour le département de l'Ain par Édouard Philipon (1909, cf. Glessgen 2015) :

Documents linguistiques du Forez (1260-1498) (Gonon 1974)

Documents linguistiques du Lyonnais (1225-1425) (Durdilly 1975)

Anciens documents en langue française ... cantons de Vaud et de Genève (Gavillet 2011)

Documents linguistiques de la Suisse romande II ... canton de Neuchâtel (Scheurer/Morerod/ Kristol, à paraître)

Documents linguistiques de Fribourg (Fuhrer en préparation) ${ }^{28}$

Ces ouvrages à vocation holistique couvrent en effet une grande partie de la production vernaculaire en domaine francoprovençal. Les séries suisses comportent de manière indistincte les différents documents vernaculaires, indépendamment de la présence ou non du francoprovençal, et visent une certaine exhaustivité au moins pour les $13^{\mathrm{e}}$ et $14^{\mathrm{e}}$ siècles. Les deux publications pour Lyon et le Forez sont plus sélectives et celle pour Lyon exclut les quelques documents oïliques qui commencent à affleurer au même moment que les documents francoprovençaux. ${ }^{29}$ Elles intègrent néanmoins l'essentiel des publications antérieures. Voici un relevé détaillé des éditions disponibles pour le Lyonnais pour l'époque concernée (1 ${ }^{\mathrm{er}}$ q. $13^{\mathrm{e}}$ s. -1476$):^{30}$

de Fribourg en Nuithonie (Ammann-Doubliez 2009), Un censier vaudois du XIV ${ }^{e}$ siècle en langue vulgaire (Maffei Boillat 2016).

28 S'ajoutent pour le territoire d'oïl limitrophe les Documents linguistiques de la Suisse romande I ... Jura et Berne (Schüle/Scheurer/Marzys 2002), provenant pour l'essentiel de Porrentruy. En revanche, la thèse de l'École des Chartes de Philippe Marguerat Documents linguistiques de la Suisse romande (XIII $-X V^{e}$ s.) (1960) est restée sous un format dactylographié et n'a pas connu de diffusion. Il est vraisemblable que les textes édités alors par Marguerat aient également trouvé leur place dans les éditions suisses récentes.

29 D’après l'inventaire des documents francoprovençaux et francoprovençalisants réuni par Durdilly (1975, XXIII-IL), on peut évaluer que son édition comporte au moins un tiers des textes antérieurs à 1400 et conservés aujourd'hui ; cf. aussi Gonon (1974, VIII).

30 Nous renonçons ici à la reproduction des indications bibliographiques des travaux anciens présents dans le tableau puisqu'elles sont intégralement répertoriées par Durdilly. Nous remercions Lorraine Fuhrer de la préparation de ce relevé. 


\begin{tabular}{|c|c|c|c|}
\hline genres textuels & nombre & dates extrêmes & éditions \\
\hline $\begin{array}{l}\text { Tarifs de péages, } \\
\text { leydes, livres de } \\
\text { recettes }\end{array}$ & 15 & $\begin{array}{l}1225 \\
{[c p .1375]-1401}\end{array}$ & $\begin{array}{l}\text { Guigue (1876), Guigue (1882), Philipon } \\
\text { (1884), Aebischer (1950), Perrat/Gardette } \\
\text { (1961), Gardette (1963) : Durdilly (1975) } \\
\text { [= reprend l'intégralité des documents] }\end{array}$ \\
\hline Terriers & 7 & $1^{\text {er }} \mathrm{q} \cdot 13^{\mathrm{e}}-1425$ & $\begin{array}{l}\text { Philipon }(1884,1885 \text { et } 1893), \text { Perroy (1937): } \\
\text { Durdilly }(1975)[=i d .]\end{array}$ \\
\hline Livre de raison & 1 & $1316-1344$ & Durdilly (1975) \\
\hline Livre de comptes & 1 & $1320-1324$ & Guigue/Meyer (1906) : Durdilly (1964 et 1975) \\
\hline Rôle d'arrérages & 1 & 1342 & Philipon (1883) : Durdilly (1975) \\
\hline Comptabilité & 26 & $1343-1476$ & $\begin{array}{l}\text { Guigue M. (1875), Vachez (1877 et 1879), } \\
\text { Philipon (1883, } 1884 \text { et 1905), Guigue (1886), } \\
\text { Durdilly (1966) : Durdilly (1975) } \\
\text { s'ajoutent Durdilly (1981), Bérier (1969), } \\
\text { Salmon (1978), Horiot/Du Pouget (1990) }\end{array}$ \\
\hline Règlement fiscal & 1 & $1351-1352$ & Durdilly (1975) \\
\hline Procès-verbal & 1 & 1355 & $\begin{array}{l}\text { Guigue M. C. (1876), Aebischer (1950), Poncet } \\
\text { (1969) : Durdilly (1975) }\end{array}$ \\
\hline Conventions & 2 & 1359 & Philipon (1884) : Durdilly (1975) \\
\hline Testaments & 2 & $1361-$ fin $14^{e}$ & Guigue (1906), Gonon (1968) : Durdilly (1975) \\
\hline $\begin{array}{l}\text { Estimation de } \\
\text { biens }\end{array}$ & 1 & 1387 & Durdilly (1975) \\
\hline $\begin{array}{l}\text { Livre du vaillant } \\
\text { des habitants de } \\
\text { Lyon }\end{array}$ & 1 & 1388 & Philipon/Perrat (1927) \\
\hline
\end{tabular}

Il en ressort qu'en dehors du Livre du vaillant (1388) et de quelques documents comptables, la quasi-totalité des documents publiés avant 1975 a été rééditée dans le volume de Durdilly.

En prenant en considération les relevés et éditions actuellement disponibles, il en ressort une bonne densité documentaire pour les différentes régions du francoprovençal autant en France qu'en Suisse. ${ }^{31}$ Comme nous l'avons déjà dit, le

31 Nous remercions nouvellement Lorraine Fuhrer d'avoir réuni un premier relevé très vaste, qui a pu prendre appui sur l'état des lieux fourni par Gardette pour le Dauphiné, le Forez et le Lyonnais (1962, 1963 et 1968), les indications fournies par Marguerat (1971), Schüle (1978), Marzys (1978), Gonon (1985), Zufferey (1997) ainsi que l’inventaire raisonné de Vurpas (1995). Il a également pu se valoir des relevés minutieux de Laure Grüner (ms.) et d'Elisabeth Berchtold (ms.). Le répertoire détaillé des éditions de textes documentaires organisé par régions et par genres textuels fera partie intégrante de la thèse en cours de Lorraine Fuhrer. 
Val d'Aoste reste en dehors du domaine d'études - tout comme le Valais et la Savoie - puisque leur scripta documentaire est exclusivement à base latine.

On peut retenir que les premiers textes vernaculaires apparaissent à partir du début $\mathrm{du} 13^{\mathrm{e}}$ siècle mais restent rares, alors que le $14^{\mathrm{e}}$ siècle est plus largement représenté dans toutes les régions. Du point de vue des genres textuels concernés, au sein du territoire français se trouvent presque exclusivement des documents de gestion et de comptabilité en scripta francoprovençale, alors qu'en Suisse les chartes sont fréquentes, toutefois souvent sous une forme fortement oïlique. Les testaments restent rares, comme partout ailleurs dans la Galloromania.

Il va sans dire que les éditions disponibles et les relevés ne couvrent qu'une partie du volume textuel documentaire existant dans les archives du domaine francoprovençal, mais comme nous l'avons dit ces dernières sont malgré tout bien moins riches que les archives des domaines d'oïl et d'oc et - comme Vurpas (1995) l'a souligné - la tradition de la recherche a consacré une énergie considérable dans l'identification des écrits vernaculaires. Les textes publiés garantissent donc un accès tout à fait représentatif aux textes médiévaux selon les trois paramètres de l'espace, du temps et des genres textuels. En mettant en relation le nombre de documents dont on peut supposer l'existence et celui de ceux qui ont été repérés et édités, il est même vraisemblable que le territoire francoprovençal est actuellement un des domaines de la Romania avec la meilleure couverture éditoriale pour les $13^{\mathrm{e}} / 14^{\mathrm{e}}$ siècles.

\subsection{Le corpus francoprovençal numérisé}

Après avoir pris connaissance de la tradition textuelle et de l'état éditorial, nous avons décidé de numériser avant tout l'intégralité des éditions qui se placent dans la tradition des Documents linguistiques, instaurée par Paul Meyer: Philipon (1909), Gonon (1974), Durdilly (1975), Gavillet (2011), Scheurer/Morerod/Kristol (à paraître) et Fuhrer (en préparation). Nous avons ajouté les documents oïliques conservés essentiellement dans le canton du Jura (Schüle/Scheurer/Marzys 2002) qui se placent dans la même tradition et qui sont géographiquement proches. Nous avons ajouté par ailleurs pour le Dauphiné les deux ensembles textuels les plus importants, la Somme du code (Royer/Thomas 1929), traduit et copié à la première moitié du $13^{\mathrm{e}}$ siècle à Grenoble (cf. infra 8.2) et les Comptes consulaires de Grenoble de 1338-1339 (Devaux/Ronjat 1912²).

32 L'édition, préparée par Mgr Devaux a été publiée à titre posthume par Jules Ronjat qui a vérifié la transcription et enrichi l'édition par une analyse linguistique et un glossaire remarquables. 
Ces éditions garantissent une grande fiabilité philologique, essentielle pour l'analyse scriptologique, et elles réunissent un volume textuel important dans un nombre restreint d'éditions, ce qui facilite l'évaluation et la saisie. Numériser une quantité comparable de textes anciens à partir de plusieurs dizaines d'éditions disparates aurait demandé un travail de révision intense, avec un rendement complémentaire bien plus faible. ${ }^{33}$

Nous avons ainsi constitué un corpus de textes documentaires qui couvre toutes les régions du domaine francoprovençal en France et en Suisse qui connaissent des textes vernaculaires pleins à la période médiévale. C'est à contrecœur que nous avons renoncé dans ce cadre à l'étude des textes latins rédigés en territoire francoprovençal et dont l'emploi est quasi-exclusif en Savoie, en HauteSavoie, dans le Valais et en Italie (Vurpas 1995; Gasca Queirazza 2004). Leur traitement demande une méthodologie radicalement différente qui ne peut s'inscrire dans la logique de la linguistique de corpus que nous avons retenue ici.

Dans le cadre des Documents linguistiques, ces éditions rendent possible une analyse contrastive du domaine francoprovençal et du domaine oïlique, en vue d'établir avec sécurité les éléments qui sont propres voire exclusifs à la scripta francoprovençalisante. ${ }^{34}$ Le corpus émanant du territoire francoprovençal est désormais implanté et interrogeable sur le site des DocLing. Il comprend un ensemble d'environ 330 documents, de longueur très variable, s'échelonnant entre 1225 et 1498. La liste suivante précise l'étendue géo-chronologique du corpus numérisé (dans l'ordre chronologique d'abord pour la France, puis pour la Suisse) :

Dauphiné : 2 documents volumineux (déb. $13^{\mathrm{e}} \mathrm{s}$. [cp. $1^{\mathrm{re}} \mathrm{m} .13^{\mathrm{e}} \mathrm{s}$.] ; 1338/1339) : la Somme du code [Royer/Thomas 1929] et les comptes consulaires de Grenoble (en deux parties) [= Devaux/Ronjat 1912]

Lyonnais : 49 documents ([1225]-1425) : 1 livre de raison, 1 fragment de livre de comptes, 2 testaments, 6 pièces administratives, 7 terriers, 15 tarifs de péages, leydes, livres de recettes, 17 comptes [= Durdilly 1975]

Cette publication se place ainsi dans la même lignée et répond aux mêmes exigences que le travail de Paul Meyer et d'Edouard Philipon.

33 Un apport de poids sera en revanche l'édition de des comptes de la seigneurie de ThoireVillars (originaire du Bugey, Ain) dont le manuscrit avait été préparé par Jeanne-Marie LescuyerMondésert (cf. 1983). La publication révisée et élargie de ce corpus volumineux du troisième quart du $14^{\mathrm{e}}$ siècle (1361-1371) est poursuivie par Claudine Fréchet (2004).

34 La numérisation et la mise en ligne des Documents provençaux et dauphinois - dans sa part occitane - de Meyer (1909), toujours dans le cadre des DocLing électroniques, permettra dans cette même optique de préciser les modalités de transition entre le francoprovençal et l'occitan provençal et d'affiner encore la nature de la scripta francoprovençale en la replaçant dans le réseau scriptologique galloroman qui était le sien au Moyen Âge. 
Forez : 63 documents (1260-1498) : 6 chartes, 1 testament, 1 liste de vassaux, 4 inventaires, 1 fragment de mémoriaux, 1 extrait de minutes notariales, 2 terriers, 13 registres audienciers bilingues, 34 comptes ou registres de comptes [= Gonon 1974]

Ain : 18 documents $(1265-1365 ; 1465 / 1466): 7$ chartes, 1 lettre, 2 comptes, 8 terriers [= Philipon 1909]

Neuchâtel : 56 documents (1238-1379), essentiellement des chartes [= Scheurer/Morerod/ Kristol, à paraître $]^{35}$

Genève, Lausanne et pays de Vaud: 72 documents (1247-1406) : 60 chartes, 6 lettres, 5 comptes, 1 censier [ = Gavillet 2011]

Fribourg : 74 documents (1293-1496) : 59 chartes, 2 lettres, 6 comptes, 7 extraits de livres de dettes [= Fuhrer, en préparation]

La richesse de ce corpus de près de 770000 mots réside, au delà de la qualité philologique des éditions, dans la pluralité des genres représentés - au moins parmi les genres documentaires -, dans l'étendue géographique et chronologique des textes et aussi dans le fait que chaque document est daté et localisé (avec plus ou moins de précision), ce qui permet de l'insérer de manière sûre au sein du diasystème.

\subsection{Les corpus des DocLing des territoires oïliques et francoprovençaux}

Une fois numérisé le corpus francoprovençal, nous avons entrepris les premiers sondages d'analyse scriptologique et lexicale. Nous nous sommes alors rapidement rendus compte qu'il était indispensable d'examiner par contrastivité une partie significative des scriptae du domaine d'oïl. Or, la base de données des DocLing couvre toutes les régions oïliques pertinentes pour une comparaison : la quasi-intégralité des régions du sud-est oïlique, la Franche-Comté et la Bourgogne ainsi que la Lorraine et la Champagne, puis Paris avec la Chancellerie royale, et - en contraste - la Picardie qui a développé en premier une scripta oïlique continentale (cf. Glessgen 2017). ${ }^{36}$ En revanche, les régions de l'Ouest ainsi que la Wallonie - dont l'absence dans les DocLing est très regret-

35 Dans la collection de Scheurer/Morerod/Kristol, il s'agit des documents $\mathrm{n}^{\circ}$ 3, 5, 7, 9-12, 18 , 22s., 25, 33s., 37, 39, 42, 50, 56, 60, 63, 65, 68, 71s., 142, 145s., 151, 154-157, 159-182. L'édition comporte par ailleurs 126 actes qui semblent avoir été rédigés essentiellement à Besançon (Doubs) et que nous avons également intégrés dans les Documents linguistiques électroniques.

36 Dans la couverture géographique ne manquent actuellement que la collection de la Moselle ainsi que celle du Doubs qui seront toutefois intégrées au courant de 2019. 
table pour d'autres interrogations - ne seraient pas d'une grande utilité dans notre contexte.

La comparaison entre les documents francoprovençaux et le tissu scriptologique de la langue d'oïl entre la Picardie et la Franche-Comté s'est en effet avérée très parlante alors que quand nous nous étions concentrés, au début, sur les seules séries provenant du territoire francoprovençal et des départements immédiatement voisins nous n'arrivions pas à établir une structuration géolinguistique et scriptologique cohérente.

Pour faciliter l'argumentation par la suite, nous antéposons nos conclusions concernant la structure géo-scriptologique macroscopique du territoire à l'étude. Il faut en effet distinguer six ensembles; nous considérons avec une attention particulière les deux volets francoprovençaux, déjà identifiés auparavant :

(i) le territoire francoprovençal de la France actuelle, à savoir les séries du Lyonnais, de l'Ain au Nord, du Forez à l'Ouest et du Dauphiné - plus précisément Grenoble - au Sud-Est

(ii) le territoire francoprovençal de la Suisse actuelle : les séries de Fribourg, de Neuchâtel et de Vaud/Lausanne/Genève

Interviennent ensuite les quatre ensembles oïliques dont chacun apporte des éléments complémentaires à l'interprétation :

(iii) le domaine sud-oriental voisin du francoprovençal : la Franche-Comté (Haute-Saône, Jura [FR] et Doubs) et le canton du Jura ${ }^{37}$ ainsi que la Bourgogne (Côte-d'Or, Nièvre et Saône-et-Loire) $)^{38}$

(iv) le domaine oriental : la Lorraine (Meurthe-et-Moselle, Meuse et Vosges) et la Champagne (Marne et Haute-Marne)

(v) Paris (représenté par la chancellerie royale) ${ }^{39}$

(vi) la Picardie (représentée par les archives de la ville de Douai)

Il est évident que la distribution chronologique de notre corpus est tributaire de plusieurs paramètres difficiles à maîtriser tels que les aléas de la production

37 Rappelons que les documents conservés dans le canton du Jura ont été presque tous rédigés à Porrentruy (cf. supra n. 28).

38 Dans l'interprétation, nous regrouperons, quand c'est possible, dans les tableaux les séries de ces cinq départements, de même que les cinq suivants pour la Lorraine et la Champagne.

39 Nous avons pris en considération les 120 documents originaux sans les 20 copies, mais avec les 8 documents picardisants. Parmi ces derniers ont été rédigés à Paris : RP 129703 20 01, RP 1297 0320 02, RP 12970332 01, RP 12970332 02; ont été rédigés en Picardie : VRP 12970530 01, RP 12970612 02, R 12970612 05, RP 12970612 08, RP 12970612 08, RP 1297082901. 
textuelle au Moyen Âge, l'état des éditions modernes ou encore les choix du projet centenaire des DocLing. Néanmoins, le corpus tel qu'il se présente répond à l'idée fondamentale du projet qui est de documenter de manière idéalement exhaustive les débuts de l'écrit vernaculaire. Or, ces débuts se placent dans la première moitié du $13^{\mathrm{e}}$ siècle pour la plupart des territoires d'oïl et dans le dernier quart du $13^{\mathrm{e}}$ siècle pour la chancellerie royale et le domaine francoprovençal (France et Suisse).

La Picardie se voit représentée par un ensemble exceptionnel d'environ 500 documents provenant d'un même lieu - Douai - et couvrant intégralement la première époque de l'écrit documentaire français, soit entre 1204 et 1270. Les autres séries oïliques orientales des DocLing se placent surtout entre 1230 et 1270, les documents sud-orientaux, quant à eux, entre 1250 et 1330. Les documents du domaine francoprovençal font également apparition au milieu du $13^{\mathrm{e}}$ siècle, mais se placent essentiellement entre la fin du $13^{\mathrm{e}}$ et la fin du $14^{\mathrm{e}}$ siècle. Ces décalages chronologiques incontournables n'empêchent pas la comparaison des données, mais sont à prendre en considération à tout moment. Voici l'aperçu chronologique global qui est organisé d'après les six ensembles géo-scriptologiques retenus et dans l'ordre des tableaux suivants $:^{40}$

\begin{tabular}{lcc}
\hline lieu & dates extrêmes & $\begin{array}{c}\text { nombre de doc. dans les } \\
\text { DocLing }\end{array}$ \\
\hline Dauphiné & domaine francoprovençal & \\
\hline Lyonnais & $1^{\text {re }} \mathrm{m} .13^{\mathrm{e}} \mathrm{s.} ; 1338 / 1339$ & $\begin{array}{c}\text { [Somme du code } \text { comptes } \\
\text { consulaires Grenoble }]\end{array}$ \\
\hline Ain & {$[$ ca 1300$]-1425$} & 49 \\
\hline Forez & $1265-1466$ & 18 \\
\hline Fribourg & {$[1270]-1498$} & 63 \\
\hline Vaud, Lausanne, Genève & $1293-1496$ & 74 \\
\hline Neuchâtel & {$[$ ca 1280$]-1406$} & 72 \\
\hline & {$[$ ca 1265$]-1379$} & 56 \\
\hline
\end{tabular}

40 Nous avons indiqué entre crochets le moment où s'instaure une documentation suivie, en faisant abstraction de quelques actes antérieurs; par ailleurs, nous avons distingué dans nos relevés les documents de la collection de Neuchâtel qui proviennent de Besançon « Doubs [Nch] » de ceux qui ont vraisemblablement été rédigés à Neuchâtel (« Neuchâtel »). Cf. Glessgen (2016; 2018) ainsi que le site des DocLing. 


\begin{tabular}{lcc}
\hline \multicolumn{1}{c}{ lieu } & dates extrêmes & $\begin{array}{c}\text { nombre de doc. dans les } \\
\text { DocLing }\end{array}$ \\
\hline Franche-Comté, Bourgogne & domaine d'oïl & 592 \\
\hline Doubs [NCh], Jura [CH] & $1227-1331$ & 425 \\
\hline Champagne, Lorraine & {$[$ ca 1265$]-1395$} & 1179 \\
\hline Douai & $1225-1275$ & 506 \\
\hline Chancellerie royale & $1204-1270$ & 140 \\
\hline & {$[1280]-1300$} & 2842 actes \\
& & 1250000 mots \\
\hline
\end{tabular}

La tradition des actes retenus est, quant à elle, idéale dans la mesure où le corpus est intégralement constitué de documents originaux ${ }^{41}$ et d'éditions diplomatiques. Aussi, il s'agit de la plus importante collection galloromane philologiquement fiable qui ait jamais été étudiée de manière quantitative à des fins graphophonétiques et morphologiques. Les documents du domaine francoprovençal sont d'ailleurs en moyenne bien plus volumineux que ceux du domaine oïlique, puisqu'il s'agit souvent de registres et non pas de chartes individuelles; ils réunissent environ 770000 mots contre 1,25 million pour le domaine d'oïl, soit plus d'un tiers du corpus. Si la structuration à la fois géolinguistique et chronologique des données s'avère lourde dans sa mise en œuvre, une base aussi large et fiable présente l'avantage de réduire au maximum la contingence des résultats obtenus. ${ }^{42}$

\section{3 Étude scriptologique des documents francoprovençaux}

\subsection{Les interrogations scriptologiques}

L'approche scriptologique a comme objectif premier de distinguer les différents niveaux langagiers qui sont coprésents dans les textes rédigés en domaine francoprovençal. Nous nous plaçons dans le cadre théorique et méthodologique

41 Ou de copies contemporaines à moins de vingt ans de distance.

42 Citons dans le même ordre d'idées, Gossen $(1968,4)$ : «plus le nombre de pièces analysées sera grand, plus grande sera la probabilité que nos résultats seront valables ». 
établi par les études de Remacle (1948), Gossen (1964-1970), Goebl (1975a/b; 1979), Dees (1980; 1985), Pfister et, plus récemment, Völker (2003), Videsott (2013; 2015a/b) ou Grübl (2013; 2014; 2015), cadre auquel nous avons également essayé de contribuer à travers ces dernières années. ${ }^{43}$

Le point de départ empirique de notre analyse scriptologique est, par la force des choses, l'identification des paramètres à étudier. Cette opération représente une recherche à part entière qui doit prendre en considération autant le savoir existant sur les scriptae médiévales que les données qui émergent à travers les analyses et qui est tributaire des modalités d'une interrogation quantificatrice et semi-automatisée. ${ }^{44}$

Nous avons ainsi réuni dans un premier temps un ensemble de paramètres grapho-phonétiques et morphologiques qui opposent le francoprovençal ancien au français. Grâce à l'excellente tradition de la recherche, ce premier pas d'orientation repose sur une base solide. Pour les éléments phonétiques et morphologiques, déjà Hafner (1955) et Horiot (1972) fournissent un cadre global assez complet ; pour la morphologie verbale s'ajoute, plus récemment, la synthèse de Maffei Boillat (2015, 91-100 ; cf. aussi Philipon 1901) ; retenons par exemple :

- $\quad$ dans le vocalisme accentué les évolutions de 'a[ non précédé de palatale (afrpr. pra $/$ afr. pré < 'PRATU) ou suivi de nasale (afrpr. mann / afr. main < 'MANU), celle de 'ū devant nasale (afrpr. aucon / afr. aucun < ALI'cunU), celle de 'è[ (afrpr. seir / afr. soir < 'SERU), celle de 'è[ devant nasale (afrpr. fein / afr. foin < lat. 'FENU) ou encore celle de 'ŏ + vélaire (afrpr. lue / afr. lieu < 'Locu) [Hafner 1955, 16-22, 30-36, 63-71, 75s., 79-81, 112-114] ;

- dans le vocalisme inaccentué le maintien du timbre des voyelles finales (afrpr. terra / afr. terre < TERRA, afrpr. favro / afr. fèvr $\underline{e}$ < FABRU) ainsi que l'évolution de -a après palatale (afrpr. vigni / afr. vigne < vinEA) [ib., 126-134] ;

- $\quad$ en morphologie et syntaxe, le type afrpr. li pour l'article défini fém. sg. cas sujet (afr. la) et afrpr. lo $(u)$ au masc. sg régime (afr. le mais parallèle à afr. rég. lo) et, surtout, mais plus difficile à cerner informatiquement, le maintien de la flexion à deux cas jusqu'au début du $16^{\mathrm{e}}$ siècle [Horiot 1972, 43] ;

- $\quad$ en morphologie verbale, la désinence -ont dans la $3^{\mathrm{e}}$ pers. pl. ind. prés. (afr. -ent) [Maffei Boillat 2015, 100].

Mais déjà un premier aperçu à partir des balisages que nous avons entrepris pour les Documents linguistiques en partant des paramètres pertinents pour les scriptae lorraines nous a amenés à quelques ouvertures. Ainsi, nous avons pu constater

43 Les titres de référence sont réunis dans la bibliographie (chap. 9).

44 Cf. nos réflexions à ce sujet dans la préparation de l'analyse des documents de la Meurthe-etMoselle, Glessgen (2008, 446-448). Nous avons retenu alors pour guider nos choix la pertinence des variables en question, leur fréquence et la facilité de son identification en vue du balisage informatique. 
que les graphèmes 〈k〉 et 〈w〉, qui sont assez présents dans les scriptae oïliques nord-orientales et sud-orientales, sont très rares dans les textes du domaine francoprovençal, ce qui constitue une opposition ex negativo entre les deux territoires.

Nous avons également analysé de manière traditionnelle un certain nombre de documents de notre corpus francoprovençalisant pour avoir une idée plus précise des manifestations concrètes qui peuvent être relevées à l'aide de nos outils de balisage. Nous présenterons par la suite trois brefs documents, qui permettent en même temps de se faire une idée de la physionomie des scriptae mixtes à l'étude.

\subsection{Exemples de textes}

Nous avons retenu ici deux chartes rédigées en Suisse romande, l'une éditée dans la collection de Neuchâtel de Rémy Scheurer, Jean-Daniel Morerod et Andres Kristol (à paraître), l'autre par Lorraine Fuhrer dans le cadre de son doctorat. Ces deux actes sont intégrés à la plateforme zurichoise AdFontes, qui fournit un outil pédagogique très utile pour l'apprentissage paléographique et éditorial. ${ }^{45}$ Nous prendrons ensuite à titre d'exemple un document de l'Ain, édité par Édouard Philipon (1909), qui fait donc partie du monument fondateur des Documents linguistiques.

\subsubsection{La charte de Neuchâtel de 1265}

Cette brève charte de 1265 représente l'un des premiers textes vernaculaires pleins réalisés à Neuchâtel. Les Archives de l'État de Neuchâtel conservent certes des témoins plus anciens mais rédigés à Besançon, en domaine d'oïl. Le document en question est un acte privé, un acensement, qui fait intervenir plusieurs acteurs laïques qui n'appartiennent pas à la noblesse ; le bénéficiaire de l'acte est un bourgeois de la ville. En voici le texte, édité d'après les critères des Documents linguistiques électroniques : ${ }^{46}$

1 Sachont tuit cil qui veront ces presentes lestres 2 que je, Girar, fiz Aubertin $\backslash 2$ dit de la Tort, donzez de Nuf_chastel, 3 hai dona hà Martin, fiz Donnier, borgés de \3 Nuf_chastel, à lou et à ces hert, à cens port dozez diniers de la monee de esteve/4nens tot lou tinement lou quel

45 Cf. Carles (2008-2016).

46 Cf. DocLing (« Descriptif du projet » > « Critères d’édition »). 
donz Roz de Corfranou tiniert de $\backslash 5$ Aubertin, mon parre, in terra, in praz, in chans, in chesauz, in arbres et in $\backslash 6$ autres choses. 4 Et covent li hai per ma foi donae estre bons werenz de $\backslash 7$ cesta chosa devanz dita encontres toz, lou et hai ces hers. 5 De cesta chosa $\backslash 8$ sont tesmoniages Renauz de Bavenz, Hyremanz dit de Fressen, W. li 19 marchianz et Girardons. 6 Et à la plus grant certanitay de cesta chosa, je, \10 devanz dit Girar, hay dona cest lestres sielaes dou siel a religious ba\11ron et honestou abés de Fontanna Andrer. $7 \mathrm{Ce}$ fu fet en l'ant de l'en_carnation \12 Nostre Seignor que li milliares coret per mil et .CCo. et sexante et quatrou ou \13 meis de juanier.

D'un point de vue de la stratification linguistique, si la part française reste bien présente, les éléments francoprovençaux sont très apparents voire dominants dans certaines parties de l'acte, ce qui est d'ailleurs plutôt l'exception en Suisse romande. Le français détermine notamment le début et la fin de l'acte, la publicatio (1) et la datatio (2), où des indices francoprovençaux restent ponctuels («sachont〉 au lieu de 〈-ent〉; 〈quatrou > ou le type 〈juanier〉). Dans un certain sens, le français cadre ainsi le document, de la même manière que les premiers documents vernaculaires étaient cadrés par des parties textuelles latines. Cette distribution souligne notre idée selon laquelle le francoprovençal s'est glissé dans un moule oillique préexistant.

La présence francoprovençale devient particulièrement apparente dans le vocalisme (cf. la synthèse supra, 4.1) ; on relève ainsi :

- le maintien de A accentué libre non précédé de palatale : práz 1. 5, parre 1. 5, dona (p. p. m.) 1. 2; 9, dona $\underline{a}$ (p. p. adj. f.) 1. 6, certanitay (s.f.) 1.9 sieláes 1.9 ; cf. aussi, avant [1] chesauz 1.5 , forme qui n'est pas complètement inconnue du domaine d'oïl (cf. infra 5.6) ; nous avons expliqué plus loin la nature et la genèse vraisemblable des variantes fém. sg. - ae, -ay et pl. -aes (cf. infra 5.2);

- le maintien de A final inaccentué : terra 1.5 , cest $\underline{a}$ chos $\underline{a} 1.7$ (bis) ; 9, dita 1. 7, Fontanna 1. 11 (l'issue oïlique est moins fréquente avec mone $\underline{\text { }} 1.3$ et sexante 1.12 ; pour le type dona $\underline{e}$ l. 6 cf. encore infra 5.2 ; le pluriel en 〈es〉, enfin, est autant caractéristique du frpr. que du fr. : presentes lestres 1. 1, autres choses 1. 6, lestres 1. 9);

- le maintien de [o] final inaccentué après un groupe consonantique, graphié ici 〈ou : honestou 1. 11, quatrou 1. 12.

Les marques grapho-phonétiques sont donc très saillantes dans ce texte qui se rattache par ailleurs à une scripta oïlique de type sud-oriental (l'article lou l.3, par exemple, est propre autant au frpr. qu'au fr. du sud-est). D'un point de vue lexical en revanche, l'acte ne comporte aucun type qui différencierait le francoprovençal du français. Nous sommes donc pleinement dans le cas de figure des «lexèmes partagés par le francoprovençal et le français», évoque auparavant (chap. 1.4) 


\subsubsection{La charte de Fribourg de 1363}

La seconde charte, de Fribourg, a été rédigée un siècle plus tard, en 1363, vers la fin de l'époque centrale de l'élaboration scripturale du francoprovençal. Voici le texte :

1 Je, Johant Velga, chevalier, advoyez de Fribor et nos, li $\backslash 2$ consed, et la communitey de Fribor $\mathbf{2}$ faczons savoir à totz 3 que, $\backslash 3$ nos, à-grant deliberacion intre nos pluissiours foys ehu, acordablemant $\backslash 4$ avons ordoney, fermemant à tenir, 4 que nulle persone quelque $\backslash 5$ cil soyt qui aura jurez furs de nostre vile ou por enons $\backslash 6$ ou por autre chose, que dix ore avanz un lo repaleyt per $\backslash 7$ requeste que un fist per lettre. 5 Et ce presant statut $\backslash 8$ volons qu'il duroyt tanque de nos soyt repalez acordab\9lemant. 6 In-tesmognyage de ceste chose, nos li advoyez, $\backslash 10$ li consed et la communitey de Fribor, le seel de nostre communitey avons \11 mis en ceste lettre. 7 Doné le derier jor dou moys de decembre, \12 l'an de grace corant mil .ccc. et sexante et troys, sellon le $\backslash 13$ stile de Lausanne.

Les traits francoprovençaux dans l'habillage grapho-phonétique sont moins apparents que dans l'acte précédent ; l'on relève notamment l'absence de «a accentué (communitey 1. 2) et de 〈-a〉 final inaccentué (nulle persone 1. 4, vile 1. 5, chos $\underline{e}$ 1. 6, lettre 1.7 etc.). Ce constat est surprenant, puisque - comme il est connu depuis longtemps et comme nous le verrons aussi plus loin - la scripta de Fribourg est la plus francoprovençalisante parmi les scriptae suisses. Nous observons en effet au plan morphologique le maintien, au moins partiel, de la flexion bicasuelle, qui, dans la seconde moitié du $14^{\mathrm{e}}$ siècle, est clairement attribuable au système linguistique francoprovençal et non oïlique :

- le substantif advoyez s. m. sg. suj. l. 1 avec le graphème 〈z〉 notant l'affriquée produite entre la dentale cachée et le morphème flexionnel du nominatif singulier $\{-\mathrm{s}\}$; le participe passé jurez 1.5 ;

- l'article m. sg. sujet $l i 1.1 ; 9 ; 10$, sachant que le substantif suivant consed, quant à lui, ne porte pas de marque.

La flexion bicasuelle est en revanche absente tout au début de l'intitulatio (Johant Velga, chevalier) et dans la datatio (le derier jor), ce qui rejoint l'observation d'un cadre oïlique que nous avons déjà pu faire pour l'acte de Neuchâtel.

Par ailleurs, cet acte introduit deux lexèmes propres au francoprovençal, l'un par le sens, l'autre pour l'unité lexico-sémantique (forme/sens) :

- le substantif masculin advoyez est bien répandu en domaine d'oïl mais son acception est sans équivoque régionale; il désigne le 'chef d'un gouvernement, premier magistrat', spécialisation sémantique caractéristique de l'administration fribourgeoise et attesté dans notre corpus entre 1293 et 1496 (cf. FEW 24, 203a, s.v. ADvocatus);

- le substantif masculin enon 'amende' est une forme inconnue en domaine d'oïl et également propre aux textes rédigés à Fribourg. Cet emprunt à l'ancien all. de Suisse 
Einung y est attesté entre 1363 (notre texte est légèrement antérieur à la première attestation jusqu'ici connue de 1368) et 1410 (cf. DMF s.v. einon, GPSR s.v. ; dans FEW 23, 129a amende le mot est placé parmi les 'Inconnus').

\subsubsection{Le terrier de Bagé-le-Châtel (Ain)}

Le troisième et dernier exemple retenu est extrait d'un terrier qui concerne la seigneurie de Bâgé-le-Châtel (aujourd'hui commune de l'arr. de Bourg-en Bresse) et qui est daté par une fourchette chronologique comprise entre 1294 et 1323. Le document est conservé en original aux A.D. de la Côte-d'Or (Chambre des Comptes de Bourgogne, B 570) et a été édité par Édouard Philipon (1909, 26s.). Nous avons modifié pour les commodités du commentaire linguistique la mise en page de l'éditeur en introduisant des alinéas et une numérotation au début de chaque nouvelle phrase et en adaptant sur la base de nos résultats d'analyse certains choix éditoriaux (résolutions d'abréviations, accents) :

1 Martins Tavanaz est homz tall. [résolu talliablos] mon seignor, et tient de sey .iiij. meytera(e)s de terra et .j. meytera de bos et lo pra de iiij. charres de fein.

2 Item, la syseyma partie d'une benna en Sonnan. Item, la quarta partie en .ij. seis a peschier, en les Ayies.

3 Item, la syseyma d'une seis en l'ivoiglli a peschier.

4 Item, la syseyma partie de la [pe]cheri d'una riveri vers les Motauz.

5 Per les quaz choses il deit mon seignor la talli, les corva(e)s et la taschi de .ij. meytera (e)s et .iiij. s. $\operatorname{cop} a(e) s$ de la dita terra et .iiij. s.et .xj. d. par. et la quarta partie d'una copa de froment de renta.

6 Et deit chascuns fues .j. gelina.

7 Item, tint del seignor de Monbelet .ij. copes de terra a .vj. d. par. de renta.

8 Item, tint de cel mesmo seignor de Monbelet en atro lue .j. copa de terra a .vj. d. et .j. pogeysi parisis de renta ; et sunt el dit tinement ses maysons. [...]

Nos remarques linguistiques concernant ce texte sont non-exhaustives comme précédemment et reprennent les paramètres réunis supra (3.1). On observe :

- le maintien de 'a accentué libre non précédé de palatale : pra 1 , qua $z 5$ (< QUALE) ; ${ }^{47}$

47 Le s.f. copa sg. / copes pl. est formellement équivoque. Il s'agit soit du continuateur de la forme simple (lat. CUPPA), soit du dérivé (comme mfr. régional coupée) ; «-a〉 final représentant tantôt le maintien de la voyelle finale inaccentuée ou celui de la voyelle accentuée libre. Les deux lexèmes de même sens sont attestés dans la région au Moyen Âge (cf. FEW 2,1554b). Philipon tranche, quant à lui, pour la forme dérivée puisqu'il introduit l'accent aigu sur la forme pl. «copés〉 (7). Il s'agit selon nous de la solution la moins économique, dans la mesure où il faudrait alors 
- le maintien de la voyelle finale inaccentuée au féminin, présent tout au long de

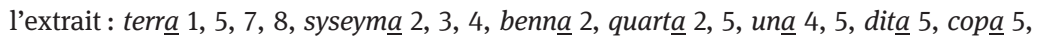
rent $\underline{a} 7,8$. Le traitement oïlique est minoritaire comme dans la charte de Neuchâtel et restreint à un nombre défini de lexèmes (partie 2, 4, 5, une 2, 3). Le maintien de la voyelle inaccentuée se relève également au masculin : mesmo, atro 8 ;

- le traitement particulier de 'a inaccentué précédé de la palatale: ivoiglli s. f. "étang poissonneux' 3, [pe]cheri et riveri 4 ;

- le traitement également particulier de 'ŏ + vélaire : fues 6 (< FOCU);

- le traitement retardé de la diphtongaison de 'ê[ : sey pron. 1, seis s. f. 'emplacement' 2 (cf. FEW 11, 409a, s.v. SĒDES : abress sei, seis ; on notera que les cognats de cet étymon dans ce sens sont exclusivement afrpr. et aocc., cf. FEW ib. sous 1.) ; deit v. 5;

- le traitement également retardé de 'è[ devant nasale : fein s. m. 1 et siseyma adj. f. 2, 3, 4 ;

- la présence la flexion bicasuelle (y inclus sur le sujet anthroponymique): Martins Tavanaz et son attribut hom $\underline{z} 1$, chascuns fues 6 .

\subsubsection{Commentaire}

Ces trois textes n'épuisent bien entendu pas la complexité des interactions et élaborations qui font le propre des scriptae en domaine francoprovençal. Ils suffisent toutefois pour illustrer la coprésence des deux systèmes linguistiques dans les textes et également la notable variabilité dans leur combinaison. Ils suffisent également pour illustrer

(1) que le francoprovençal a une existence scripturale indéniable au Moyen Âge et

(2) que son élaboration n'est jamais totalement indépendante du français qui doit ainsi être considéré à tout moment dans l'étude des scriptae en question.

Ils montrent par ailleurs que le lexique de ces textes est généralement partagé - au delà de son marquage graphématique - par les deux langues, avec tout au plus quelques mots exclusifs au francoprovençal.

Sur la base de ces constats, nous essayerons par la suite de dépasser l'analyse des textes individuels par l'approche quantificatrice de l'analyse scriptologique qui, seule, permet de mettre au jour avec précision la stratification langagière sous-jacente.

expliquer copes comme une forme ne marquant pas «e` du féminin (on attendrait copees en afr. et copaes ou copas en afrpr. ; cf. infra 5.2).

Le cas de figure est récurrent dans nos corpus et le problème se pose encore dans le présent extrait pour charres (1) qui repose, selon nous, plutôt sur CARRU (avec changement de genre) que sur CARRATA. 


\section{Le dialogue entre scriptae francoprovençales et françaises. Interrogations et méthodes d'analyse}

\subsection{Interrogation de base}

Les issues de 'A[ dans les variétés galloromanes comportent une notable complexité en fonction de leur entourage. Elles se caractérisent par ailleurs par une haute fréquence puisqu'elles impliquent, entre autre, les verbes de la première conjugaison (inf. en -ARE, p. passé en -ATU/-ATA). Si la problématique est très présente dans la tradition d'études, elle est loin d'avoir été élucidée dans toute sa complexité. Pour notre interrogation, elle est particulièrement précieuse parce qu'elle permet d'évaluer les choix scriptologiques en territoire francoprovençal sur une base quantitative sûre.

Nous avons distingué dans notre étude scriptologique six sous-ensembles concernant les issues de 'A[, en les distinguant en fonction des phonèmes précédents (radical terminant par une consonne palatale voire $/ \mathrm{i} \sim \mathrm{j} /$ ou non) et suivants ('A[ suivi de nasale ou non, de $/-\lambda /$ ou non, de $/ \mathrm{rj} /$ ou non). Nous traiterons par la suite les cas de ${ }^{\prime} \mathrm{A}$ [ non suivis de nasale ou de $/-\lambda /$, en distinguant ceux qui ne sont pas précédés de palatale de ceux qui se placent après palatale, /i/ ou /j/. De manière très globale, l'on retient pour ces deux ensembles les principales issues suivantes :

\begin{tabular}{|c|c|c|}
\hline lat. ['a]_V & frpr. ['a] $\langle a\rangle$ & ['pra] 〈pra〉 \\
\hline & fr. ['ع: 'e:] 〈e〉 fr. ['ei] 〈ei〉 & 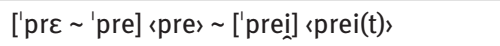 \\
\hline \multirow[t]{2}{*}{ lat. PAL_['a] } & frpr. ['je] 〈ie»> frpr. ['ja] 〈ia〉 & {$\left[\right.$ mar't $\left.^{\prime} f j \varepsilon\right]\langle$ marchie» $\rangle[$ mar'tfja] $\langle\operatorname{march}(\mathrm{i}) \mathrm{a}\rangle$} \\
\hline & fr. $\left[{ }^{\prime} j \varepsilon\right]\langle i e\rangle$ & {$\left[m^{\prime} r t f j \varepsilon\right]\langle m a r c h i e\rangle$} \\
\hline
\end{tabular}

Le premier type, que nous avons déjà vu dans la charte de Neuchâtel et dans le terrier de Bagé-le-Châtel (cf. supra), reflète une opposition phonétique nette entre le français et le francoprovençal. Déjà Ascoli avait relevé le maintien de 'A[ comme l'une des caractéristiques phonétiques saillantes du francoprovençal (cf. Hafner $\left.1955, \S 1^{48}\right)$. La position après palatale est plus délicate parce que, dans un premier

48 Cf. également Devaux (1892, 104-108), Philipon (1884, 542-544; 1887, 13 ; 1893, 4, 7 et 9s. ; 1909, 105, 140 et 153), Gossen (1964, 347), Vurpas (1995, 399), Maffei Boillat $(2015,66)$. 
temps, le francoprovençal connaît la même issue que le français ['jec], avant de s'en distinguer par une nouvelle ouverture vocalique ['ja] (cf. Hafner 1955, § 9 et infra n. 85) ; ici, le phonème /a/ n'est donc pas primaire mais secondaire.

\subsection{Le balisage des issues de 'A[ précédé ou non d'une consonne ou d'un groupe palatal}

Le balisage des occurrences individuelles est réalisé à l'aide du logiciel phoenix-2 qui gère les textes du corpus, la lemmatisation et les analyses graphématiques et morphologiques..$^{49}$ Le logiciel prévoit des interrogations faisant appel aux expressions régulières. Pour relever les issues de ${ }^{A} \mathrm{~A}$, il s'agissait donc de formuler des interrogations portant sur les finales (du type « ei $(\mathrm{s} \mid \mathrm{z})$ ? \$ » pour « «-ei final suivi ou non de $\langle\mathrm{s}\rangle$ ou de $\langle\mathrm{z}\rangle\rangle)$, de vérifier chaque occurrence dans son contexte et d'attribuer une catégorie préétablie aux formes pertinentes (du type "1110" correspondant à « «-ei(s/z)» non précédé de palatale, provenant de lat. -ATU ») : ${ }^{50}$

49 Une version partielle de ce logiciel est accessible à partir du site internet des Documents linguistiques (DocLing): 'Les corpus textuels' > 'Interrogations linguistiques'; ici, les mêmes opérations de recherche sont accessibles et il est également possible d'exporter les résultats dans un fichier excel. Le lexique lemmatisé des DocLing, quant à lui, est intégré désormais pour sa part oillique dans le DEAFél et ces occurrences comportent un hyperlien à l'édition électronique des DocLing. La base de données scriptologique, dans laquelle nous avons réuni les quelque 300000 occurrences actuellement balisées, reste encore interne mais sera mise en ligne dans le cadre du projet FNS en cours.

50 Nous avons naturellement été amenés à créer certaines de ces catégories au cours de l'opération de balisage, tant la variance graphématique est parfois imprévisible. 


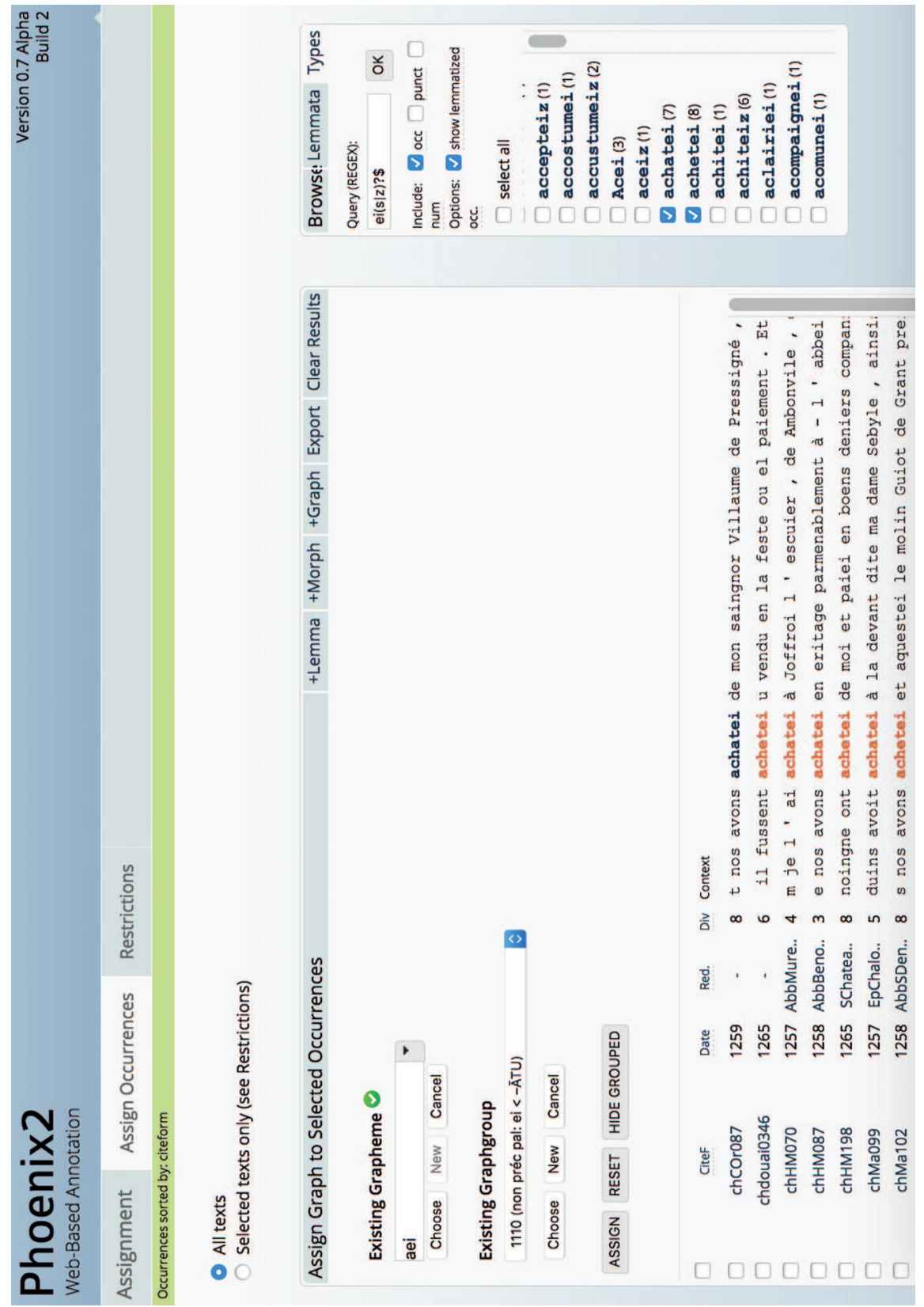

III. 2 : Le relevé de formes grapho-phonétiques définies dans phoenix. 
Les résultats de cette catégorisation sont gérés ensuite dans une interface complémentaire, permettant aussi l'exportation chiffrée et contextualisée des issues.

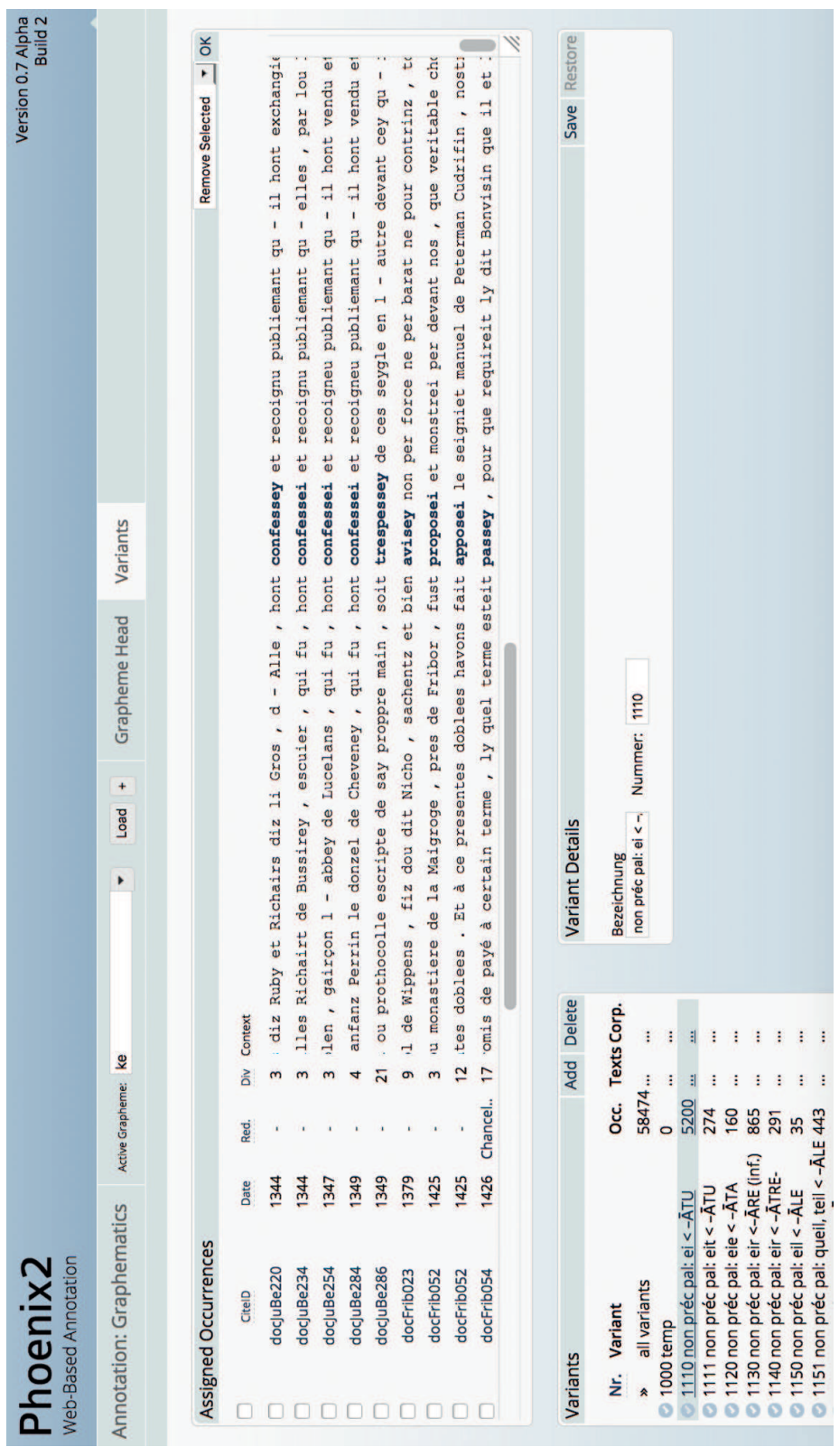

Ill. 3 : La catégorisation des formes grapho-phonétiques relevées dans phoenix. 
La reproduction de l'écran laisse entrevoir les dimensions quantitatives de nos relevés : pour 'A[ précédé ou non d'une consonne ou d'un groupe palatal, nous avons réuni plus de 58000 occurrences distribuées dans les différentes catégories retenues. ${ }^{51}$

Ces dernières prennent en considération (i) la base étymologique des formes et (ii) la physionomie des issues vernaculaires. D'un point de vue étymologique (=i), nous avons distingué selon les environnements pertinents, les formes provenant de -ATU, -ATA, -ARE, -ATRE et -ALE. ${ }^{52}$ Pour les noms, nous avons regroupé pour l'essentiel les pluriels avec les singuliers, sauf si le marquage sigmatique entraînait d'autres conséquences grapho-phonétiques.

Quant aux issues (= ii), nous avons distingué essentiellement quatre types principaux, chacun comportant un certain nombre de variantes :

$\langle\mathrm{a}\rangle=$ le type francoprovençal [a]

〈é〉 = le type oillique parisien 〈és [e] (qui a fini par s’imposer dans la norme du français)

$\langle e \mathrm{i}\rangle=$ le type oïlique régional ['e(i)]

〈ié〉 〈ia〉 = les types oïlique et francoprovençal après palatale ['je 'ja]

Nous avons finalement exploité 74 des 82 catégories retenues dans la base de données - huit se sont avérées trop peu nourries ou trop peu cohérentes -, en les regroupant en 38 ensembles qui nous ont semblé pertinents pour notre interrogation. Nous avons ensuite exporté les données chiffrées détaillées pour les 22 corpus individuels des DocLing et catégorisé les 836 interfaces obtenues dans une quinzaine de tableaux selon les bases étymologiques et la présence ou non d'un groupe ou d'un son palatal. Nous renonçons ici à la reproduction de cette matière épineuse $\mathrm{e}^{53}$ en présentant par la suite des tableaux simplifiés dont la lecture reste ardue.

Comme cela ressort déjà de cette brève description, la catégorisation résulte de nombreux sondages et tentatives de structuration préliminaires. Notre analyse des chartes de la Meurthe-et-Moselle distingue ainsi 31 catégories pour ce même paramètre (Glessgen 2008, 473-477). Les données actuellement à l'étude, plus nombreuses et plus diversifiées ont donné lieu à de nouvelles catégories, mais ont également conduit à une révision des conditions d'environnement. Nous avons

51 Les différents balisages effectués par nous dans les années 2006 à 2012 ont été réalisés avec la première version de phoenix (programmée avec tustep) et ont pu être exportés en 2016 dans la base my-sql du logiciel actuel phoenix-2 (v. n. 9).

52 Nous avons également retenu une catégorie pour des noms divers (CLAVE, MASU, MARE etc.), sans toutefois l'exploiter ici puisqu'ils ne permettaient pas une quantification à travers le territoire.

53 Les tableaux détaillés ont été déposés sur le site des DocLing. 
notamment dû revoir, pour les issues après consonne ou groupe palataux, les environnements de 'A [ qui provoquaient l'effet de Bartsch menant au dégagement d'une semi-consonne / $\mathrm{j} /$ et donc à l'apparition d'une diphtongue de coalescence (cf. Pierret 1994, § 330). Ce n'est qu'à travers la phénoménologie observable pour chacun des lexèmes individuels que nous avons pu établir une catégorisation qui nous semble désormais cohérente.

Voici les bases lexicales essentielles de notre corpus pour lesquelless nous avons pu constater l'effet de Bartsch, bases structurées d'après leur environnement dans l'ancienne langue :

[d3] adjugier, aubergier/hebergier, chalongier, changer, chargier (dechargier), corrigier, costengier, desdomagier, (e)changier, (en)gagier, jugier, ligiee, logier, losengier, obligier, ostagier, pargiee, vendangier

[n] signier (assignier, consignier, designier), acompagnier/compagnie(e), dignier ('dîner'), eloignier, enseignier, gagnier, seignier

[i/iz] aiguisier, aisié, amaisier, apaisier, baptisier, brisier, croisier, prisier

$[\mathrm{s}][<\mathrm{x} \sim \mathrm{pt} \sim \mathrm{sj}]$ baissier (<BASSIARE), chassier, engrossier, laissier (delaissier), texier, taxier

[ts] [ $<\mathrm{tj}$ etc.] adrecier/adressier, adoucier, annoncier (denoncier, noncier), chauciee ( $<\mathrm{CAL}$ CEARE), comencier, effacier, enforcier/efforcier, fiancier, justicier, obicier, percier ( $<{ }^{\star}$ PERTUSIARE), prononcier, renoncier, sentencier, trefoncier

[t] attachier, approchier, chevauchiee, depechier, duchiee, embauchier, empechier, eveschie, fauchier, marchié, pechier, touchier (atouchier)

$[\lambda]$ apareillier, baillier, conseillier, depouillier, detaillier, peilliee, perillier, taillier, travaillier

[i/ir] desclairier ( $<{ }^{\star}$ DISCLARIARE), dechirier ( $<{ }^{\star}$ skerjan), empirier ( $<$ PEIOR)

[i/it] amitié, esploitié, moitié, haitier (deshaitiez), rafaitier, retraitier, v(u)idier

[id] aidier, (em)plaidier

Or, ces issues se superposent largement dans leur physionomie avec celles qui résultent de la combinaison entre $/ \mathrm{i}$ j/ et les issues de ${ }^{\mathrm{A}}$ [ dans les lexèmes suivants de notre corpus :

aproprier, beneficier (latinisme), certifier, charroyer, copier, crier, deguier ('guider'), delaier, edifier, emploier, enlier, envoier (renvoyer), essayer, excommunier, guerroyer, injurier, justifier, lier (relier), maisniee, marier (remarier), multiplier, nettoyer, notifier, obvier, oublier, o(c)troyer, pacifier, paier, pledoyer, prier, publier, ratifier, specifier, supplier, verifier

Cet ensemble rejoint, toujours d'un point de vue physionomique, quelques lexèmes où 〈i $\sim$ y reflète la rupture phonique du hiatus : agreier, alloyer pour alloer (+ loyer pour loer) ainsi que le régionalisme avoyé (+ desavoyee). ${ }^{54}$

54 Par sécurité, nous avons également établi une liste de lexèmes qui ne montrent pas l'effet de Bartsch, mais qui pourraient induire en erreur lors du balisage: accenser, amasé (< ${ }^{\star} \mathrm{MASU}$ ), annexer, arraser, aviser, casser, cesser, coser (cf. coudre Codi), confesser, depenser, deviser/diviser, espouser, fossé, geter/jeter, passer, penser, poser (opposer, proposer), refuser. 
Ce n'est qu'après avoir clarifié la relation entre ces différents ensembles que nous avons achevé notre balisage en vue de son analyse scriptologique.

\section{5 Étude de cas : les issues de 'A[ non précédé de palatale entre scriptae francoprovençales et françaises}

Nous prendrons d'abord appui sur les bases étymologiques (-ATU, -ATA, -ARE, -ATRE, -ALE) pour présenter leurs issues dont la distribution peut varier considérablement dans un même corpus en fonction de l'environnement concret. Dans un deuxième temps, nous synthétiserons et quantifierons les résultats obtenus pour les différentes bases. Ces deux opérations sont de nature macroscopique : nous considérons les corpus comme des entités à part entière, même s'ils ont été établis pour la plupart d'après les lieux de conservation des documents. En cas d'incohérences, nous prendrons toutefois aussi en considération les lieux d'écriture du territoire francoprovençal.

Dans tous les tableaux, nous avons mis en relief la distribution des variantes de manière typographique :

- gras + rouge : $>60 \%$ des occurrences pour une région

- gras et italique + bleu : formes alternatives avec un pourcentage semblable (40-60\%)

- italique + bleu : $30 \%-39 \%$

- $\quad$ gris : formes secondaire avec une certaine présence (4-29\%)

\section{1 -ATU non précédé de palatale (20446 occ.)}

Les issues de -ATu non précédé de palatale représentent l'ensemble de loin le plus nombreux dans nos corpus avec plus d'un tiers des occurrences relevées. Ce relevé vaste et varié permet de cerner les grandes lignes interprétatives autant concernant la relation qui s'instaure entre les scriptae oïlique et francoprovençale que certaines particularités de chacune d'entre elles. Le tableau suivant fait ressortir notamment la distribution entre le type francoprovençal 〈a〉 et les deux types oïliques 〈é eì : 


\begin{tabular}{|c|c|c|c|c|c|}
\hline Corpus & 〈a〉 & 〈ei eit〉 [riei〉] & 〈é〉 & 〈ié» & total \\
\hline Codi & 371 & 4 & 0 & 0 & 375 \\
\hline Grenoble & 238 & 0 & 3 & 0 & 241 \\
\hline Lyonnais & 1397 & 7 & 152 [10\%] & 0 & 1556 \\
\hline Ain & 227 & 3 & 114 [33\%] & 0 & 344 \\
\hline Forez & 706 & 2 & 387 [35\%] & 0 & 1095 \\
\hline Fribourg & 165 [12\%] & 645 [46\%] & 583 [42\%] & 0 & 1393 \\
\hline Vaud/Genève & 73 [12\%] & 114 [18\%] & 432 & 15 & 634 \\
\hline $\begin{array}{l}\text { Neuchâtel } \\
\text { [NCh] }\end{array}$ & 102 [15\%] & 77 [11\%] & 489 & 10 & 678 \\
\hline $\begin{array}{l}\text { Bourgogne, } \\
\text { Jura [FR] }\end{array}$ & 8 & 122 [4,5\%] & 2562 & 66 & 2760 \\
\hline Jura $[\mathrm{CH}]$ & 1 & 1130 [40\%] & $1657[58 \%]$ & 57 & 2845 \\
\hline Doubs [NCh] & 1 & 308 [31\%] & 583 & 88 [9\%] & 980 \\
\hline HSaône & 2 & $161[17 \%]$ & 723 & $52[6 \%]$ & 938 \\
\hline $\begin{array}{l}\text { Vosges, } \\
\text { HMarne }\end{array}$ & 0 & 736 [40\%] & 1046 [58\%] & 71 & 1853 \\
\hline $\begin{array}{l}\text { Meuse, MMo- } \\
\text { selle }\end{array}$ & 0 & 1510 [72\%] & 583 & 0 & 2097 \\
\hline Douai, Marne & 0 & 651 [37\%] & 1119 & 5 & 1775 \\
\hline ChRoy & 0 & 9 & 874 & 1 & 884 \\
\hline total & 3293 & 5479 & 11307 & 368 & 20446 \\
\hline
\end{tabular}

Le tableau, à première vue difficile à comprendre, permet un nombre important d'observations. D'abord un constat concernant la scripta oïlique : celle-ci montre une alternance forte entre le type 〈é〉 et le digramme 〈ei〉 (voire 〈eit〉), alternance qui n'était pas prévisible dans ces dimensions sur la base es ouvrages de référence en phonétique historique (cf. infra 6.2). Plus en détail :

- Au sein de la chancellerie royale, 〈é〉 est exclusif. En effet, la variante 〈ei〉 n'apparaît que dans deux des huit actes picardisants, $1 \mathrm{x}$ dans un acte émis à Paris (RP 12970320 02), 8x dans un acte rédigé à Lille (RP 12970829 01). Les actes véritablement parisiens comportent donc le type 〈é`sans aucune va- 
riance. La Bourgogne, avec sa scripturalité vernaculaire peu développée et tardive s'inscrit essentiellement dans cette même logique.

- Dans tous les autres territoires d'oïl en revanche, le digramme uei est très présent, autant en Picardie qu'en Champagne, en Lorraine et en FrancheComté (avec Porrentruy [Jura/CH]). En dehors de la HSaône (17\%), elle représente partout entre 30 et 70\% ; elle est dominante en Meuse et MMoselle et se trouve en équilibre avec 〈é` en HMarne, dans les Vosges et à Porrentruy.

Nous reviendrons sur ce constat (cf. infra 6.2). Dans l'immédiat, nous pouvons retenir que les types oïliques 〈é ei〉 s'opposent clairement au type francoprovençal 〈a〉. Ce dernier, quant à lui, est exclusif dans le Dauphiné, presque général dans le Lyonnais et dominant dans le territoire francoprovençal à l'Ouest et au Nord de Lyon ; il est minoritaire, mais bien présent dans le territoire francoprovençal en Suisse (Neuchâtel, Vaud/Genève, Fribourg : 12-15\% des occurrences). Nous trouvons ici la bipartition de la scripta du territoire francoprovençal qui oppose les régions de France et de Suisse. Les premières connaissent une scripta fortement sinon pleinement francoprovençalisante, les secondes une scripta de type oïlique incluant des éléments francoprovençaux plus ou moins marqués. Retenons d'abord trois observations plus générales dans ce contexte :

(1) En France, les issues francoprovençales sont non seulement dominantes, comme ce premier tableau le laisse entrevoir, mais elles sont presque généralisées jusqu'aux alentours de 1360 : avant, à Lyon 95\% des occurrences correspondent à 〈a〉, dans le Forez 100\% et dans l'Ain encore 70\% (cf. infra 6.1) ; les deux ensembles dauphinois se plaçant avant ce moment charnière, la présence exclusive de «a〉 s’inscrit dans cette même logique chronologique.

(2) En Suisse, Fribourg représente une entité à part. Dans le cas présent, la scripta de cette ville se place à mi-chemin entre la scripta oïlique sudorientale (Vosges, HMarne, Porrentruy, Doubs) et la scripta francoprovençalisante des régions suisses voisines (Neuchâtel, Vaud, Genève). D’un point de vue physionomique, elle fait la transition entre ces dernières et les régions francoprovençales de France : le pourcentage de ‘é̀ est très semblable entre Fribourg (42\%), l'Ain (33\%) et le Forez (35\%), mais bien plus faible qu'à Neuchâtel (72\%) et Vaud/Genève (68\%) ; simplement, Fribourg retient dans une mesure importante le type régional oïlique «ei〉 à côté du type francoprovençal, dominant dans le groupe lyonnais. La position de Fribourg ressortira plus nettement encore des prochains paramètres.

(3) Enfin, la scripta francoprovençalisante de Suisse se place dans la continuité immédiate des régions oïliques voisines : Neuchâtel, le pays de Vaud et la région de Lausanne et Genève sont proches de la scripta franc-comtoise (et, 
comme nous le verrons plus loin, bourguignonne), ici par la présence significative de eì (11/18\%) et, dans une moindre mesure, par les apparitions ponctuelles du type 〈ié> (cf. infra). En revanche les éléments oïliques de la scripta francoprovençale autour de Lyon sont avant tout de type parisien et ne relèvent pas d'une scripta oïlique régionale. Lyon s'oriente donc vers Paris, et non vers Dijon ou Besançon.

Au-delà de ces constats «macroscopiques », le relevé des quelque 20000 occurrences appelle six remarques plus ponctuelles concernant les différentes issues concrètes :

(1) 〈a〉: le type francoprovençal fait apparition, de manière très ponctuelle, dans certains actes des régions oilliques sud-orientales :

- à Dijon (1 occ. dans chCOr nº 190 [Saint Bénigne de Dijon] et les copies 191, 191a)

- à Theuley-l'Abbaye (2 occ. dans chHS $n^{\circ} 77$, d'une forme linguistique particulière [elle comporte aussi la variante «-iet» après palatale, cf. infra 7.1])

- $\quad$ à Besançon (1 occ. dans chNCh no 142) et à Porrentruy (1 occ. dans docJurBern n $\left.{ }^{\circ} 41\right)$

- dans un acte légèrement francoprovençalisant impliquant un noble de Savigny ${ }^{55}$ (1 occ. de pra $<-$ ATU $^{56}$ dans chSL n ${ }^{\circ} 33$ )

- $\quad$ enfin dans deux actes du Jura dans une scripta plus ou moins francoprovençalisante (3 occ. dans chJu n ${ }^{\circ} 13$ [abbaye de Saint-Claude dans le massif du Jura] et 1 occ. dans chJu $\mathrm{n}^{\circ}$ 48)

Si le choix de cette forme n'est pas pleinement explicable dans tous les actes, ${ }^{57}$ elle se rattache toutefois pour l'essentiel à l'oral francoprovençal; il s'agit de toute évidence d'une marque très nette et perçue comme telle.

(2) 〈az〉 $v s\langle a s\rangle$ : le marquage sigmatique du type francoprovençal génère une différence légère, mais nette entre les régions de Suisse et de France. Dans tous les corpus de France, le type 〈-as〉 est pratiquement exclusif (l'on ne relève pour l'intégralité des actes qu'une seule occ. de «-az〉 à Lyon), alors que dans les régions de Suisse, 〈-az〉 domine nettement (7x NCh vs 0 occ. «-as〉, 8x Vaud/Genève vs 1x «-as〉, $87 \mathrm{x}$ Frib vs $15 \mathrm{x}$ «-as〉 dans 2 doc.).

Cela vaut également pour les quelques formes citées auparavant ( $«$-az〉 apparaît 3x dans chCOr n 190-191a, 1x dans chJu n 48 et $1 \mathrm{x}$ dans docJuBe 41, jamais 〈-as〉). Les rares marques francoprovençalisantes dans les actes franc-comtois (et

55 La rédaction de lacte est éventuellement due à l'abbaye de Savigny près de Lyon.

56 Cf. aussi mua <-'ATA [cf. infra] ; enquesta [bis] <-'ARE [cf. infra] ; ordra 'ordre', quarta <'-A. Cf. infra 5.3.(3).

57 Nous avons supprimé l'occurrence «mandaz et commandanz [...] » (ChN 21, 43) : le manuscrit est univoque, mais la syntaxe l'est également et il faut supposer un trait de nasale oublié audessus de mandaz. 
bourguignons) témoignent donc d'une interaction plus forte de leur scripta avec les régions de Suisse qu'avec celle en usage autour de Lyon.

(3) $\langle e y(s)\rangle v s\langle e i(s)\rangle^{58}$ : la variance graphique entre $\langle y\rangle$ et $\langle\mathrm{i}\rangle$ est rarement significative d'un point de vue scriptologique; ici toutefois, l'on distingue très nettement quatre espaces :

- $\quad$ en Picardie, en Champagne et en Lorraine «-ei(s)〉 est généralisé (aucune occ. de 〈-ey(s)〉 à Douai, 84 occ. dans les cinq départements champenois et lorrains vs 2555 occ. de 〈-ei(s) > [= 3\%]);

- $\quad$ en Bourgogne, dans la Haute-Saône et dans le Jura français, «-ey(s)〉 représente 32\% des occurrences (122 vs 444 <-ei(s)〉) ;

- le pourcentage augmente encore dans les territoires oïliques adjacents au domaine francoprovençal de Suisse qui se détachent ainsi du reste de la Franche-Comté : 51\% à Porrentruy ( 578 vs 552 occ.) et $73 \%$ à Besançon (226 vs 82 occ.) ;

- $\quad$ cette logique se poursuit en territoire francoprovençal : 44\% à Fribourg (288 vs 357 occ.), 64\% à Neuchâtel (49 vs 28 occ.) et 90\% dans le pays de Vaud, à Lausanne et Genève (103 vs 11 occ.).

Ce constat s'explique probablement par le rejet du graphème 〈i en fin de mot, phénomène déjà observé pour les textes de la Bourgogne par Philipon (1910, 506s.). Ce rejet peut être motivé par la fonction morphologique du graphème dans les textes latins ${ }^{59}$ ou encore, en territoire francoprovençal, par le fait que 〈i final peut avoir une valeur phonétique (cf. supra ivoiglli, pecheri, riveri). Voici un relevé des occurrences concernées dans notre corpus :

\begin{tabular}{lccr}
\hline & $\langle$ ei & $\begin{array}{c}\text { 〈ey eis eiz [eit] }\rangle^{60} \\
\text { ‘eyz/eys }\end{array}$ & total \\
\hline Territoire d'oïl hors sud-est & 2798 & $1191[=30 \%]$ & 3989 \\
\hline Bourgogne, Franche-Comté & 55 & $66[=54,5 \%]$ & 121 \\
\hline $\begin{array}{l}\text { Territoire francoprovençal en } \\
\text { Suisse }\end{array}$ & 122 & $949[=88,5 \%]$ & 1071 \\
\hline $\begin{array}{l}\text { Territoire francoprovençal en } \\
\text { France }\end{array}$ & $\varnothing$ & $16[=100 \%]$ & 16 \\
\hline
\end{tabular}

Si dans le nord et l'est du territoire d'oïl, la réticence à l'égard de 〈i en fin de mot est faible, elle augmente nettement dans le sud-est et encore plus en territoire

58 Le type «-eit» ne comporte jamais le graphème 〈y〉.

59 Cf. Carles (2011, 514-516).

60 Le type «ei dans la Somme du Code correspond concrètement à 4 occ. de «-eit». 
francoprovençal. En France, le type 〈ei〉 est certes inhabituel, mais les seize occurrences sporadiques évitent toutes la position finale de 〈-i〉. Ce phénomène est d'autant plus notable en domaine francoprovençal qu'il s'agit ici de la gestion particulière d'une variante grapho-phonétique typique de la scripta oïlique.

(4) 〈eit〉 : cette variante graphique dans le type diphtongué caractérise surtout la scripta picarde (Douai), dans une moindre mesure la Champagne et la Lorraine septentrionales : sur les 274 occurrences de «-eit 176 sont de Douai et 83 de la Marne, de la Meuse et de la MMoselle ; à Douai cela correspond à un quart des occurrences pour ce paramètre ( $27 \%$ de 660 occ.) et à $71 \%$ des formes comportant la diphtongue (176x «eit〉 vs $73 \mathrm{x}$ 〈ei $)$. Dans les autres régions oïliques et francoprovençales, le phénomène est pratiquement absent; il s'agit donc bien d'un régionalisme graphématique à l'intérieur du domaine d'oïl. ${ }^{61}$

(5) 〈iei〉: le trigramme est une variante très rare de 〈ei〉 avec 2 occ. dans le corpus de la MMoselle (dans des documents dont les rédacteurs sont le comte du Luxembourg et l'évêque de Verdun) et 3 occ. en HMarne (l'abbaye de Septfontaines près de Chaumont et l'église de Chateauvillain au sud-ouest du département). Les quatre actes concernés se placent sur un axe Nord-Sud entre le Luxembourg, le nord de la Meuse et le sud de la HMarne, mais il s'agit néanmoins d'un épiphénomène qui illustre le rejet des trigrammes par les scribes, voire des triphtongues par les locuteurs.

(6) 〈ié〉 : cette variante, à première vue surprenante, se concentre en FrancheComté et est faiblement présente dans les régions adjacentes. Elle reste circonscrite pour l'essentiel à un nombre restreint de lexèmes : les trois mots curié (et var., 187 occ.), jurié (et var., 111 occ.) et quittié (et var., 36 occ.) se font fort de 90\% des occurrences du phénomène (334 sur 368 occ.). Il faut sans doute supposer un effet d'assimilation à partir de [y i] prétonique, évolution phonétique bien attestée pour ces trois lexèmes en Franche-Comté et en Lorraine dans une frange Nord-Sud allant de la région de Metz jusqu'à celle Montbéliard, puis en Jura bernois. $^{62}$

22 des autres 34 occurrences concernent acensié (et var.) et divisié, les 12 dernières se distribuent parmi les huit lexèmes suivants : aimiez, annexié, apeliez, aterié, descomblié, exceptié, meusuriez. À côté des quelque 11000 occurrences pour 〈é`, ces variantes appellent des explications particulières ; il peut s'agir tantôt d'un phénomène phonique (probable dans divisié et meusuriez), d'un lapsus calami (par ajout d'un jambage) ou d'une interférence quelconque (cf. les

61 Il faut distinguer de ce trait la variante rarissime «-iet` qui apparaît de manière sporadique (3 occ. dans 2 actes) dans les chartes de la HSaône (à côté de 49 occ. de (iés).

62 Cf. FEW 5, 80a; 81a s.v. JURARE : [3y'ri(ə)] 'jurer’; ib. 2/2, 1558a s.v. cuRA : [ky'ri] ; ib., 1559a s.v. CURARE : [kœ'rjœ] 'curer'; ib., 1474a; 1475 a s.v. QUIETUS : [ki'tje ki'ti]. 
seules occurrences dans les actes de la chancellerie royale: outroié, quittié et deleissié [ChRoy 1298/10/32] où l'interférence pourrait être motivée par le contexte des lexèmes environnants).

Sur la base de notre analyse il devient non seulement possible de reconnaître les grandes lignes de diffusion et de distribution des diverses issues et variantes grapho-phonétiques en question, mais aussi de cerner avec précision les différentes strates linguistiques coprésentes dans les documents du territoire francoprovençal. Si les différents sous-ensembles montrent des attitudes bien distinctes, nous sommes très souvent en face d'une situation de «mixité » entre variables de type francoprovençal et oïlique.

Grâce à l'analyse structurelle, les textes concrets pourront désormais être mieux interprétés dans leur compositionnalité. L'approche quantificatrice fait ressortir que les éléments reconnaissables «à l'œil nu » dans les textes individuels s'inscrivent dans des logiques sous-jacentes et ne sont pas le fait du hasard ou de choix idiosyncrasiques des scribes.

Nous présenterons par la suite de manière plus succincte les résultats de ${ }^{A} \mathrm{~A}$ dans -ATA, -ARE, -ATRE et -ALE en relevant surtout les différences par rapport aux résultats de -ATU.

\section{2 -ATA non précédé de palatale (8475 occ.)}

Le sous-ensemble de -ATA(s) est moins fréquent que celui de -ATu réunissant toutefois encore près de 8500 occurrences. Le relevé synthétique montre certaines différences - notamment une plus faible diversité des issues - mais il concorde avec -ATU sur les trois points principaux :

- la prédominance de 〈a〉 dans la scripta francoprovençale et de «ee〉 dans la scripta oïlique ;

- la différence considérable entre le territoire francoprovençal de France (où le type 〈a〉 est encore plus présent sinon exclusif) et de Suisse (où le type «a est moins marqué [avec $2 \%, 7,5 \%$ et $11,5 \%$ ] que pour -ATU [12-15\%]), au moins jusqu'à la deuxième moitié du $14^{\mathrm{e}}$ siècle (les formes en «ee〉 du Forez ne sont pas contemporaines des formes en «a`, mais postérieures, cf. infra 6.1);

- la présence du type diphtongué représenté par «eie» qui est toutefois géographiquement plus circonscrit que pour -ATU (seulement en Lorraine et en Champagne méridionale et presque pas en Picardie et dans le Sud-Est oïlique). Sa présence est également beaucoup plus réduite en nombre ( $11 \%$ en Lorraine et en Champagne vs 40-70\% dans la même région pour -ATU). 
Avant d'en venir aux observations de détail, voici le tableau de synthèse qui comporte des regroupements plus forts que celui de -ATu à cause de sa moindre variance :

\begin{tabular}{|c|c|c|c|c|}
\hline Corpus & $\begin{array}{c}\text { «a aa ae〉 } \\
\text { «as az ais ays } \\
\text { aes ayes〉 }\end{array}$ & 〈eie〉 & $\begin{array}{c}\langle e e\rangle \\
{\left[\langle i e e\rangle^{63}\right]}\end{array}$ & total \\
\hline Dauphiné & 586 & 0 & 0 & 586 \\
\hline Lyonnais & 278 [89\%] & 0 & 36 [11\%] & 314 \\
\hline Ain & 96 & 0 & 0 & 96 \\
\hline Forez & $361[62,5 \%]$ & 0 & $216[37,5 \%]$ & 577 \\
\hline Fribourg & 206 [11,5\%] & 0 & 1596 & 1802 \\
\hline Vaud/Genève & $15[7,5 \%]$ & 1 & 185 & 201 \\
\hline Neuchâtel [NCh] & $4[2 \%]$ & 0 & 207 & 211 \\
\hline Franche-Comté, Bourgogne & 3 & 18 & 2526 & 2547 \\
\hline Champagne, Lorraine & 0 & 127 [11\%] & 1018 & 1145 \\
\hline Douai, Marne & 0 & 14 & 629 & 643 \\
\hline ChRoy & 0 & 0 & 353 & 353 \\
\hline total & 1549 & 160 & 6766 & 8475 \\
\hline
\end{tabular}

Dans la comparaison avec les issues de -ATu l'on constate d'abord la faiblesse du type «eiè en domaine d'oïl, puis une variance particulière à l'intérieur du type francoprovençal.

〈eie〉: la présence fortement réduite de ce type souligne peut refléter autant un rejet des scribes à l'égard des trigraphes, qu'un rejet des triphtongues de la

63 〈iee〉 : le type est pratiquement absent du tableau, contrairement à 〈ié〉 auparavant. Nous n'avons relevé que 4 occurrences sur les 6762 de 〈ees: la forme getiee à deux reprises dans une charte des ducs de Lorraine ( $\left.\mathrm{chV} \mathrm{n}^{\circ} 114\right)$ et la forme devisiee(s) à la fois dans un acte de l'abbaye de Saint-Martin d'Autun (chSL $\mathrm{n}^{\circ}$ 17) et dans un vidimus royal (RV 1287/08/32_01c). Le premier reflète de nouveau une forme phonétique régionale (le type ['zti dze'ti] est bien attesté en Lorraine méridionale, cf. FEW 5, 12b s.v. JACTARE). Le deuxième rejoint le groupe divisié relevé plus haut. La grande rareté de ces formes par rapport à 〈ié〉 peut partiellement être due aux aléas de la distribution lexicale puisqu'il s'agit d'un phénomène phonétique plus lié à des lexèmes individuels que reflétant une tendance macroscopique généralisée. 
part des locuteurs. Le phénomène phonétique sous-jacent nous semble toutefois primordial, et ses effets peuvent être renforcés par l'attitude des scribes. Il est vraisemblable que la triphtongue pour -ATA a pu se former dans les mêmes régions que la diphtongue pour -ATU, mais elle s'est réduite beaucoup plus tôt que cette dernière.

〈a〉: l'analyse du type francoprovençal nécessite un relevé détaillé des différentes variantes, 〈a aa ae aye ainsi que les formes avec une finale sigmatique flexionnelle. En voici le tableau qui précise ces issues réunies dans le tableau synthétique :

\begin{tabular}{|c|c|c|c|c|c|c|c|c|c|c|}
\hline & 〈a〉 & $\langle a \mid\rangle$ & $\langle a e\rangle$ & «aye & $\langle a s\rangle$ & $\langle a z\rangle$ & $\begin{array}{r}\text { ‘ais } ~ \\
\text { ays〉 }\end{array}$ & 〈aes〉 & 〈ayes〉 & total \\
\hline Codi & 128 & 0 & 2 & & 2 & & 33 & & & 165 \\
\hline Grenoble & 286 & 0 & 0 & & 2 & & 133 & & & 421 \\
\hline Lyonnais & 260 & 9 & 0 & & 2 & 1 & 5 & & 1 & 278 \\
\hline Ain & 81 & 0 & 0 & & 2 & & 11 & 2 & & 96 \\
\hline Forez & 253 & 28 & 0 & & & & 80 & & & 361 \\
\hline Fribourg & 45 & 0 & 26 & 9 & & 2 & & 120 & 4 & 206 \\
\hline Vaud/Genève & 0 & 0 & 0 & & 1 & & & 14 & & 15 \\
\hline Neuchâtel [NCh] & 0 & 0 & 2 & & & & & 2 & & 4 \\
\hline total & 1053 & 37 & 30 & 9 & 9 & 3 & 262 & 138 & 5 & 1546 \\
\hline
\end{tabular}

Le premier constat reste que le type 〈a〉 est très fortement présent en domaine francoprovençal de France ; nous verrons en effet qu'il se maintient à Lyon une décennie plus longtemps que dans le cas de -ATu (cf. infra 6.1). A priori, ce constat s'explique facilement puisque dans -Ata l'issue de 'a[ se superpose et fusionne avec celle de -A final inaccentué (conservé en francoprovençal, cf. supra 3.1) ; la succession des deux éléments est même rendue graphiquement par ‘aa` en Forez et, ponctuellement, à Lyon.

Il est plus surprenant d'observer qu'en domaine francoprovençal de Suisse, le type francoprovençal est notablement moins fréquent que pour les issues -ATU ; c'est donc une tendance opposée qui se manifeste ici et dont l'explication est plus difficile. Le plus probable nous semble que le phénomène prend appui sur la notation «-aes〉 du féminin pluriel qui est, quant à lui, parfaitement en cohérence avec l'évolution phonétique. En effet, en francoprovençal, -A final inaccentué 
devant [-s] s'affaiblit en [-ə], comme en français (cf. supra 3.1). Or, dans la scripta fribourgeoise et même neuchâteloise le pluriel en 〈aes` a donné lieu par analogie à une forme de singulier non sigmatique, 〈ae aye〉, suite à l'amuïssement des consonnes finales (cf. supra 3.2.1). Étant donné que ce digramme (voire trigramme) est fréquent, il est possible qu'il ait pu être rapproché de la forme oïlique 〈ee〉 qui s'est alors imposée. Notre argumentation est renforcée par le fait que la forme 〈aes〉 issue de -ATA n'est pas moins fréquente que les formes en 〈az as〉 provenant de -ATU (cf. supra : 7 formes sigmatiques à Neuchâtel, 9 dans le pays de Vaud, Lausanne et Genève, 102 à Fribourg, sachant qu'il y a plus de lexèmes qui sont concernés par -ATU que par-ATA). Le pluriel reste donc intact et c'est le type singulier qui est sujet à la fusion avec le type oïlique. ${ }^{64}$

Indépendamment de cette tentative d'explication, la divergence entre la France et la Suisse est patente et montre bien deux traditions scripturales distinctes. La différence porte également sur les formes du pluriel, où le type suisse 〈aes〉 s'oppose au type de France 〈ais ays〉. Seul Fribourg représente une transition entre les deux territoires, au moins pour les formes non sigmatiques.

\section{3 -ARE non précédé de palatale (6097 occ.)}

Les notations des issues de -ARE sont toujours relativement nombreuses et rejoignent pour l'essentiel celles de -ATA :

- 〈ar〉 domine en territoire francoprovençal de France (le cas du Forez s'explique de nouveau par des raisons chronologiques, cf. 6.1) ;65

- «er» domine en territoire d'oïl et en Suisse, sauf à Fribourg qui représente de nouveau une situation de transition entre la zone francoprovençale de Suisse et de France ;

64 Notons que le type 〈a〉 ne fait pratiquement pas apparition dans les actes du Sud-Est oïlique, contrairement à ce qu'on a pu observer pour -ATU. La très faible présence de «a non sigmatique en Suisse peut s'expliquer par le fait que ce type n'a pas connu, ci et là, des retombées dans des actes en territoire d'oïl, impliquant d'une manière ou d'une autre des personnes ou des biens du domaine francoprovençal. Les deux seules occurrences relevées appartiennent aux deux docu-

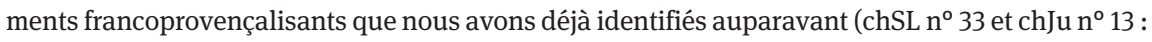
discorde fust mua).

65 Pour l'Ain, 9 des 13 occ. de ser appartiennent à un seul document, ce qui réduit à $9 \%$ la présence du type dans le reste du corpus. 
- le type oïlique régional «eir eyr〉 est moins présent que pour -ATU, mais plus que pour -ATA, toujours en Champagne et en Lorraine ${ }^{66}$ et également en Suisse ;

- $\quad$ ier est pratiquement absent. ${ }^{67}$

Voici le tableau synthétique :

\begin{tabular}{|c|c|c|c|c|}
\hline Corpus & 〈ar & 〈eir eyr〉 & 〈er〉 [〈ier〉] & total \\
\hline Lyonnais, Dauphiné & 1245 & 0 & $51[4 \%]$ & 1296 \\
\hline Ain & 32 & 0 & 13 [29\%] & 45 \\
\hline Forez & $95[41,5 \%]$ & 7 & $127[55,5 \%]$ & 229 \\
\hline Fribourg & 249 [23\%] & $451[41,5 \%]$ & $385[35,5 \%]$ & 1085 \\
\hline Vaud/Genève & $3[2 \%]$ & 23 [16\%] & 115 & 141 \\
\hline Neuchâtel [NCh] & 0 & 1 & 89 & 90 \\
\hline Fr-Comté, Bourgogne & 1 & 10 & 1222 & 1233 \\
\hline Vosges, HMarne & 0 & 75 [16\%] & 387 & 462 \\
\hline Meuse, MMoselle & 0 & $254[39,5 \%]$ & $388[60,5 \%]$ & 642 \\
\hline Douai, Marne & 0 & $44[7 \%]$ & 581 & 625 \\
\hline ChRoy & 0 & 0 & 248 & 248 \\
\hline total tout & 1626 & 865 & 3606 & 6097 \\
\hline
\end{tabular}

Sur la base de ces chiffres, on peut retenir trois observations, une graphématique, une grapho-phonétique et une de nature scriptologique :

(1) 〈eir〉: la graphie 〈eyr est relativement rare et n'apparaît qu'en territoire francoprovençal, autant de Suisse que de France. Elle est présente dans le pays de Vaud, Lausanne et Genève $(9 \mathrm{x}$ «ey $(\mathrm{r})>$ vs $16 \mathrm{x}$ «eir $)$ et dans le Forez (6x

66 Le corpus de Douai ne comporte que 4 occ. de «eir»; par ailleurs, 6 des 7 occ. du Forez proviennent d'un seul acte.

67 Les lexèmes en question sont très variés : en Marne, ils appartiennent à trois documents rédigés par la même abbaye (Igny, près de Reims : demandier, essartier, rapelier, chMa n ${ }^{\circ} 121,149$, 117 ) ; s'ajoutent amformier (chSL n ${ }^{\circ}$ 52) et pesturier (docJuBe 1). Les 5 occurrences ont été intégrées dans la colonne «er. 
〈eyr vs $1 \mathrm{x}$ 〈eir») ; s'ajoute une seule occ. de «ey〉 à Fribourg contre 455 occ. 〈eir〉). Le phénomène rejoint la graphie 〈ey〉 (<-ATU) dont la diffusion était toutefois plus large.

(2) absence de «r $r$ final : l'on constate un certain nombre de cas d'amuïssement de $[r]$ final après 〈é〉, 〈ey〉 et même 〈a〉: autant dans des actes oïliques du Sud et du Sud-Est oïliques (Meuse 1x, MMoselle 1x, V 1x, Ju 1x) que dans des actes francoprovençalisants de Suisse (Vaud/Genève 5x, Fribourg 3x, la charte $\mathrm{n}^{\circ} 33$ de la Saône-et-Loire 1x [cf. infra (3)]).

(3) 〈ar〉: les deux occurrences de $\langle\mathrm{a}(\mathrm{r})\rangle$ dans les corpus du territoire d'oïl proviennent de la charte SL n ${ }^{\circ} 33$ (enquesta) que nous avons déjà identifiée comme francoprovençalisante (cf. supra n. 52) ainsi que de la charte Ju n 3 qui l'est également (meisonar; cf. chartra [ter], Valclusa [bis]) et qui a aussi pu être rédigée par l'abbaye de Savigny de laquelle dépendait la chartreuse de Vaucluse (comm. de Cuiseaux), bénéficiaire de l'acte.

D'un point de vue interprétatif, il reste à expliquer la très faible présence de formes en «arı à Neuchâtel, en pays de Vaud ainsi qu'à Lausanne et à Genève. Elle est encore inférieure à celle des issues en 〈a〉 de -ATA ( 0 et 2\% contre 2 et 7,5\%) sans parler de celles pour -ATU (15 et 12\%). Cela contraste nettement avec les choix de Fribourg où la présence de «ar〉 atteint le double de «a issu de -ATU/-ATA (23\% contre 11,5 et $12 \%$ ).

On pourrait imaginer que les scribes de ces corpus aient agi dans un souci de cohésion des paradigmes verbaux, afin d'éviter des malentendus et garantir une certaine homogénéité des textes. Cet argument est néanmoins très fragile si l'on considère le choix opposé des scribes de Fribourg et, surtout, la mixité générale indéniable des scriptae en Suisse romande. Nous savons toutefois qu'une fine conscience linguistique a pu conduire les scribes d'autres régions galloromanes à faire des choix visant à respecter une certaine harmonie textuelle (cf. Carles 2011 pour l'Auvergne entre le $9^{\mathrm{e}}$ et le $11^{\mathrm{e}}$ siècle), sans pour autant que ces choix soient systématiques. Nous avons la conviction, à l'état actuel de nos analyses, que les marques francoprovençales suivent une logique interne, difficile à cerner et non exempte d'incohérences. Il est patent que certains traits de l'oral francoprovençal font surface à l'écrit avec une certaine régularité tandis que d'autres non. Le cas de 〈a〉 fournirait alors un argument pour supposer que le choix de ces traits est tributaire des traditions d'un lieu d'écriture donné - exactement comme on peut l'observer pour les marquages intra-oïliques en Lorraine au $13^{\mathrm{e}}$ siècle (Glessgen 2008) - et qu'il peut donc varier d'un endroit à un autre. 


\section{4 -ATRE non précédé de palatale (3224 occ.)}

Les substantifs en -ATRE ne concernent que trois lexèmes de notre corpus : mere, pere et frere 'frère ; moine'. Ceux-ci sont toutefois assez nombreux, étant donné l'importance des noms de parenté et des ecclésiastiques dans les actes. La distribution géolinguistique des types principaux s’inscrit dans les lignes identifiées auparavant :

- le type en 〈ar〉 domine de manière homogène dans tout le territoire francoprovençal de France (les formes en «er〉 sont de nouveau tardives, cf. 6.1) ;8 à Fribourg, il est aussi présent que les issues de -ARE (24 vs 23\%);

- $\quad$ ser montre une prédominance forte en territoire d'oïl et en Suisse romande ;

- la fréquence de la variante «eir est presque identique à celle des issues de ARE en domaine d'oïl (5/44/11\% vs 7/39.5/16\%) et à Vaud/Genève (11 vs 16\%), mais beaucoup plus réduite à Fribourg (6 vs 41,5\%), ce qui confirme notre hypothèse sur les logiques de choix dans un lieu d'écriture donné.

\begin{tabular}{|c|c|c|c|c|}
\hline Corpus & $\langle a r\rangle$ & 〈eir air & $\langle e r\rangle$ & total \\
\hline frpr. en France & 205 & 0 & $31[13 \%]$ & 236 \\
\hline Fribourg & 16 [24\%] & $4[6 \%]$ & 47 & 67 \\
\hline Vaud/Genève & $4[7,5 \%]$ & 6 [11\%] & 44 & 54 \\
\hline Neuchâtel [NCh] & 0 & 3 & 75 & 78 \\
\hline Fr-Comté, Bourgogne & 2 & 20 & 1173 & 1195 \\
\hline Vosges, HMarne & 0 & 71 [11\%] & 558 & 629 \\
\hline Meuse, MMoselle & 0 & $176[44 \%]$ & 227 & 403 \\
\hline Douai, Marne & 0 & $24[5 \%]$ & 457 & 481 \\
\hline ChRoy & 0 & 0 & 81 & 81 \\
\hline total & 227 & 304 & 2693 & 3224 \\
\hline
\end{tabular}

68 Elisabeth Berchthold a aimablement attiré notre attention sur le type (frauro» qui apparaît à une reprise dans les documents de Forez (1288). Il s'explique sans doute par analogie avec «fauros $<$ FABRU, fréquent à cette même époque et dans le même corpus (17 occ. dans deux documents de 1290). 
Une seule observation de détail : nous avons regroupé le type 〈air〉 avec 〈eir〉 dont il représente une variante, au moins en domaine d'oïl (MMoselle 2 occ. vs 80 occ. 〈eir», HMarne 2 occ. vs 62 occ. «eir», COr 1 occ. vs 8 occ. «eir», HSaône 2 occ. vs 6 occ. «eir», Jura 1 occ. [frayre]). Le cas est plus délicat pour Neuchâtel et Besançon, où les 4 seules occurrences de sair pourraient être interprétées comme des formes en interférence avec le type francoprovençal «ar».

\section{5 -ALe non précédé de palatale : QUALE / TALE (3734 Occ.)}

Les issues de QUALE et TALE avec leur différents composés et dérivés (lequel, itel etc.) sont bien présentes dans les actes, riches en phrases relatives et en éléments déictiques. Dans la distribution des différents types il faut prendre en considération la variante 〈quaul〉 à côté de «qual〉 où le maintien de 'A[ se superpose avec les effets de [1] implosif sur la voyelle précédente. Nous avons décompté les deux variantes séparément (deux colonnes dans le tableau), même si elles sont toutes les deux caractéristiques de la scripta francoprovençale :

- les types 〈al aul〉 ont une présence certaine à Neuchâtel et dans les pays de Vaud, Lausanne et Genève (7,5 et 9,5\%), alors qu'ils sont presque absents de Fribourg, ce qui est très surprenant. Dans les autres régions francoprovençales de France, 〈al aul〉 sont largement dominants, les formes en «el〉 du Forez étant toujours tardives (cf. 6.1) ;

- $\quad$ 〈el〉 domine partout en domaine d'oïl et en Suisse ;69

- la variante «eil s est présente en Champagne et Lorraine ainsi que, dans une faible mesure, en Suisse romande.

\begin{tabular}{|c|c|c|c|c|c|}
\hline Corpus & «quals & «quaul» & «queil teil» & 〈quel tel〉 & total \\
\hline Dauphiné & 121 & $80[40 \%]$ & 0 & 0 & 201 \\
\hline Ain, Lyonnais & 321 & $16[28 \%]$ & $21[3,5 \%]$ & $78[13,5 \%]$ & 582 \\
\hline Forez & $11[23 \%]$ & 3 & 0 & 34 & 48 \\
\hline
\end{tabular}

69 Nous avons placé ici les quelques formes de «queus teus` au pluriel (44 occ.) dont la distribution s'inscrit dans celle de «quel tel», avec seulement une concentration certaine à Douai (14x «-eus〉 vs $165 \mathrm{x}$ 〈-el)), dans la Marne (9x vs 176x «-el») et dans la chancellerie royale (7x «quieus〉 vs $139 \mathrm{x}$ «-el`); les autres 14 occurrences font surface de manière ponctuelle sans distinction géolinguistique par rapport à «-el`. 


\begin{tabular}{|c|c|c|c|c|c|}
\hline Corpus & squals & ‘quaul» & 〈queil teil〉 & 〈quel tels & total \\
\hline Fribourg & 0 & $10[2 \%]$ & $23[4 \%]$ & 496 & 529 \\
\hline Vaud/Genève & $17[7,5 \%]$ & $5[2 \%]$ & $24[11 \%]$ & 177 & 223 \\
\hline $\begin{array}{l}\text { Neuchâtel } \\
\text { [NCh] }\end{array}$ & $5[2,5 \%]$ & $10[5 \%]$ & 8 [4\%] & 173 & 196 \\
\hline
\end{tabular}

\begin{tabular}{lccccc}
\hline $\begin{array}{l}\text { Fr-Comté, } \\
\text { Bourgogne }\end{array}$ & 7 & 1 & 26 & 1792 & 1826 \\
\hline $\begin{array}{l}\text { Champagne, } \\
\text { Lorraine }\end{array}$ & 0 & 0 & $257[29,5 \%]$ & 617 & 874 \\
\hline Douai, Marne & 0 & 0 & $82[18 \%]$ & 364 & 446 \\
\hline ChRoy & 0 & 0 & 0 & 146 & 146 \\
\hline & & & & & \\
\hline total & 482 & 271 & 349 & 2632 & 3734 \\
\hline
\end{tabular}

\section{6 -ALE non précédé de palatale (3524 occ.)}

Les différentes bases nominales et adjectivales en -ALE sont plus difficiles à appréhender que QUALE/TALE ou encore que les affixes étudiés jusqu'ici. Dans ce groupe, les évolutions héréditaires à partir de bases latines, les formes savantes ainsi que les formations romanes se juxtaposent et sont difficiles à démêler. Après un premier relevé de formes en 〈al $\sim$ aul eil $\sim$ el〉, nous avons exclu les formations romanes évidentes (bannal, feal/foial, resal), de même que certaines formes savantes ou semi-savantes (annual, communal, corporal, hospital). Nous avons également exclu jornal dont les issues sont très complexes.

En résultat, nous avons gardé un ensemble non négligeable de 3500 occurrences où, malgré toutes les interférences et superpositions, le type phonétiquement attendu 〈el〉 domine très nettement en domaine d'oïl accompagné d'une présence régionale assez faible de 〈eil». Dans le territoire francoprovençal de France en revanche, le type 〈al aul〉 est pratiquement généralisé, à la seule exception du Forez. Ici, le type 〈al〉 est exclusif au $13^{\mathrm{e}}$ siècle, alors que 〈el〉 est généralisé dans les documents du $14^{\mathrm{e}}$ siècle (qui débutent toutefois tardivement, en 1358). 
Voici le tableau synthétique :

\begin{tabular}{|c|c|c|c|c|c|}
\hline Corpus & 〈alı & $\langle a u(l)\rangle$ & 〈eil〉 & 〈el〉 & total \\
\hline Lyonnais, Codi & 58 [42\%] & 72 [52\%] & 0 & 8 & 138 \\
\hline Ain, Grenoble & 22 & 1 & 0 & 0 & 23 \\
\hline Forez & 34 [47\%] & 0 & 0 & 38 [53\%] & 72 \\
\hline Fribourg & $16[7 \%]$ & 21 [9\%] & 0 & 190 & 227 \\
\hline Vaud/Genève & 31 [26\%] & $38[32 \%]$ & 1 & $50[42 \%]$ & 120 \\
\hline $\begin{array}{l}\text { Neuchâtel } \\
\text { [NCh] }\end{array}$ & 16 & 50 [52\%] & 0 & 30 [31\%] & 96 \\
\hline Jura $[\mathrm{CH}]$ & 917 & $72[6 \%]$ & 0 & $261[21 \%]$ & 1250 \\
\hline Doubs [NCh] & 67 [23\%] & $13[4 \%]$ & 0 & 210 & 290 \\
\hline $\begin{array}{l}\text { Bourg., Fr- } \\
\text { Comté, Vosges }\end{array}$ & 137 [16\%] & 113 [13\%] & 52 [6\%] & 572 & 874 \\
\hline $\begin{array}{l}\text { Champagne, } \\
\text { Lorraine }\end{array}$ & 36 [12\%] & 15 & 33 [11\%] & 208 & 292 \\
\hline ChRoy, Douai & 0 & 2 & 7 & 133 & 142 \\
\hline total & 1334 & 397 & 93 & 1700 & 3524 \\
\hline
\end{tabular}

La grande surprise concerne le territoire suisse, y inclus très exceptionnellement le Jura bernois avec Porrentruy: les formes francoprovençales concernent ici entre 60 et $80 \%$ des occurrences. Cette dimension dépasse tout ce que nous avons pu observer jusqu'ici. Il est certain que 〈al au(l)〉 est également présent en domaine d'oïl, mais dans une mesure bien inférieure, avec 10 à 30\%. L'action conjointe entre (i) la forte présence de ‘alı en français par des formations françaises et (semi-)savantes et (ii) le résultat héréditaire en francoprovençal conduit à une percée tout-à-fait inattendue de la notation «al aulı. Le contraste est d'autant plus flagrant avec Fribourg, qui suit, quant à elle, pleinement la logique des régions d'oïl sud-orientales.

Si nous avions hésité dans un premier temps à inclure ce paramètre dans notre analyse, nous croyons néanmoins qu'il apporte, avec toute la prudence nécessaire, un nouvel aspect qui complexifie le scénario scriptologique à l'étude. 


\section{Résultats scriptologiques, chronologiques, géolinguistiques et de phonétique historique}

Les résultats obtenus pour les trois principales issues de 'A[ non précédé de palatale, 〈a〉, 〈ei $\rangle$ et 〈é〉 permettent des interprétations plus générales qui reprennent en partie les observations que nous avons pu faire auparavant en les plaçant dans un cadre plus large.

\section{1 〈a〉}

Les graphies en 〈a〉 reflètent de toute évidence le maintien phonique de [a] < 'A[ caractéristique du francoprovençal - et de l'occitan - au sein de la Galloromania. Nous n'avons presque jamais relevé de cas où le graphème 〈a〉 se trouvait en contradiction avec la prononciation autochtone connue, ${ }^{70}$ et cette graphie est présente dans tous les territoires où l'on parlait francoprovençal. Il s'agit donc bien d'un marquage langagier oral consciemment utilisé par les scribes. Si ce premier constat peut paraître banal, il nous semble néanmoins important de rester attentif à tout instant au degré de correspondance entre les graphèmes et la langue parlée. ${ }^{71}$ Retenons que dans le cas présent, il y a pleinement cohérence entre graphie et phonie.

Le deuxième constat est que la présence de ce marquage 〈a〉 dans les documents dépend non seulement de la région en question (et donc de sa tradition scripturale) mais aussi de l'environnement phonétique et morphologique immédiat des lexèmes concernés. Cela ressort de la synthèse suivante qui réunit les pourcentages de notations de 〈a〉 dans les configurations étudiées (les pourcentages sont réalisés par rapport aux autres issues «ei é〉 etc.). Nous avons extrait cette synthèse des relevés détaillés antérieurs, en nous concentrant sur les seuls territoires francoprovençaux. Nous avons réuni les issues 〈al〉 et 〈aul〉 de QUALE / TALE en omettant le type -ALE d'origine nominale ou adjectivale puisque ses issues

70 Cf. supra 5.1, 5.2 et n. 64 la discussion détaillée des différentes occurrences : il s'agissait presque toujours de documents rédigés dans une scripta francoprovençalisante ( $\mathrm{chJu}{ }^{\circ} 13, \mathrm{chSL}$ $\mathrm{n}^{\circ} 33$ ) ou bien de documents impliquant d'une manière ou d'une autre le territoire francoprovençal.

71 Dans cette optique, nous avons également pu relever que les graphies du type iié> dans des lexèmes où -ATU n'était étymologiquement pas précédé de palatale, [j i] correspondaient pour l'essentiel à des évolutions phonétiques dans les dialectes concernés (cf. supra 5.1). 
ne représentent pas de manière univoque un marquage francoprovençal (cf. supra).

\begin{tabular}{|c|c|c|c|c|c|}
\hline Corpus & $\begin{array}{l}\text {-ATU } \\
\text { 〈a }\end{array}$ & $\begin{array}{c}\text {-ATA } \\
\langle a \text { aa ae } \\
\text { «as az ais aes } \\
\text { ayes }\end{array}$ & $\begin{array}{l}\text {-ARE } \\
\langle\text { ar }\end{array}$ & $\begin{array}{l}\text {-ATRE } \\
\langle a r\rangle\end{array}$ & $\begin{array}{c}\text { QUALE TALE } \\
\text { 〈qual quauls }\end{array}$ \\
\hline Codi & 371 [99\%] & 165 [100\%] & 474 [97\%] & 25 [86\%] & 180 [100\%] \\
\hline Grenoble & 238 [99\%] & 421 [100\%] & 84 [97\%] & 7 [100\%] & 22 [100\%] \\
\hline Lyonnais & 1397 [90\%] & 278 [89\%] & 687 [96\%] & 89 [86\%] & 403 [83\%] \\
\hline Ain & 227 [66\%] & 96 [100\%] & 32 [71\%] & 48 [84\%] & 80 [79\%] \\
\hline Forez & 706 [64\%] & $361[62,5 \%]$ & $95[41,5 \%]$ & 36 [90\%] & 14 [29\%] \\
\hline Fribourg & 165 [12\%] & $206[11,5 \%]$ & 249 [23\%] & 16 [24\%] & $10[2 \%]$ \\
\hline Vaud/Genève & 73 [12\%] & $15[7,5 \%]$ & $3[2 \%]$ & $4[7,5 \%]$ & 22 [10\%] \\
\hline $\begin{array}{l}\text { Neuchâtel } \\
\text { [NCh] }\end{array}$ & 102 [15\%] & 4 [2\%] & 0 & 0 & $15[8 \%]$ \\
\hline $\begin{array}{l}\text { total des } \\
\text { occurrences }\end{array}$ & 3281 & 1546 & 1625 & 225 & 566 \\
\hline
\end{tabular}

Ce tableau permet de cerner de manière immédiate les différences entre l'écrit du territoire francoprovençal de France et de Suisse par rapport à l'oralité francoprovençale. L'interprétation adéquate de ces issues suppose néanmoins la prise en considération du paramètre diachronique. Nous avons en effet renoncé jusqu'ici à inscrire nos observations dans une clé évolutive pour ne pas compliquer ultérieurement l'argumentation et pour pouvoir établir les différences macroscopiques à l'intérieur de l'espace francoprovençal. Les fourchettes chronologiques permettent toutefois d'accentuer très nettement nos résultats.

Commençons avec Lyon qui montre une rupture assez nette entre 1350 et 1385, autour d'une charnière qui se place vers 1365. Cela précise les observations de Pierre Gardette et Paulette Durdilly qui plaçaient déjà l'affaiblissement des issues francoprovençales vers 1350 ou 1360 (cf. supra n. 5). Voici les pourcentages de la marque 〈a〉 (ou 〈au〉) pour les cinq paramètres réunis ici : 


\begin{tabular}{lcccr}
\hline -ATU & $1225-1364$ & $95 \%$ & $1375-1400$ & $70 \%$ \\
\hline -ATA & $1305-1385$ & $95 \%$ & $1386-1389$ & $86 \%$ \\
\hline -ARE & $1250-1350$ & $100 \%$ & $1351-1425$ & $71 \%$ \\
\hline -ATRE & $1250-1350$ & $100 \%$ & $1351-1425$ & $63 \%$ \\
\hline QUALE / TALE & $1301-1363$ & $100 \%$ & $1365-1399$ & $23 \%$ \\
\hline
\end{tabular}

Il devient apparent que le marquage francoprovençal est pratiquement absolu à Lyon jusqu'en 1350. Ensuite, il s'affaiblit, d'abord pour -ARE et -ATRE (1351), puis pour QUALE/TALE (1365) 72 et pour -ATU (1375), enfin pour -ATA (1386), qui reste stable plus longtemps à cause de la juxtaposition puis la fusion des issues de $\mathrm{A}$ [ et de -A final inaccentué ([-'aa] > [-'a]). Les pourcentages baissent ainsi de 95/ $100 \%$ à environ deux tiers et à seulement un quart pour 〈qua(u)lı. La pression de la norme parisienne, qui a mené à une dérégionalisation des scriptae de Lorraine, de Bourgogne et de Franche-Comté au cours de la deuxième moitié du $14^{\mathrm{e}}$ siècle (Glessgen 2017, 338) atteint aussi Lyon, autour de la même charnière chronologique évoquée précédemment, soit vers 1360.

Pour l'Ain, il est plus difficile de se prononcer parce que le corpus de Philipon se tarit en 1365 , ce qui s'explique sans doute par le fait que les documents postérieurs étaient moins marqués et donc moins intéressants dans l'optique de l'éditeur. Un document isolé de 1465 s'oppose toutefois avec 21\% de formes en 〈a〉 pour -ATU au pourcentage des documents antérieurs (1275-1365) où cette issue reste stable autour de $70 \%$.

Ce constat vaut également pour le Dauphiné (Grenoble, Somme du code) qui rend les issues francoprovençales de manière presque absolue ${ }^{73}$ mais dont les documents se placent intégralement avant 1350.

Pour le Forez, toutefois, le positionnement entre la scripta francoprovençale et oïlique peut être suivi dans le temps. Sa logique rejoint celle de Lyon, mais elle est bien plus extrême. Voici les pourcentages de 〈a〉 (ou 〈au〉) dans leur trajectoire chronologique :

72 La charnière se trouve en 1364 où un document volumineux comporte $76 \%$ d'occurrences en 〈qua(u)l ta(u)l〉 (158 occ. de 〈al〉, 9 de 〈aul〉, 40 de «el〉 et 13 de «eil〉, par ailleurs absent de la scripta de Lyon).

73 La seule exception concerne un lexème issu de -ATRE. Nous relevons $1 \mathrm{x}$ mares et pares (Somme du Codi) ainsi que 23x frare (Somme du Code) et $7 \mathrm{x}$ à Grenoble ; mais pere se relève à 4 reprises dans la Somme du Code. 


\begin{tabular}{lcccr}
\hline -ATU & $1260-1356$ & $99,7 \%$ & $1358-1410$ & $2 \%$ \\
\hline -ATA & 1290 & $99,7 \%$ & $1358-1410$ & $2 \%$ \\
\hline -ARE & $1260-1347$ & $100 \%$ & $1358-1410$ & $55 \%$ \\
\hline -ATRE & $1288-1322$ & $100 \%$ & $1358-1422$ & 0 occ. 9$]$ \\
\hline QUALE / TALE & 1270,1290 & $74 \%$ & 1382,1410 & $0 \%$ \\
\hline
\end{tabular}

On constate donc une rupture presque totale vers 1360 quand une scripta intégralement francoprovençalisante est remplacée par une scripta pleinement oïlique. ${ }^{74}$ Le constat est net pour les 1067 occurrences des issues de -ATU/-ATA après 1356 que nous avons réunies ici. En revanche, dans le cas de «ar le pourcentage de $55 \%$ n'est pas significatif étant donné le faible nombre d'occurrences. ${ }^{75}$

Les relevés chronologiques soulignent ainsi très nettement le degré quasimaximal des marquages francoprovençaux entre l'apparition de la scripturalité vernaculaire vers 1250 et sa francisation à partir de ca 1360. L'élaboration du francoprovençal a donc été très nette, mais elle n'aura connu qu'un siècle d'existence vigoureuse.

En Suisse romande, la nature de la scripta s'avère plus faiblement francoprovençalisante. Le lieu le plus marqué dans ce sens, à savoir Fribourg, reste clairement en deçà de l'Ain, sans parler de Lyon. Les chiffres varient entre 12 et $24 \%$, et les cas de 〈qua(u)l〉 et 〈ta(u)l〉 y sont presque absents. Le pays de Vaud, Lausanne et Genève se placent avec 6 à 12\% encore à un palier inférieur, sachant qu'à l'exception de -ATU les choix ne portent pas sur les mêmes bases qu'à Fribourg. Notamment l'infinitif est pleinement oïlique, comme à Neuchâtel, selon nous toujours suite à une même volonté d'homogénéisation morphologique qu'à Lyon (ici dans la tendance opposée). À Neuchâtel, enfin, les éléments francoprovençaux se résument aux seules issues de QUALE/TALE et de -ATU avec 8 et 15\%.

Une interprétation des résultats en termes diachronique est plus délicate, justement à cause de la faiblesse des marques francoprovençales, mais aussi à cause des corpus relativement disparates en termes de lieux et de temps. Seul pour Fribourg, il est possible d'établir une chronologie large entre 1293 et 1496, au moins pour le paramètre le plus fréquent -ATU, qui est par conséquent le plus significatif. Sur ces deux siècles, il faut distinguer quatre périodes :

74 La rupture de 1358 est bien identifiée par Gonon (1974, XXI et XXIV).

75 La différence dans le nombre d'occurrences explique la forte variance des pourcentages pour le Forez dans le tableau initial de ce chapitre. 
- une première (1293-1311) dominée pleinement par l'issue oïlique non régionale 〈e〉 (35 occ. vs 1 occ. 〈ei`) : les trois documents de cette période concernant Fribourg ont en effet intégralement été rédigés en domaine d'oïl ;

- $\quad$ une deuxième période (1319-1362) comporte les débuts de la scripta francoprovençale à Fribourg avec un marquage assez net (28 occ. de «a〉, correspondant à $60 \%$ vs $36 \%$ 〈e et $4 \%$ (ei〉);

- $\quad$ une troisième période (1363-1435) voit le retrait du francoprovençal (136 occ. $=13 \%$ ) au profit de l'issue oïlique régionale «ei $>$ (598 occ. $=58 \%$, à côté de 296 occ. $=29 \%$ pour 〈e〉);

- $\quad$ enfin, la dernière période (1437-1496) pendant laquelle les issues francoprovençales disparaissent, l'issue régionale «ei〉 s'affaiblit (44 occ. $=16 \%$ ) et l'issue oïlique normative s'impose (233 occ. $=83,5 \%){ }^{76}$

Les chiffres pour les différents corpus et issues ne permettent pas de dresser un scénario précis; mais les documents de Vaud/Lausanne/Genève tout comme ceux de Neuchâtel laissent également entrevoir une première période (ca 1250 ca 1320) avec un marquage francoprovençal très faible (ca 5\%), puis une deuxième période ( $c$ a 1320 - ca 1370/1380) avec une présence plus nette des types en 〈a〉 (ca 20\%). Après, les issues francoprovençales s’affaiblissent de nouveau pour Vaud/Lausanne/Genève alors que le corpus de Neuchâtel atteint sa limite chronologique (1379).

De manière globale, l'on peut donc retenir que la présence des éléments francoprovençaux se concentre entre $c a 1310$ et $c a 1380$ avant de suivre la voie de la francisation des régions voisines. Même si cette période est plus courte qu'en France, on peut constater que les scribes des régions suisses ont développé une conscience évidente du décalage entre écrit et oral et qu'ils ont introduit un certain nombre de marques à des fins identitaires régionales. ${ }^{77}$ Il s'agit d'un phénomène circonscrit et systématiquement minoritaire par rapport aux marquages oỉliques et il porte sur un choix bien défini de types étymologiques (-ATU, pas -ARE etc.) et non sur une base phonématique générale. ${ }^{78}$

76 Notre périodisation rejoint très précisément celle proposée par Müller (2001, 247) qui retient également les charnières de 1319 («Hier erscheinen bereits ex nihilo «wohldurchdachte Graphien» [...], welche 150 Jahre lang das lokale Schrifttum bestimmen sollten ») et de 1363 (« am 31. Dezember 1363 wechselte die Gebrauchssprache dauerhaft vom Latein zum Französischen, ohne ersteres ganz verdrängen zu können »).

77 Celles-ci sont en réalité dialectales mais nous croyons que la finalité que poursuivaient les scribes devait s'inscrire davantage dans une pensée « régionale » plus de type géo-politique que linguistique.

78 Cela rejoint dans le principe même les phénomènes de conversion occitan/latin tels que nous avons pu les observer en Auvergne entre le $9^{\mathrm{e}}$ et le $11^{\mathrm{e}}$ siècle (cf. Carles 2011, § 41, 430-434) ; ceuxci portent en effet sur un nombre limité de contextes étymologiques et non sur des équivalences grapho-phonétiques généralisées (même si les deux se chevauchent partiellement). 


\section{2 〈ei>}

Le digramme «ei〉 (représentant potentiellement un type diphtongué) est pertinent autant pour la compréhension des strates linguistiques coprésentes dans les scriptae des territoires francoprovençaux que pour les trajectoires de 'A[ en français. Pour le domaine d'oïl, que nous avons interrogé à partir de notre corpus, nous formulons deux constats :

(1) Le marquage 〈ei〉 est absent seulement de la scripta parisienne (les 9 occurrences pour -ATU proviennent d'actes royaux picardisants, cf. supra n. 41) alors qu'il est présent dans une mesure variable dans l'intégralité des scriptae septentrionales, orientales et sud-orientales. Sa densité est maximale au $13^{\mathrm{e}}$ siècle en Champagne et en Lorraine ; elle est faible aux alentours de 1300 dans le SudEst oïlique. L'étendue de cette diffusion peut surprendre surtout quand on considère le fait que ce digramme (et par ricochet son interprétation phonétique) est le grand absent du traitement de 'A[ dans les manuels de référence de phonétique historique du français (à l'exception toutefois de Fouché 1952, 263s.). Seul l'article de Gossen (1968) ${ }^{79}$ examine ce digramme avec attention afin de souligner «la difficulté d'établir les équivalences entre les graphèmes et les phonèmes » (ib., 1). Nous y reviendrons.

(2) Le marquage de «ei〉 (au détriment de 〈a〉 et 〈e〉) dépend très fortement de la base étymologique des lexèmes. S'il est bien identifiable partout pour -ATU, sa fréquence relative baisse notablement pour les autres types à l'Est et dans le Nord et il disparaît presque intégralement dans le Sud-Est. Dans le tableau suivant, nous avons organisé les bases dans l'ordre de cette fréquence relative :

\begin{tabular}{|c|c|c|c|c|c|}
\hline Corpus & $\begin{array}{c}\text {-ATU } \\
\text { 〈ei eit) [<ieis] }\end{array}$ & $\begin{array}{l}\text { QUALE TALE } \\
\text { «queil teil» }\end{array}$ & $\begin{array}{c}\text {-ARE } \\
\langle e i r \sim \text { eyr }\end{array}$ & $\begin{array}{l}\text {-ATRE } \\
\text { 〈eir air> }\end{array}$ & $\begin{array}{l}\text {-ATA } \\
\text { 〈eie }\end{array}$ \\
\hline frpr. en France & 16 [0,5\%] & $21[2,5 \%]$ & $7[0,5 \%]$ & 0 & 0 \\
\hline Fribourg & 645 [46\%] & 23 [4\%] & $451[41,5 \%]$ & $4[6 \%]$ & 0 \\
\hline Vaud/Genève & 114 [18\%] & 24 [11\%] & $23[16 \%]$ & $6[11 \%]$ & 1 \\
\hline $\begin{array}{l}\text { Neuchâtel } \\
\text { [NCh] }\end{array}$ & 77 [11\%] & $8[4 \%]$ & $1[1 \%]$ & $3[4 \%]$ & 0 \\
\hline
\end{tabular}

79 Il ne nous paraît pas anodin que l'approche du seul chercheur à avoir mis en évidence ce digramme soit scriptologique et que nous prenions appui comme il l'a fait sur des sources documentaires. 


\begin{tabular}{|c|c|c|c|c|c|}
\hline Corpus & $\begin{array}{c}\text {-ATU } \\
\langle\text { «ei eit }\rangle[\langle i e i\rangle]\end{array}$ & $\begin{array}{l}\text { QUALE TALE } \\
\text { «queil teils }\end{array}$ & $\begin{array}{c}\text {-ARE } \\
\langle\text { eir } \sim \text { eyr }\rangle\end{array}$ & $\begin{array}{l}\text {-ATRE } \\
\text { 〈eir air〉 }\end{array}$ & $\begin{array}{l}\text {-ATA } \\
\text { 〈eie〉 }\end{array}$ \\
\hline $\begin{array}{l}\text { Fr-Comté, } \\
\text { Bourgogne }\end{array}$ & 283 [7,5\%] & 20 [2\%] & 9 [1\%] & 19 [3\%] & $18[1,5 \%]$ \\
\hline $\begin{array}{l}\text { Doubs [NCh], } \\
\text { Jura }[\mathrm{CH}]\end{array}$ & $1438[37,5 \%]$ & $6[0,5 \%]$ & 1 & 1 & 0 \\
\hline $\begin{array}{l}\text { Champagne, } \\
\text { Lorraine }\end{array}$ & 2246 [57\%] & $257[29,5 \%]$ & $329[30 \%]$ & $247[24 \%]$ & 127 [11\%] \\
\hline Douai, Marne & $651[36,5 \%]$ & 82 [18\%] & 44 [7\%] & $24[5 \%]$ & 14 [2\%] \\
\hline ChRoy & 9 & 0 & 0 & 0 & 0 \\
\hline total & 5479 & 349 & 865 & 304 & 160 \\
\hline
\end{tabular}

En ligne générale, la distribution observable semble indiquer une tendance évolutive à l'affaiblissement voire à la disparition du digraphe. Il est légitime de supposer que l'écrit reflète ici une tendance de l'oral, où l'affaiblissement d'une issue héréditaire voire une nouvelle variante de celle-ci se diffusent très concrètement en fonction des environnements phonétiques et des fonctions morphologiques précises. En même temps, l'écrit accentue sans doute le scénario puisque, comme nous l'avons déjà vu, les scribes choisissent facilement un marquage bien défini pour conférer une touche régionale à leur scripta. Dans le cas présent, ce choix porte sur les issues de -ATU, facilement reconnaissables et peut-être même plus nettes aussi à l'oral. La faiblesse particulière pour -ATA s'explique, quant à elle, assez bien par la réticence évoquée à l'égard des trigrammes et triphtongues.

Pour le domaine francoprovençal, c'est surtout l'opposition entre la Suisse et la France qui ressort de ce tableau synthétique. Les scriptae régionales et locales de la Suisse s'alignent très nettement vers les scriptae oïliques du Sud-Est alors que les scriptae autour de Lyon et du Dauphiné ne suivent aucunement ces tendances. Comme nous l'avons déjà formulé, dans leur part oïlique, ils s'orientent clairement vers Paris et la région autour de la capitale.

Dans le détail, chaque ensemble suisse fait apparaître des choix propres, ce qui souligne la relative indépendance de ces lieux d'écriture entre eux : le pays de Vaud, Lausanne et Genève rend les issues de 'A[ avec une homogénéité qui n'est observable nulle part ailleurs : à l'exception de «eies, les quatre autres ensembles se placent tous entre 11 et $18 \%$. Neuchâtel suit en revanche plus étroitement la logique du Sud-Est oïlique avec un marquage net de -ATU et un marquage faible pour les autres ensembles. Fribourg enfin surprend, comme nous l'avons déjà vu, avec le fort marquage non seulement de -ATU, mais aussi de -ARE. 
En synthèse, les scriptae francoprovençales se distinguent donc par trois aspects entre les territoires de la Suisse et de la France actuelles :

- en Suisse elles sont (i) moins marquées du point de vue du francoprovençal, mais (ii) régionalisées du point de vue du français et (iii) assez divergentes entre elles ;

- en France elles sont (i) beaucoup plus nettes quant au francoprovençal, mais (ii) dérégionalisées quant au français et (iii) plus homogènes.

Nous sommes en face des effets d'une véritable élaboration scripturale de cette langue à Lyon et dans le Dauphiné, alors qu'en Suisse, il s'agit plutôt d'une strate de l'oral qui s'insère de manière plus aléatoire dans un modèle scriptologique introduit de l'extérieur.

\section{3 〈ei és}

Jusqu'ici, nous avons considéré le digramme eì dans son rôle de marqueur régional. Son interprétation implique toutefois aussi une réflexion concernant l'évolution phonétique et géolinguistique de 'A[ en français. Voici la synthèse formulée par l'un d'entre nous il y a dix ans (Glessgen 2008, 473) :

« Genèse : la diphtongaison de /a / accentué en syllabe ouverte (lat. /'a:/ /_\$ > /e / 〈ei) est considérée parfois comme une évolution ultérieure de /e:/ (< lat. /'a:/ /_\$), parfois comme un reliquat de l'ancienne diphtongaison qui a mené de /a:/ à /e:/ (a $>{ }^{\star} a e>^{\star} \varepsilon$ : $>$ ee > e: , cf. Gossen 1967, 140 ; 155-157)».

Nous pensons aujourd'hui que les deux interprétations sont justes, selon les régions concernées. Il est en effet très probable qu'à l'Ouest, [ei] soit une évolution secondaire par rapport à la monophtongue [' $\varepsilon: \sim$ 'e:], ${ }^{80}$ alors que dans le Nord-Est, il pourrait bien s'agir d'une variante du stade *['عe], donc d'une évolution parallèle sinon antérieure à la monophtongue.

La question mérite l'attention parce que la bibliographie ne prend que peu en considération le type «eì. Celui-ci est bien moins apparent dans les textes littéraires - qui restent encore à l'heure actuelle le point d'appui des travaux sur l'ancienne langue - que dans les actes originaux ou encore dans les dialectes

80 C'est aussi l'interprétation la plus plausible des données réunies par Brasseur (2018) pour la Normandie où des formes diphtongués du type [عi] (<-ARE) ou, le plus souvent, [ai $\sim$ bi $\sim$ a $\varepsilon$ ] font surface en divers lieux (cf. ib., 96s., 102s., 107s.). 
modernes.$^{81}$ C'est ainsi que le traitement le plus approfondi se trouve dans les travaux de Gossen (1967, 139-157 et 1968, 10-16) qui, toutefois, en donne une interprétation trop partielle et trop réduite. Selon lui, le type «ei est partout secondaire par rapport à 〈e〉 et fait apparition dans les régions périphériques (Nord-Ouest et Nord-Est), avec une dynamique d'extension vers le centre du domaine (cf. la carte 1968, 13). Gossen met également en cause l'éventuelle réalisation du digramme comme diphtongue. ${ }^{82}$

Nous croyons cette idée incohérente d'un point de vue géolinguistique. «ei〉 est en effet présent - pour des raisons différentes - dans les scriptae documentaires de Normandie, de Picardie, de Wallonie, de Champagne et de Lorraine, et ceci au moins depuis le $11^{\mathrm{e}}$ siècle..$^{83}$ Elle apparait dans une moindre mesure dans celles, plus tardives, du Sud-Est. Le type «è en revanche est exclusif à Paris dans la $2^{\mathrm{e}}$

81 Rappelons que les textes littéraires nous sont transmis pour la très grande majorité d'entre eux sous la forme de copies plus ou moins postérieures aux œuvres initiales et plus ou moins influencées - à partir de ca 1330 - par la norme de la chancellerie royale (cf. Glessgen 2017). Par ailleurs, nous savons que les copies réduisent de manière intrinsèque les marques dialectales de l'oral (cf. ib., 327 n. 35). La combinaison des différents paramètres peut avoir l'effet macroscopique qu'un type graphématique donné ne fait pas pleinement apparition dans ces témoins textuels.

82 Gossen (1968, 14 ; cf. aussi 1967, 151) avance en effet, l'argument que la graphie «i pourrait ne pas marquer un deuxième élément de diphtongue mais pourrait être un signe d'allongement vocalique. Pour examiner la validité de cette hypothèse, nous avons relevé avec beaucoup d'attention les occurrences de «ì qui pouvaient s'inscrire dans la logique d'un tel $i$-'parasite' dans nos corpus. Pour la Meurthe-et-Moselle nous avions identifié, dans un souci d'exhaustivité, 122 occurrences de «ei ou de «aì ne notant pas une diphtongue (beites, juleit, eiglise, frerei, faimme, aiquest etc., Glessgen 2008, 479s.) contre 1187 occurrences de sei qui correspondent à 'A[ latin (ib., 477s.). Étant donné que ce digramme connaît non moins de 7000 occurrences dans notre corpus intégral actuel, toujours comme issue de 'A[, que les formes avec un «i-parasite» restent assez rares en dehors de la Lorraine et que les formes dialectales modernes confirment clairement l'idée d'une diphtongue, l'argument de Gossen semble peu probant. On a pu en outre observer dans une autre étude que le graphème 〈i connaît de nombreuses valeurs phoniques mais aussi que les scribes tendent à l'utiliser quand ils sont confrontés à devoir noter des sons ressentis comme difficiles à mettre à l'écrit (cf. pour la mise en évidence du phénomène et des exemples de notations de phonèmes consonantiques affriqués en domaine occitan, Carles 2011, 397ss. $§ 6$ et 7 avec renvois). Il n'est donc pas incongru de supposer que le graphème puisse avoir une valeur différente dans frerei et dans freire.

83 Cf. aussi dans le Trésor galloroman des origines pour -ATu les toponymes anorm. la Ferteit (11 ${ }^{\mathrm{e}}$ s.) à côté de la Fertet (1046/48, Carles 2017 s.v.), alorr. Grant Preit (1117, Toul, s.v. albue); pour -ATA non précédé de palatale aang. lat. NL la Preia (1104, Marmoutier, s.v.) à côté de la Pree (ca 1094) et les lexèmes aang. lat. rameia (1104/1124, Marmoutier, s.v.) et turseia (1067/1100, Bourgueil, s.v. [la base étymologique est indéterminée, mais l'affixe est transparent]) ainsi que - à Paris (!) - corveia (ca 1093, s.v.); s'ajoutent après palatale - où notre corpus connaît le type seulement pour la Meuse - aang. lat. tal(l)eia (1101/1103, Marmoutier, s.v.) et apoit. lat. cava[l]cheia (1119/1120, s.v.). 
moitié du $13^{\mathrm{e}}$ siècle - quand les premiers textes apparaissent - et aussi dans le pays de la Loire. Le type phonétique [ei], quant à lui, survit réellement dans les dialectes de toutes les régions du «ei〉 médiéval comme variante mineure (cf. FEW S.v. PRATU). La simple distribution dans l'espace plaide donc ici pour un type grapho-phonétique ancien remplacé par une innovation venant du centre du domaine d'oîl, innovation qui s'est répandue dans l'espace à travers les siècles. À l'écrit, le type «e`s'impose encore bien plus nettement à partir de ca 1330 avec la diffusion de la norme scripturale parisienne. Les formes de l'écrit documentaire en 〈ei〉 et les formes dialectales modernes de [ei] s'inscrivent plutôt dans un scénario de la Linguistica areale de Bartoli et les données de nos corpus laissent entrevoir assez nettement cette tendance en cours qui mène à l'affaiblissement du type «eì.

Toutefois, les évolutions comprises entre le $6^{\mathrm{e}}$ et le $12^{\mathrm{e}}$ siècles restent largement opaques. L'on suppose généralement, avec quelques variations selon les

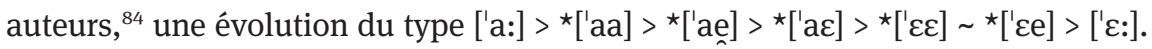
L'étape [' $\varepsilon:]$ devait déjà être atteinte aux $7^{\mathrm{e}} / 8^{\mathrm{e}}$ siècles. ${ }^{85}$ L'ancienneté de ce résultat et la complexité de l'évolution, fortement dépendante de ses environnements, ${ }^{86}$ laissent une marge importante de coexistence avec des variantes à différents moments. Le digramme «ei des $11^{\mathrm{e}} / 13^{\mathrm{e}}$ siècles pourrait être un reflet, au moins

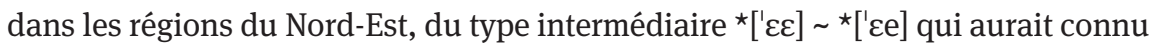
une existence parallèle à [' $\varepsilon$ : 'e:]. Après l'évolution de [ei] (provenant de 'E[ latin) en [oi] etc., la diphtongue ${ }^{\star}\left[{ }^{\prime} \varepsilon \varepsilon\right] \sim{ }^{\star}[$ 'ce] a pu évoluer ultérieurement vers [ei]. Comme Jean-Paul Chauveau nous l'a fait remarquer, "les aboutissants de PATRE et PIRA ont pu s'écrire «peire` tous les deux, mais ils n’ont jamais été homophones $»{ }^{87}$

84 Cf. Fouché (1952, 263s.), Bourciez/Bourciez (1967, § 35, 55-57), Straka (1979, 190s.) [= 1959, ZrP 12, 300], La Chaussée (1989, 108ss.), Zink (1991, 56s.), Pierret (1994, § 426s.), Englebert (2009, 54s.).

85 Cf. l'attestation préda de-pascis < PRATA 727 dans les chartes mérovingiennes (Vielliard 1927,

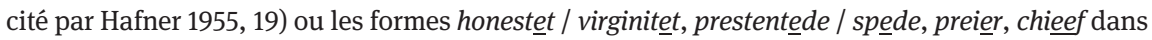
la Séquence de Sainte Eulalie (alors que les Serments de Strasbourg, plus latinisants, gardent 〈a).

86 L'on sait notamment que devant nasale, l'évolution de 'A[ est retardée. Ici, J.-M. Pierret

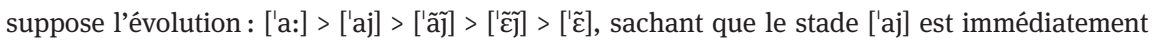
reflété par la graphie traditionnelle «ai〉, qui a survécu jusqu'aujourd'hui.

87 Lettre du 10 févr. 2018. - Notons que notre explication rejoint aussi l'évolution de 'A[ devant un son palatal (palatiu, Gladiu, Plaga etc.). Pierret (1994, § 453) retient l'évolution : ['a:] > ['ai] > ['عi] (vers 1100) > [' $\varepsilon$ :] (12 $/ 17^{\mathrm{e}} \mathrm{s}$.). Ici l'état roman initial ['ai] se fond avec [i] étymologique suivant, ce qui retarde les évolutions postérieures, tout comme devant nasale (cf. Pierret, ib. : ['ain] assonne encore en $[\mathrm{a}]$ au $11^{\mathrm{e}} \mathrm{s}$.). Mais la trajectoire générale est identique, ce qui rend l'évolution ['ai] > ['عi] plus plausible. 
Ajoutons deux observations concernant la première phase de l'écrit oïlique :

(1) La graphie 〈maent < 'MAN(E)T dans la Séquence de Sainte Eulalie pourrait être prise comme un indice d'un état intermédiaire *['ã $\tilde{\varepsilon}]$, parallèle à *['ac], ou éventuellement une diphtongue de type ['ã̃]].

(2) Plus intéressant : l'absence de «ei〉 dans les Serments de Strasbourg et, surtout, dans la Séquence de Sainte Eulalie (provenant d'un territoire qui devait connaître un type *['ce] à l'oral) rejoint, renforce et accentue dans un sens la thèse de Gerold Hilty qui suppose - grâce à la présence de la consonne épenthétique type 〈ldr ndr (voldret, voldrent, sostendreiet) - une influence de la région du Centre (Hilty 1993, 12-15). De manière plus neutre, nous supposons qu'il existait dès la première époque un dialogue scriptural entre les régions, dialogue qui passait par le canal de la mise à l'écrit des formes vernaculaires en contexte latin (Carles 2011). Ce dialogue pouvait mener à des interférences et à des emprunts entre une région et une autre, cohérente avec la démarche supra-régionale des premiers textes vernaculaires.

Indépendamment de ces questions de genèse, le type [' $\varepsilon \sim$ 'e] 〈e〉 semble bien être au $13^{\mathrm{e}}$ siècle une variante régionale du centre du domaine d'oïl autrement dit de Paris. Ce serait donc un des rares régionalismes phonétiques (et graphématiques) qui caractérisent la langue et la scripta de la capitale et qui l'opposent à l'essentiel du territoire d'oïl.

\section{7 Étude complémentaire : lat. 'A[ après palatale et après / $\mathbf{i} \mathbf{j} /$}

Les issues de 'A[ non précédé de palatale permettent d'identifier une partie importante des tendances qui déterminent la physionomie et l'évolutivité des scriptae entre le français et le francoprovençal. Notre traitement détaillé a par ailleurs pu illustrer autant la nature de nos données que nos choix et approches méthodologiques.

Pour compléter le raisonnement précédemment mené, nous synthétiserons par la suite nos résultats concernant les issues après palatale. Le rendement pour les scriptae francoprovençales est ici mineur puisque le francoprovençal partage dans un premier temps l'issue ['jc] avec le français et ne s'en détache qu'ensuite en passant à ['ja], en rejoignant les issues de 'A[ non précédé de palatale. ${ }^{88}$ Mais

88 Hafner $(1955,64)$ suppose que cette évolution ultérieure s'est produite au courant du $13^{\mathrm{e}}$ siècle; d'après nos données, l'issue phonétique en ['ia] est pleinement atteinte dès le milieu du 
autant la complexité des issues après consonne ou groupe palatal que la divergence des issues entre -ATU / -ARE d'une part et -ATA d'autre part ajoutent des aspects complémentaires aux observations que nous avons déjà pu faire.

\section{1 -ATU après palatale et / $\mathrm{i} j /(7580$ occ.)}

Une fois opérées les clarifications préliminaires (cf. supra 4.3), nous avons pu structurer les quelque 7500 occurrences concernées pour -ATU. Le paramètre est difficile à appréhender et nous avons distingué non moins de dix résultats graphématiques pour les issues après un groupe palatal et sept notations après $/ \mathrm{i} j /$. Certaines sont très peu fréquentes (notamment les variantes avec un 〈-t $\rangle$ graphique ou avec le digramme (ii), mais la répartition est néanmoins significative et demande une hiérarchisation cohérente :

- après palatale et après /i $\mathrm{j} /$ apparaissent les quatre types principaux «a ei é $\sim$ iés, accompagnés pour la plupart de variantes avec un 〈-t〉 graphique : 〈a〉

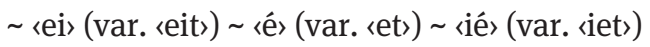

- seulement après palatale se mettent en place les types <ia $\sim$ iei comportant comme premier élément graphique 〈i : $\langle$ ia $\sim\langle$ iei (var. 〈iiei $\sim$ ieit $\rangle$ )

- La précession de / $\mathrm{j} /$ dans le radical mène parfois au digramme <ii, peu apprécié par les scribes. ${ }^{89}$ Les variantes ¿iiei ieit», quant à elles, restent très rares (1 seule occurrence de «iiei en Meuse, 2 occ. de 〈ieit〉 en Meuse, 3 à Fribourg).

Nous avons regroupé dans un même tableau les résultats des deux séries :

$13^{\mathrm{e}}$ siècle puisque les types en «(i)a〉 dominent dès les premiers textes à Lyon (1250), dans le Forez (1270) et dans l'Ain (1275). Le fait que dans le Codi, proche de l'oralité francoprovençale, 〈és domine encore ( 23 occ. $v s 14$ de 〈a ) semblerait indiquer qu'au début du $13^{\mathrm{e}}$ siècle - moment de la traduction de ce texte -, le changement n'était pas encore achevé (Hafner ib. cite en effet le Codi, mais en supposant que le type «e` y était généralisé). Il faudrait donc sans doute placer le changement au courant du $12^{\mathrm{e}}$ siècle et sa généralisation vers le milieu du $13^{\mathrm{e}}$.

89 Cf. déjà Carles (2011, 400 § 7.1). 


\begin{tabular}{|c|c|c|c|c|c|c|c|c|c|c|}
\hline Corpus & $\langle a\rangle$ & 〈ia〉 & $\begin{array}{c}\text { «ei iei iiei } \\
\text { ieits } \\
\text { [reits] }\end{array}$ & 〈é et〉 & 〈ié iet〉 & total & $\langle i\rangle+\langle a\rangle$ & $\begin{array}{l}\langle\mathrm{i}\rangle+\langle\mathrm{ei}\rangle \\
\text { [〈eit }\rangle]\end{array}$ & $\begin{array}{c}\langle\mathrm{i}\rangle+\langle e ́ \text { ié } \\
\text { et iet〉 }\end{array}$ & total \\
\hline Codi & 14 & 0 & 0 & 23 & 7 & 44 & 1 & 0 & 0 & 1 \\
\hline Grenoble & 0 & 0 & 0 & 1 & 1 & 2 & 3 & 0 & 675 & 678 \\
\hline Lyonnais & 3 & 102 & 0 & 27 & 28 & 160 & 1072 & 0 & 21 & 1093 \\
\hline Ain & 1 & 21 & 0 & 8 & 9 & 39 & 5 & 0 & 69 & 74 \\
\hline Forez & 15 & 2 & 0 & 78 & 20 & 115 & 3 & 0 & 613 & 616 \\
\hline $\begin{array}{l}\text { frpr. en } \\
\text { Suisse }\end{array}$ & 2 & 0 & 14 & 45 & 211 & 272 & 1 & 0 & 480 & 481 \\
\hline $\begin{array}{l}\text { Fr-Comté, } \\
\text { Bour- } \\
\text { gogne }\end{array}$ & 2 & 0 & 14 & 177 & 849 & 1042 & 0 & 8 & 763 & 771 \\
\hline $\begin{array}{l}\text { Cham- } \\
\text { pagne, } \\
\text { Lorraine }\end{array}$ & 0 & 0 & 47 & 54 & 1136 & 1237 & 0 & 93 & 375 & 468 \\
\hline $\begin{array}{l}\text { ChRoy, } \\
\text { Douai }\end{array}$ & 0 & 0 & 0 & 28 & 327 & 355 & 0 & 0 & 132 & 132 \\
\hline total & 37 & 125 & 75 & 441 & 2588 & 3266 & 1085 & 101 & 3128 & 4314 \\
\hline
\end{tabular}

Voici les différents constats :

(1) 〈ié〉 : cette issue connaît une diffusion homogène, indifféremment de son origine étymologique : le digramme peut reposer sur pal+ ${ }^{A}$ [ ou sur $/ \mathrm{i} j /+{ }^{\mathrm{A}} \mathrm{C}$. La notation attendue en domaine d'oill reflétant l'effet de Bartsch en ['je] est largement dominante, aussi sur le territoire francoprovençal, à l'exception de Lyon et $\mathrm{du}$ Codi ainsi que de l'Ain (au moins après palatale);

(2) 〈é̀: après palatale, 〈ié〉 est concurrencée, essentiellement en domaine francoprovençal et dans le Sud-Est oïlique, par 〈é» (donc sans marquage de la semi-consonne); cette concurrence se concentre sur un nombre défini de lexèmes : surtout assigné et - dans le Codi - comencé, ponctuellement ligé, obligé, partagé, touché, laissé et renoncé;

(3) 〈ei : le type oïlique minoritaire «ei〉 est surtout présent en Champagne et en Lorraine, faiblement après palatale (4\%), plus nettement après $/ \mathrm{i} \mathrm{j} /(20 \%)$; après palatale, il apparaît également dans le Sud-Est oïlique et sur le territoire francoprovençal de la Suisse ; la marque régionale rejoint donc ici, dans une mesure affaiblie, les tendances des issues non précédées de palatale ; 
(4) 〈(i)a〉: le type francoprovençal est pratiquement absent du territoire francoprovençal de la Suisse, contrairement à la France. Après / i j/ «a〉 est généralisé à Lyon (98\%), mais presque absent ailleurs. Après palatale, «(i)a reste majoritaire à Lyon jusqu'en 1378 (65\% : 86 occ. en «(i)a〉 vs 47 en «é et ié〉 entre 1250 et 1378) et devient minoritaire par la suite (32\% : 19 occ. en «(i)a〉 vs 40 en «é et ié` entre 1384 et 1399) ; le type est également majoritaire dans l'Ain (56\%) - où les documents se tarissent en 1365 - et présent dans le Codi (32\%) ; dans le Forez «(i)a〉 est généralisé dans un couple d'actes de 1270, mais absent des autres occurrences qui se placent toutes après 1358 .

Le marquage francoprovençal après palatale reste donc assez fort en France jusqu'en ca 1360/80. Les issues 〈ia〉 et 〈a〉 connaissent d'ailleurs une distribution complémentaire : 〈ia〉 à Lyon et dans l'Ain, 〈a〉 à Forez et dans le Codi ;

(5) 〈-t〉: notons par ailleurs que les variantes comportant un élément palatal suivi d'un 〈-t〉 graphique sont de nouveau caractéristiques de Douai et ponctuellement présentes surtout en Lorraine :

Douai : 〈eit 79x vs «ei 7 $7 \mathrm{x}$ / 〈iet $94 \mathrm{x} v s$ 〈ié> 59x / 〈iiet 20x vs 〈iié> 19x

par ailleurs : «eit〉 Meuse 1x, MMoselle 2x, Lyon 1x, <ieit MMoselle 2x, <iet Marne 15x, Meuse 8x, MMoselle 1x, HMarne 1x, HSaône 5x, JurBern 1x, «iiet> Nièvre 1x, JurBern 1x. enfin : le type «iet〉 est curieusement fort à Lyon ( 23 occ. contre 35 occ. de 〈ié)).

(6) 〈iié $\sim$ iiet $\sim$ iiei〉: les trigrammes 〈iié $\sim$ iiet〉 sont caractéristiques de Douai (39x 〈iié iiet〉 vs 153x 〈ié iet) ) et de Porrentruy ( $86 \mathrm{x}$ 〈iié〉 vs 334x 〈ié〉) ; ailleurs ils sont rares (7x Neuchâtel, 5x Marne et HMarne, 3x Côte-d'Or, 2x Meuse et MMoselle, 1x Vosges, HSaône et Jura, Vaud/Genève et Forez - en tout 29 occurrences contre plus de 3000 sans dédoublement graphique de 〈i〉). La forme 〈iiei est, quant à elle, très isolée (Meuse $1 \mathrm{x}$ vs $6 \mathrm{x}$ 〈iei ).

En synthèse, les issues qui s'éloignent du type iée sont nettement moins fréquentes que pour 'A[ non précédé de palatale, tout en s'inscrivant dans les mêmes logiques géolinguistiques. Nous avons à nouveau constaté la faiblesse des trigrammes. On retiendra l'absence presque totale des issues francoprovençales en Suisse qui s'oppose de nouveau à la France, ou le type «(i)a〉 est bien présent après palatale, au moins jusqu'en 1360/1380. La position de Lyon est surprenante, puisque cette région choisit, contrairement à tout le reste du territoire francoprovençal, de généraliser la forme innovatrice «(i)a〉 après /i j/. Le rendement de ce paramètre pour notre interrogation francoprovençale est donc circonscrit, mais réel. 


\section{2 -ARE après palatale ou après / $\mathrm{i}$ j/ (2678 occ.)}

La distribution des issues de -ARE est assez semblables à celle de -ATU. Nous présentons par la suite les résultats sous forme de tableau - en réunissant tous les corpus oïliques - et nous formulerons par la suite quelques observations.

\begin{tabular}{|c|c|c|c|c|c|c|c|c|c|c|}
\hline Corpus & ar & iar & «eirs & $\langle e r\rangle$ & 〈ier $\rangle$ & $\langle\mathrm{i}\rangle+\langle a r\rangle$ & $\begin{array}{l}\langle\mathrm{i}\rangle+ \\
\langle\mathrm{eir}\rangle\end{array}$ & $\langle\mathrm{i}\rangle+\langle e r\rangle$ & $\langle\mathrm{i}\rangle+\langle$ ier $\rangle$ & total \\
\hline Codi & 0 & 0 & 0 & 30 & 0 & 2 & 0 & 63 & 0 & 95 \\
\hline Grenoble & 0 & 0 & 0 & 1 & 1 & 1 & 0 & 8 & 0 & 11 \\
\hline Lyonnais & 1 & 3 & 0 & 3 & 95 & 12 & 0 & 86 & 0 & 200 \\
\hline Ain & 0 & 0 & 0 & 0 & 27 & 0 & 0 & 24 & 0 & 51 \\
\hline Forez & 9 & 0 & 0 & 16 & 25 & 5 & 0 & 24 & 1 & 80 \\
\hline Fribourg & 0 & 0 & 4 & 6 & 111 & 6 & 4 & 96 & 0 & 227 \\
\hline Vaud/Genève & 0 & 0 & 2 & 1 & 48 & 0 & 0 & 36 & 2 & 89 \\
\hline Neuchâtel [NCh] & 0 & 0 & 0 & 0 & 25 & 0 & 0 & 32 & 0 & 57 \\
\hline domaine d'oïl & 0 & 0 & 6 & 35 & 884 & 1 & 9 & 857 & 85 & 1868 \\
\hline total & 10 & 3 & 12 & 92 & 1231 & 26 & 13 & 1226 & 88 & 2678 \\
\hline
\end{tabular}

(1) 〈ier〉 : le type est presque généralisé en domaine d'oïl (comme 〈ié〉 de -ATU), et également dans le territoire francoprovençal de Suisse et même de France.

(2) 〈ei : ce type oïlique régional reste ponctuel, autant après palatale qu'après /i j/ (6x Meuse et MMoselle vs 115/132x «ier), 8x Fribourg vs 207x <ier), 1x Douai, Marne, HMarne).

(3) 〈(i)ar〉: le type francoprovençal «(i)ar» ne connaît une présence significative que dans le Lyonnais (16x vs 181x ier) et dans le Forez (14x vs 66x (i)er», sachant que sar se place de nouveau exclusivement en 1270); par ailleurs, il est rare (Fribourg 6x vs 207x ier), Grenoble $1 \mathrm{x}$ vs 9x «ier), Codi $2 \mathrm{x}$ vs 93x (i) er).

(4) 〈iier〉: le dédoublement graphique de 〈i (donc «-ier» après [i j] du radical) reste un choix minoritaire dans les scriptae galloromanes; on ne relève que 88 cas de ce type (contre 1226 cas de sier provenant de [i j] + A'[ ) ; ces trigrammes se concentrent à Douai (21x «ier〉 contre $162 \mathrm{x}$ (ier) ) et, de manière quelque peu surprenante, à Porrentruy (46x 〈iier〉 contre $174 \mathrm{x}$ 〈ier») ; dans les autres régions, ces formes sont rares ou absentes (en tout 15x dans Marne, Meuse, MMoselle, 
Vosges et HMarne, $1 \mathrm{x}$ dans ChRoyales, 5x dans Neuchâtel, Vaud/Genève et Forez, absentes dans les dix autres corpus).

Pour -ARE, on note donc un marquage francoprovençal encore plus faible que pour -ATU puisque même Lyon choisit pour l'essentiel les issues oïliques. Si pour -ATU, 〈(i)a〉 était plus présent après palatale, pour -ARE, c'est après /i j/ que 〈(i)ar〉 est un plus présent. Mais, ici encore, le paramètre est très peu réactif pour la strate francoprovençale dans les scriptae.

\section{3 -ATA après palatale et après / i j/ (934 occ.)}

Les issues de -ATA se placent dans la même logique que celles de -ATu, à la seule différence près qu'il manque les variantes avec un 〈-t〉 graphique qui pour -ATA n’ont de raison d'être ni étymologique ni morphologique. En voici l'inventaire :

après palatale et après $/ \mathrm{i}$ j/: 〈a $\sim\langle$ iei $>$ 〈ee $\sim$ 〈iee〉

après palatale : 〈ia〉 〈ieie〉 (var. ¿iieie〉)

En regroupant les différents corpus oïliques et en séparant les résultats après palatale de ceux après / $\mathrm{i} /$, les issues relevées sont les suivantes :

\begin{tabular}{|c|c|c|c|c|c|c|c|}
\hline Corpus & $\langle a\rangle$ & 〈ia〉 & 〈ee & $\begin{array}{c}\langle\text { iee } \\
\text { [〈ieie»] }\end{array}$ & $\langle i\rangle+\langle a\rangle$ & $\begin{array}{c}\langle\mathrm{i}\rangle+\langle e e\rangle \\
{[\langle\mathrm{i}\rangle+\langle e i e\rangle]}\end{array}$ & total \\
\hline Codi & 28 & 27 & 0 & 0 & 8 & 0 & 63 \\
\hline Grenoble & 0 & 70 & 0 & 0 & 0 & 0 & 70 \\
\hline Lyonnais & 4 & 143 & 3 & 1 & 16 & 0 & 167 \\
\hline Ain & 3 & 45 & 0 & 0 & 0 & 0 & 48 \\
\hline Forez & 0 & 181 & 14 & 4 & 0 & 3 & 202 \\
\hline Fribourg & 1 & 1 & 12 & 0 & 0 & 7 & 21 \\
\hline Vaud/Genève & 0 & 2 & 2 & 0 & 6 & 2 & 12 \\
\hline Neuchâtel [NCh] & 0 & 1 & 3 & 1 & 0 & 2 & 7 \\
\hline domaine d'oïl & 0 & 0 & 79 & 135 & 0 & 130 & 344 \\
\hline total & 36 & 470 & 113 & 141 & 30 & 144 & 934 \\
\hline
\end{tabular}


Cette présentation fait apparaître trois constats qui se placent en contraste surprenant avec les issues de -ATU et de -ARE :

(1) 〈(i)a〉 : la prédominance des issues francoprovençales est presque absolue en France, y inclus dans le Forez jusqu'aux années 1380 (le type «ee` apparaît en 1381 et se généralise entre 1389 et 1410). Ce résultat peut s'expliquer par la superposition des issues de 'A[ et de -A final inaccentué (cf. supra 5.2). En Suisse, les issues francoprovençales restent néanmoins rares.

L'on constate par ailleurs l'alternance entre 〈a〉 et 〈ia〉 dans le Codi et la coprésence de 〈a〉 et 〈ee〉 dans Vaud/Genève, toutefois avec très peu d'occurrences $(2+2)$.

(2) 〈eie〉: les issues en 〈ei〉 (concrètement 〈(i)eie») disparaissent presque intégralement, par rejet de longueur de la séquence vocalique; dans le tableau, nous avons regroupé les rares cas avec les issues voisines (uniquement dans la Meuse : $16 \mathrm{x}$ 〈ieie», $1 \mathrm{x}\langle\mathrm{i}+\mathrm{eie}\rangle$ ).

(3) 〈ee〉 : l'absence de 〈i〉 devant 〈ee〉 est presque générale en Suisse et elle est plus forte que pour -ATU en domaine d'oill. Il peut toutefois s'agir ici d'un effet optique : les chiffres sont relativement faibles, et les lexèmes concernés restent en partie les mêmes que pour -ATu (surtout assignee / designee / signee et var. ainsi que ligee, mais aussi baillee, fauc(h)ee etc.).

\subsection{Synthèse pour ' $\mathrm{A}$ [ après palatale}

Après palatale et [i j], les résultats de 'A[ sont moins nets que ceux non précédés de palatale. Les marquages autant francoprovençaux que régionaux sont moins présents, à l'exception toutefois des issues de -ATA où le type en «(i)a〉 est pratiquement généralisé en France. Ce résultat rejoint donc pleinement les résultats de -ATA non précédés de palatale.

Le paramètre reste ainsi pertinent pour suivre les éléments francoprovençaux dans les textes mais par les aléas des actions d'environnement, des choix scripturaux et, éventuellement aussi, par le fait que le résultat primitif du domaine francoprovençal est identique à celui en domaine d'oïl, il est beaucoup moins net dans la plupart de ses configurations.

\section{Interprétation des résultats}

L'analyse détaillée des issues de 'A[ a pu avérer que ce paramètre est hautement significatif pour tracer les voies générales de la réalité scripturale du francoprovençal médiéval. Elle a également pu montrer l'intérêt de cette approche qui 
réside dans la tentative d'identifier la nature et le degré de l'élaboration de cette langue romane, tombée un peu dans l'oubli en dehors des cercles scientifiques spécialisés.

Essayons de synthétiser les constats mis en avant à travers notre étude.

\subsection{Les territoires de la France et de la Suisse}

La distinction majeure, récurrente dans tous nos relevés, concerne celle entre les deux grandes régions scriptologiques du francoprovençal, d'une part Lyon avec l'Ain et le Forez ainsi que le Dauphiné, et d'autre part la Suisse, avec ses traditions souvent locales entre Fribourg, Neuchâtel et le pays de Vaud, Lausanne et Genève. L'élaboration scripturale la plus avancée du francoprovençal se place en France, où du milieu du $13^{\mathrm{e}}$ siècle jusque vers 1360 les choix grapho-phonétiques autochtones peuvent pleinement évincer les formes oïliques. En Suisse, l'élaboration écrite $d u$ francoprovençal se produit plus tardivement et pendant une période plus restreinte, de ca 1320 à ca 1380 tout en restant plus contenue : la part oïlique est presque toujours majoritaire, les issues francoprovençales sont plus variables selon les environnements phonétiques et moins homogènes selon les régions. L’analyse scriptologique illustre avec beaucoup de précision le scénario général d'une élaboration scripturale bipartite du francoprovençal et permet d'en établir une chronologie nette.

Notre analyse montre ensuite que les scriptae francoprovençales de France et de Suisse ne diffèrent pas seulement par le degré et les modalités de leur élaboration, mais aussi par les modèles oïliques qui sont impliqués. Les régions suisses se sont en effet orientées, à l'exception d'une toute première phase, vers une scripta oïlique régionale du Sud-Est, alors que les textes provenant de la région autour de Lyon sont pratiquement exempts, à tous les moments, de traits oïliques sud-orientaux.

Dans le cas de la Suisse, les scriptae de Neuchâtel et du pays de Vaud, Lausanne et Genève semblent particulièrement proches des scriptae méridionales des régions immédiatement voisines de la Franche-Comté (Jura français) et, dans une moindre mesure, de la Bourgogne (Saône-et-Loire). ${ }^{90}$ La présence du di-

90 Déjà Gossen rapproche la scripta neuchâteloise de celles « de la Bourgogne, de la FrancheComté et du Jura bernois » $(1965,456)$ : «nous assistons ici à un processus d'adaptation progressive de la part de la scripta neuch. aux normes graphiques en usage dans le sud-est du domaine d'oïl » (ib., 457); « entre la Franche-Comté, avec son centre Besançon, et Neuchâtel, d'une part, et le Jura dit bernois, de l'autre, il y avait d'innombrables occasions de contact» (ib., 458). Notons dans ce contexte que de nombreux mots oïliques régionaux du Sud-Est apparaissent dans les 
gramme «ei〉 évoque toutefois davantage les modèles de la Lorraine et de la Champagne méridionales où la scripturalité était bien plus développée vers 1300 . L'analyse d'un nombre plus important de paramètres - également lexicaux permettra de préciser les relations entre les différents lieux d'écriture en jeu et d'établir plus nettement les liens avec les données géopolitiques et patrimoniales.

Quant à la ville de Lyon et son entourage, on relève une proximité évidente des éléments oïliques présents avec le modèle de la scripturalité parisienne qui s'est construite à travers la deuxième moitié du $13^{\mathrm{e}}$ siècle (Glessgen 2017). Il faut toutefois prendre en considération aussi la proximité entre ce modèle, propagé ensuite par la chancellerie royale, et les scriptae de la région d'Orléans qui entretiennent un lien plus immédiat avec Lyon que Paris, par la voie de la Loire. Il serait par conséquent important de préciser la nature de l'écrit oïlique de la région de Lyon afin d'établir plus précisément les voies par lesquelles les modèles concrets se sont implantés en territoire francoprovençal. Comme nous l'avons déjà évoqué, il n'est pas surprenant que les protagonistes de l'écrit à Lyon se soient orientés vers le Bassin parisien qui était le seul réseau urbain ecclésiastique et princier plus important que le leur entre l'Atlantique et la Méditerranée et non vers une périphérie géopolitique du domaine d'oïl (comme la Bourgogne ou la Franche-Comté). De manière très concrète s'ajoute bien entendu l'emprise politique de la couronne sur Lyon, instaurée à partir de 1292 et bien établie à partir de 1312/1320.

\subsection{Encore le Corpus juris civilis}

Le cheminement de notre analyse nous a permis d'entrevoir une ligne de force jusqu'ici négligée dans le processus d'élaboration scripturale du francoprovençal. En fait, le plus ancien texte francoprovençal que nous connaissons est la Somme du code (ou Codi), traduction abrégée de la première partie du Codex juris civilis, un texte d'une certaine importance (51 feuillets) et, d'après les résultats de notre analyse, pratiquement exempt d'éléments oïliques. ${ }^{91}$ Il n'est pas exclu que sa scripta dauphinoise ait été en dialogue avec des modèles occitans, hypothèse que nous étudierons de plus près, une fois achevée l'intégration des corpus de Meyer (1909) et de Brunel (1926; 1952) dans les Documents linguistiques.

documents de la Suisse, mais sont absents des actes lyonnais (cf. Carles/Glessgen en préparation).

91 Cf. supra $\mathrm{n} .73$ le commentaire concernant les formes non précédées de palatale; quant à ' $\mathrm{A}$ [ précédé de palatale, nous supposons que la variance des issues dans le Codi s'explique par le fait que le changement de ['ic] en ['ia] est encore en cours vers 1200 (cf. supra n. 88). 
En attendant, la tradition textuelle de la Somme du code est facilement identifiable grâce aux excellentes synthèses de Frédéric Duval (2016) : la version francoprovençale repose non pas sur la version latine originelle, mais sur une retraduction latine du Codi occitan. Ce dernier réunit vers 1149/1160, sans doute à Saint-Gilles près d'Arles, les neuf premiers livres du Code de Justinien dans une élaboration textuelle et linguistique très notable, finement analysée par Johannes Kabatek (2005, 123-160 : " eine Innovation und eine große kulturelle Leistung »). Le texte occitan fut retraduit en latin au début du $13^{\mathrm{e}}$ siècle par Richard de Pise et un manuscrit de cette version semble avoir été amené peu après à la Grande Chartreuse : «le chancelier de Saint-Gilles Pierre Fouquois, successeur de Raoul de Saint-Gilles (auteur présumé du Codi selon André Gouron), s’est retiré dans ce monastère à la fin de sa vie en y amenant quelques livres » (Duval 2016). La version latine fut alors traduite en francoprovençal, sans doute également peu après le déplacement du chancelier dans le cadre du chapitre cathédral de Grenoble. Étant donné la remontée du droit romain vers le Nord, le long du Rhône, et la diffusion du notariat public, ce texte avait désormais un public parmi les laïcs :

«la traduction était en priorité confectionnée pour des lecteurs très peu frottés de latin. Royer et Thomas pensent qu'elle était plutôt destinée aux seigneurs féodaux, souvent appelés à arbitrer des cas litigieux. Elle pouvait également intéresser des marchands et des bourgeois fortunés, intéressés à la gestion de leurs biens. [...] La traduction abrège volontiers : les formules jugées inutiles sont supprimées et le discours gagne en concision en passant au francoprovençal. Certaines realia sont adaptées au lecteur dauphinois : ainsi l'huile d'olive se change en huile de noix» (Duval 2016, en renvoyant à Royer/Thomas 1929).

Le seul manuscrit conservé semble avoir été réalisé pendant la première moitié du $13^{\mathrm{e}}$ siècle pour le seigneur d'Uriage, près de Grenoble, et il est resté jusqu'au $20^{\mathrm{e}}$ siècle dans la collection du château. La copie comporte quelques erreurs, mais elle est rédigée avec soin et a connu deux «campagne[s] de correction » (Duval 2016).

Il s'agit donc d'un texte significatif, avec un vocabulaire et une syntaxe élaborés ayant connu une diffusion certaine dans les milieux concernés. Le cas du francoprovençal rejoint ainsi celui de l'occitan, de l'espagnol et du français où la réception du droit romain et les traductions du Corpus juris civilis ont considérablement contribué à l'élaboration scripturale des langues vernaculaires. Pour l'occitan et l'espagnol, ce processus a été retracé en détail par Johannes Kabatek (2005). Pour le français, Frédéric Duval (2016) et nous-même (Glessgen 2017) avons pu montrer qu'il en avait été de même. Le cas du francoprovençal en revanche n'a jamais été rapproché de ce phénomène d'élaboration à dimension européenne. Il est certain qu'en français et en espagnol, les traductions du Corpus 
juris civilis ont été suivies d'un développement scriptural sans pareil. En occitan, elles ont eu un impact sur trois ou quatre siècles, alors que la scripturalité du francoprovençal médiéval n’a pu se développer que pendant un siècle et demi. Pour chaque langue, les conditions socilinguistiques sont différentes mais le parallélisme reste néanmoins flagrant.

\subsection{La double ascendance du francoprovençal dans le Lyonnais}

Si la Somme du code peut assumer le rôle d'emblème fondateur de la scripta francoprovençale, il est vrai que Grenoble ne se développe pas ultérieurement comme centre de la scripturalité vernaculaire. Nous ne connaissons en dehors de la Somme du code que peu de textes de cette région montagnarde; le seul ensemble important étant les Comptes consulaires de Grenoble (1338/1339). ${ }^{92}$

Par la suite, l'élaboration scripturale du francoprovençal trouve son épicentre à Lyon. ${ }^{93}$ Cette métropole ouverte vers la Méditerranée et donc aussi vers l'espace occitan disposait de l'autonomie socio-économique et culturelle nécessaire pour poursuivre de manière plus systématique à l'aide de sa scripturalité l'idée d'une langue identitaire et particulière. Dans un sens, le phénomène rappelle les élaborations scripturales de type urbain qui caractérisent à cette même époque les villes italiennes de Gênes, Milan, Venise, Naples ou Palerme. Lyon joue en quelque sorte de nouveau pour le domaine francoprovençal le rôle central qu'elle a dû jouer dans la formation même de la langue. Si Lyon peut être considérée comme l'épicentre génétique du francoprovençal (cf. supra 1.2), elle semble en avoir été aussi l'épicentre sociolinguistique entre le milieu $d u 13^{\mathrm{e}}$ et la fin du $14^{\mathrm{e}}$ siècle.

Dans le détail, l'élaboration du francoprovençal à Lyon demande toutefois une attention particulière. Nous avons vu qu'avant le début de la francisation vers 1360/1380, les issues francoprovençales dans les documents lyonnais sont presque généralisées, même si elles ne sont jamais absolues. Pour -ATu tout comme

92 La thèse d'André Devaux (1892) comporte, en dehors des comptes consulaires, un testament de la région de Grenoble (1275) ainsi que trois courts textes provenant de Vienne (une charte loi de 1276 [Les usages du mistral des comtes de Vienne, 12 p.] les comptes consulaires de Vienne 1389 [6 p.] et un inventaire de 1403 [Leyde de Vienne]). Par ailleurs, il ne faut pas s'attendre à de grandes découvertes archivistiques pour cette région.

93 Gonon souligne que le Forez ne disposait pas d'une tradition de l'écrit francoprovençal propre : «n'usèrent du francoprovençal que les clercs originaires ou habitants des Montagnes du Matin, les Monts du Lyonnais, où l'influence de Lyon était, comme elle reste, prédominante » (1974, XXIVs.). 
pour -ATA non précédés de palatale le type «a〉 correspond à 95\% des occurrences contre $5 \%$ du type oïlique $\left\langle\right.$ e.${ }^{94}$ Cela reste un pourcentage important, mais il faut également prendre en considération les issues après palatale qui sont, dans certains cas, fortement francisantes (notamment pour -ARE avec 95 occ. de 〈ier> contre seulement 4 de «(i)ar», cf. supra 7.2). S’ajoute la ressemblance évidente des actes francoprovençaux avec leurs équivalents oïliques aux niveaux textuels et phrastiques. Ces constats demandent une explication.

Il est en effet difficile de comprendre pourquoi des scribes médiévaux se seraient contraints à introduire des éléments français dans un texte francoprovençal sans aucune finalité pragmatique reconnaissable. Les auteurs comme les récepteurs des documents parlaient et comprenaient le francoprovençal et, dans un contexte géolinguistique francoprovençal, ils n'avaient nullement besoin de l'insertion du français. Il est également difficile d'imaginer que ces éléments aient été introduits pour des raisons de prestige - dans ce cas, les scribes auraient pu faire directement appel au français: la tradition textuelle du français, plus ancienne et plus développée, était naturellement connue des scribes de par la proximité géographique du domaine d'oïl et de par le caractère nomade de l'exercice concret de leur profession. Ajoutons que le tissu ecclésiastique - qui prenait une part décisive dans la formation des normes écrites et des scribes - se caractérisait à tout moment par une grande mobilité.

Si la part langagière minoritaire $d u$ français à l'échelle du texte peut paraître en surface secondaire par rapport au tissu textuel francoprovençal dominant, il serait néanmoins possible de supposer que dans l'ontogenèse, le modèle français est antérieur. Il nous semble en effet probable que les éléments (infra-)lexématiques français présents dans les textes essentiellement francoprovençaux ne sont non pas des «francisations » (cf. notamment Horiot 1972), mais les reliquats d'un processus d'élaboration qui prenait appui sur le modèle fourni par le français. Nous supposons ainsi que dans les textes en question, le francoprovençal ait utilisé les modèles textuels et phrastiques fournis par le français et qu'il se soit substitué en grande partie à celui-ci aux niveaux grapho-phonétique, lexical et morphologique au point d'atteindre une physionomie propre et une apparence « autochtone ». La langue se glisse en quelque sorte dans le moule scriptural préétabli par son voisin, dans un processus d'élaboration "par substitution ». Dans ce sens, les éléments français seraient des résidus de ce processus et non pas des francisations ponctuelles.

94 Pour -ATU : 1179 occ. de 〈a〉 contre 58 de 〈é〉 (1225-1364); pour -ATA 86 occ. de 〈a〉 contre 5 de 〈és (1305-1385) ; cf. supra 6.1. 
Ce raisonnement n'a rien de surprenant si l'on considère que les élaborations écrites ne se font jamais sans modèle pré-existant et que, par exemple, la mise à l'écrit du français a certainement profité à son tour du modèle anglo-saxon (Glessgen 2017). Il permet en outre de fournir une explication rationnelle à un phénomène toujours souligné mais pour lequel on n'a jamais donné d'explication cohérente et donc satisfaisante.

Entendons-nous : il est bien probable que l'un ou l'autre manuscrit de la Somme du code grenoblois soit venu à la connaissance, directe ou indirecte, de quelque scribe travaillant à Lyon. Il serait cohérent de supposer que le modèle du Code justinien ait pris part dans l'élaboration scripturale de la métropole rhodanienne. Mais les actes produits à Lyon s'inscrivent intégralement dans d'autres genres textuels, pour lesquels les modèles vernaculaires pré-existants étaient français. Nous supposons ainsi une coprésence de deux modèles complémentaires dans la trajectoire du francoprovençal : la tradition de la Somme du code d'une part et celle des actes oïliques d'autre part. Il ne s'agirait donc pas de l'élaboration pleinement «autochtone» d'une scripta vernaculaire, mais encore moins de l'importation d'une scripta étrangère comme en Suisse romande. Dans le domaine des textes documentaires, on peut considérer en ligne générale que l'émergence du francoprovençal à l'écrit a suivi sur le territoire de la France les mêmes lignes de force que celle de ses langues voisines pendant le demi-millénaire compris entre le $9^{\mathrm{e}}$ et la fin du $14^{\mathrm{e}}$ siècle.

\subsection{L'élaboration secondaire du francoprovençal en Suisse}

Comme nous l'avons vu, en Suisse les scribes et leurs commanditaires choisissent de ne pas mettre en place une forme écrite de leur langue maternelle, mais d'emprunter le français (régional) en tant que langue écrite, tout en l'adaptant par l'insertion d'éléments autochtones. Ce choix peut paraître surprenant, surtout en contraste avec Lyon et la région de Bourg-en-Bresse où le francoprovençal était bien présent à l'écrit. Les raisons ne résidaient certainement pas dans une meilleure compétence du français à l'oral en Suisse. Il faut plutôt supposer qu'au contraire les scribes de Lyon étaient mieux formés et qu'ils étaient plus nombreux qu'à Neuchâtel, Fribourg ou Genève. Mais ce qui nous semble être décisif, ce n'est pas tant le niveau de langue des scribes, qui connaissaient en principe partout autant le latin que le français, que des mobiles sociologiques.

Si Müller et Marguerat ont évoqué la conscience identitaire de Fribourg (cf. supra 1.3.3), cet argument est encore bien plus valable pour Lyon dont l'autonomie économique était nettement supérieure. Le choix du français comme langue vernaculaire «support» en Suisse garantissait une bonne communication écrite 
entre les territoires francoprovençal et oïlique de part et d'autre du Jura, qui étaient dans de nombreux cas gérés par les mêmes seigneurs ou les mêmes propriétaires ecclésiastiques. Il s’ajoute d'un point de vue interne que le français, bien qu'étant une langue distincte ne s'éloignait pas autant du francoprovençal que d'autres langues. Une charte rédigée en français, parsemée de lexèmes francoprovençaux et lue à haute voix par un locuteur francoprovençal qui introduisait quelques adaptations phonétiques devait rester compréhensible aussi à un auditoire non francophone. Les scribes ont donc substitué l'ancienne langue écrite, le latin, qui n'était plus suffisamment compris par le public laïc auquel s'adressaient ces documents, par une nouvelle langue écrite de prestige, le français, qui, bien que langue différente de la langue héréditaire locale, parvenait à répondre à l'exigence de compréhensibilité.

Il nous semble enfin probable que les scriptae francoprovençalisantes de Suisse se soient inspirées de trois sources: (i) de la tradition pré-textuelle du dialogue entre francoprovençal et latin, (ii) des modèles textuels oïliques sur lesquels elles ont pris appui, et enfin (iii) des textes francoprovençaux de France dont la diffusion se place quelques décennies plus tôt et qui étaient certainement connus en Suisse. N'oublions pas dans ce contexte qu'en Suisse, on trouve de nombreuses chartes alors qu'en France, le francoprovençal reste cantonné aux documents de gestion, ce qui a pu ultérieurement motiver les scribes suisses à favoriser un modèle français. Ajoutons enfin que le paramètre géographique n'est pas mathématiquement déterminant en cela puisque la ville la plus excentrée, Fribourg, a développé la forme la plus marquée du point de vue francoprovençal. Il sera difficile de reconstituer les réseaux de dépendance étant donné la grande diversité des choix scripturaux, mais, là encore, une trajectoire générale devient reconnaissable.

\subsection{Le phénomène de la mixité}

À travers notre analyse scriptologique, la coprésence des scriptae oïlique et francoprovençale s'est avérée, comme l'on pouvait s'y attendre, être le facteur le plus saillant dans la physionomie des données. Si les issues francoprovençales dans les contextes non précédés de palatale restent assez généralisées en France jusqu'en 1360/1380, elles ne le sont pas dans tous les environnements après palatale. Par ailleurs, après ce moment, les formes interagissent fortement avec les correspondants oüliques.

En revanche, si la part francoprovençale est relativement faible dans les scriptae suisses, le cumul des différents éléments (infra-)lexématiques francoprovençaux crée une certaine densité et une certaine continuité dans les documents, 
comme nous l'avons vu dans les deux chartes présentées auparavant. Par ailleurs, si les éléments francoprovençaux en Suisse sont en ligne générale moins marqués, ils peuvent devenir majoritaires dans le cas de certaines configurations étymologiques particulières comme dans le cas de -ALE où la forme héréditaire francoprovençale coïncide avec une variante endémique en domaine d'oïl (cf. supra 5.6). Ajoutons, en prenant appui sur nos premiers sondages lexicologiques, que la présence d'un vocabulaire francoprovençal, aux plans formel et sémantique, est d'une grande constance dans les actes suisses. Étant donné que ce type de marquage régional peut ne pas interférer avec l'harmonie de la physionomie scripturale $e^{95}$ - les lexèmes francoprovençaux peuvent en effet adopter une logique graphématique oïlique -, il connaît moins de frein dans son insertion. Il s'agissait ainsi pour les scribes de souligner de manière reconnaissable la provenance des documents d'une région ou d'un endroit donnés, et les éléments francoprovençaux remplissent une fonction non seulement pragmatique mais aussi constitutive de leur scripta régionale.

On insistera toutefois sur le fait que nous sommes en face d'une interaction entre deux langues qui se place strictement au niveau de l'écrit et que la scripta n'entend à aucun moment refléter la genèse d'une nouvelle langue mixte à vocation orale.

Le degré de malléabilité que nous avons pu observer dans la compositionnalité des deux langues à l'écrit est surprenant. Les pourcentages des issues en opposition varient non seulement très fortement dans le temps et selon les régions et lieux d'écriture, mais aussi selon les environnements phonétiques et les configurations étymologiques. Nous avons également vu qu'une même région ou un même lieu d'écriture ne s'inscrit nullement dans une logique uniforme et cohérente. Les contradictions sont notables à tous les niveaux, même si l'analyse quantificative et structurelle montre aussi que les marques francoprovençales, examinées dans leur ensemble, répondent - malgré les contradictions décelées à une logique de système et ne sont pas les effets aléatoires et chaotiques de choix individuels.

La mixité est ainsi un élément définitoire de la scripturalité francoprovençale. C'est aussi en cela que réside sa grande particularité et son intérêt pour les études scriptologiques en général. Le cas du francoprovençal souligne en effet le caractère puissant et déterminant des processus interactifs en jeu au moment de l'élaboration d'une langue écrite. Outre l'oralité, qui reste déterminante, nous pouvons tracer ici avec précision la part que prennent différents modèles écrits

95 Ce que nous avons appelé « harmonie textuelle » à plusieurs reprises dans Carles (2011, 528 ; 538-540). 
dans la constitution d'une scripta donnée. Les phénomènes sont plus apparents que dans le dialogue entre deux variétés régionales d'un même diasystème. Grâce aux observations qui sont possibles dans cette configuration " réactive », il sera ainsi possible d'appréhender sur une nouvelle base méthodologique la compositionnalité interne des scriptae romanes "autochtones » et le dialogue qui s'instaure entre différentes régions et centres scripturaux.

\subsection{La contrastivité des scriptae francoprovençales et françaises}

De manière très générale, notre étude a pu illustrer qu'il est indispensable de prendre en considération les scriptae oïliques pour appréhender de manière adéquate les scriptae francoprovençales puisque les deux systèmes se trouvent en interaction constante. Autrement dit, ne pas prendre en considération le domaine français dans les études sur le francoprovençal médiéval nous semble être une erreur méthodologique. Nous pensons en outre que la prise en considération du francoprovençal permet d'accentuer la description du français médiéval et ouvre aussi de nouvelles perspectives dans ce sens.

Il est certain que les particularités scripturales que nous avons pu relever à l'intérieur même du domaine d'oïl sont avant tout caractéristiques du tissu scriptologique français. Cela concerne notamment le 〈-t〉 graphique final en Picardie ou les issues du type sei en Champagne/Lorraine ou encore la position particulière de la chancellerie royale de Paris qui forme un îlot homogène pour le paramètre à l'étude, tout comme pour beaucoup d'autres paramètres (cf. Glessgen 2017, Glessgen/Videsott 2017). La contrastivité avec les données provenant du territoire francoprovençal n'a pas été déterminante dans ces observations, mais elle a néanmoins permis d'appréhender les phénomènes dans une optique plus large. Leur identification et leur caractérisation deviennent plus nettes si l'on considère aussi les scriptae du domaine francoprovençal, qui sont inséparables, toujours pour la même raison d'interaction constante, du tissu scriptologique d'oïl.

Dans cette même logique, le traitement de 'A[ a permis de montrer que l'analyse scriptologique sur un corpus aussi vaste clarifie très sensiblement le paysage de l'écrit français, son évolutivité et sa physionomie géolinguistique. Nous avons ainsi pu préciser la grande dépendance des évolutions concrètes et des notations graphiques des issues de 'A[ avec l'environnement phonétique et nous avons pu appréhender la part contingente de certains choix scripturaux.

Un dernier aspect pour lequel la contrastivité oïl-francoprovençal s'avère déterminante, même si nous ne l'avons exploitée que partiellement dans ce cadre, concerne les choix plus ou moins individuels des différents lieux d'écriture. Notre 
objectif dans la présente étude a été de dresser un cadre plus général permettant dans un deuxième temps d'évaluer la position précise de tout acte et de tout rédacteur sur le continuum scriptural entre les deux langues en jeu et entre les pôles de la supra-régionalité et du marquage local. Une telle étude est particulièrement porteuse dans le cas de Fribourg, étude poursuivie en détail par Lorraine Fuhrer. Tout en s'inscrivant dans les logiques de la scripturalité suisse et de son dialogue avec les scriptae oïliques sud-orientales, Fribourg est le lieu d'écriture se rapprochant le plus de la physionomie de la scripta francoprovençalisante de France. La position particulière de la scripta fribourgeoise en Suisse est connue depuis longtemps, mais l'approche comparatiste - combinée à l'étude de détail permettra de mieux cerner son identité physionomique intermédiaire dans le triangle scriptologique francoprovençal de Suisse, francoprovençal de France et français régional.

\section{7 Épilogue}

En conclusion, si pour l'époque moderne et contemporaine, concevoir le francoprovençal comme une langue non standardisée et éclatée en de multiples sousensembles voire variétés locales est certainement justifié, il faut considérer différemment la situation de l'écrit médiéval. Quand, vers la fin du $14^{\mathrm{e}}$ siècle, la pression externe du français a mis un terme précoce aux processus d'élaboration scripturaux d'un demi-millénaire, ceux-ci étaient bien avancés et avaient considérablement enrichi la mosaïque de la Romania médiévale. Dans ce sens, nous sommes convaincus que la scriptologie moderne sur base de corpus peut fournir un apport rendant justice à cette langue qui nous livre « a real linguistic testbed, exhibiting many of the paths of development possible in western Romance languages » (Kristol 2016, 350).

\section{Bibliographie}

Aebischer, Paul, Chrestomathie franco-provençale : recueil de textes franco-provençaux antérieurs à 1630, Bern, Francke, 1950.

Ammann-Doubliez, Chantal, «La Première Collection des lois » de Fribourg en Nuithonie, Basel, Schwabe, 2009.

Ammann-Doubliez, Chantal/Müller, Wulf, L'ordonnance fribourgeoise de 1363 sur les moulins, in : Bouvier, Jean-Claude/Gourc, Jacques/Pic, François (edd.), Mélanges offerts à Xavier Ravier, Toulouse, CNRS, 2003.

Aquino-Weber, Dorothée/Cotelli, Sara/Kristol, Andres (edd.), Sociolinguistique historique du domaine galloroman. Enjeux et méthodologies, Bern et al., Lang, 2009. 
ARTEM = Chartes originales antérieures à 1121 conservées en France, publication en ligne, 2010, $<w w w . c n$-telma.fr/originaux> [dernière consultation 15/02/2018].

Ascoli, Graziadio Isaia, Schizzi franco-provenzali, Archivio glottologico italiano 3 (1878), 61-120.

Berchtold, Elisabeth, Dictionnaire de l'ancien francoprovençal. Conception d'un projet lexicographique et réalisation sectorielle [échantillon : lettre F-], Universités de Neuchâtel et de Lorraine, thèse, ms., 2018.

Bourciez, Edouard/Bourciez, Jean, Phonétique française. Etude historique, Paris, Klincksieck, 1967.

Brasseur, Patrice, Les infinitifs issus du latin «-are » dans les parlers bas-normands, Revue de linguistique romane 82 (2018), 93-134.

Brun, Auguste, Recherches historiques sur l'introduction du français dans les provinces du Midi, Paris, Champion, 1923.

Brunel, Clovis, Les plus anciennes chartes en langue provençale. Recueil des pièces originales antérieures au XIIIe siècle. Publiées avec une étude morphologique, Paris, Picard, 1926.

Brunel, Clovis, Les plus anciennes chartes en langue provençale. Recueil des pièces originales antérieures au XIIIe siècle. Supplément, Paris, Picard, 1952.

Bürli, Riccarda, Lieux d'écriture dans les domaines d'oïl et francoprovençal : paramètres linguistiques, mémoire de MA, Zürich, 2017.

Carles, Hélène, Ad fontes. Französische Texte des Mittelalters, 2008-2016 (<www.adfontes.uzh. ch/3200.php>).

Carles, Hélène, L'émergence de l'occitan pré-textuel. Analyse linguistique d'un corpus auvergnat (IXe-XIe s.), Strasbourg, ÉLiPhi/SLR, 2011.

Carles, Hélène, Trésor galloroman des origines (TGO). Les trajectoires étymologiques et géolinguistiques du lexique galloroman en contexte latin (ca 800-1120), Strasbourg, ÉLiPhi, 2017.

Carles, Hélène/Glessgen, Martin, Le vocabulaire régional dans la langue d'oïl orientale et sudorientale au Moyen Âge, en préparation.

Chambon, Jean-Pierre, La francoprovençalité de Cluny au début du XIVe siècle (indices tirés de l'anthroponymie), in : Horiot, Brigitte/Schafroth, Elmar/Simoni-Aurembou, Marie-Rose (edd.), "Je parle, donc je suis ... de quelque part ». Mélanges offerts au professeur Lothar Wolf, Lyon, Centre d'Études linguistiques Jacques Goudet, H.S. 2, Université Lyon 3 Jean Moulin, 2005, 247-254.

Chambon, Jean-Pierre/Greub, Yan, Histoire des dialectes dans la Romania : Galloromania, in : Ernst, Gerhard/Gleßgen, Martin-Dietrich/Schmitt, Christian/Schweickard, Wolfgang (edd.), Romanische Sprachgeschichte / Histoire linguistique de la Romania, vol. 3, Berlin/New York, De Gruyter, 2008, 2499-2520.

Chambon, Jean-Pierre/Olivier, Philippe, L'histoire linguistique de l'Auvergne et du Velay: notes pour une synthèse provisoire, Travaux de Linguistique et de Philologie 38 (2000), 83-153.

Dees, Anthonij, Atlas des formes et des constructions des chartes françaises au 13e siècle, Tübingen, Niemeyer, 1980.

Dees, Anthonij, Dialectes et «scriptae » à l'époque de l'ancien français, Revue de linguistique romane 49 (1985), 87-117.

Devaux, André, Essai sur la langue vulgaire du Dauphiné septentrional au Moyen Âge, Paris/ Lyon, Welter/Auguste Cote, ${ }^{1} 1892$ [Genève, Slatkine, ${ }^{2} 1968$ ].

Devaux, André/Ronjat, Jules, Comptes consulaires de Grenoble en langue vulgaire (1338-1340), Revue des langues romanes 55 (1912), 145-382. 
DocLing = Documents linguistiques galloromans. Édition électronique, dirigée par Martin Glessgen, en collaboration avec Hélène Carles/Frédéric Duval/Paul Videsott, ${ }^{3} 2016$, <www.rose. uzh.ch/docling>.

Duraffour, Antonin, La langue des comptes syndicaux de Châtillon-les-Dombes (1385-1500), Bourg, Imprimerie du Courrier de l'Ain, 1925.

Duraffour, Antonin, En marge du Terrier de Chazelles, in : Gardette, Pierre, et al. (edd.), Mélanges J. Saunier, Lyon, Facultés catholiques, 1944, 219-236.

Durdilly, Paulette, Nouveaux fragments du livre de comptes d'un marchand lyonnais, Revue de linguistique romane 28 (1964), 375-407.

Durdilly, Paulette, Extraits du livre de comptes de J. Panczus, receveur de tailles à Lyon, in : Mélanges de linguistique et de philologie romanes offerts à Pierre Gardette, Paris, Klincksieck, 1966, 135-146.

Durdilly, Paulette, Écrivait-on encore en dialecte à Lyon vers la fin du XIVe siècle ?, Revue de linguistique romane 36 (1972), 376-383.

Durdilly, Paulette, Documents linguistiques de la France (série francoprovençale). Documents Linguistiques du Lyonnais (1225-1425), Paris, CNRS, 1975.

Durdilly, Paulette, Quelques témoins de la langue écrite à Lyon au XVe siècle, Vox Romanica 40 (1981), 104-111.

Duval, Frédéric, Miroir des classiques-2. Les traductions galloromanes du « Corpus juris civilis ». Répertoire électronique, 2016, <http://elec.enc.sorbonne.fr/miroirdesclassiques> [dernière consultation 15/02/2018].

Englebert, Annick, Introduction à la phonétique historique du français, Bruxelles, De Boeck/ Duculot, 2009.

Fouché, Pierre, Phonétique historique du français, 3 vol., Paris, Klincksieck, 1952.

Fréchet, Claudine, Les Comptes de Thoire et Villard d'après des registres allant de 1361 à 1371. AD Côte d'Or B 8240, in : Aux racines du francoprovençal. Actes du Centre d'études francoprovençales «René Willien », Saint-Nicolas, 20-21 décembre 2003, Aoste, Région autonome de la Vallée d'Aoste, 2004, 71-80.

Fuhrer, Lorraine, Édition des plus anciens documents linguistiques de Fribourg, en préparation.

Gardette, Pierre, Les textes non littéraires et le vocabulaire francoprovençal / Explication de quelques mots extraits d'un tarif de péage de Belleville-sur-Saône au XIVe siècle / Projet d'un glossaire du vieux francoprovençal, Revue de linguistique romane 26 (1962), 363-394.

Gardette, Pierre, Le francoprovençal écrit en Lyonnais et en Forez au moyen âge, Revue de linguistique romane 32 (1968), 70-99.

Gardette, Pierre, Le francoprovençal, son histoire, ses origines, in : Moignet, Gérard/Lassalle, Roger, Actes du 5e congrès international de langue et littérature d'oc et d'études francoprovençales, Paris, Les Belles Lettres, 1974, 294-305.

Gärtner, Kurt/Holtus, Günter (edd.), Beiträge zum Sprachkontakt und zu den Urkundensprachen zwischen Maas und Rhein, Trier, Trierer Historische Forschungen, 1995.

Gärtner, Kurt/Holtus, Günter (edd.), Urkundensprachen im germanisch-romanischen Grenzgebiet, Mainz, Zabern, 1997.

Gärtner, Kurt/Holtus, Günter (edd.), Überlieferungs- und Aneignungsprozesse im 13. und 14. Jahrhundert auf dem Gebiet der westmitteldeutschen und ostfranzösischen Urkundenund Literatursprachen, Trier, Kliomedia, 2005.

Gasca Queirazza, Giuliano, Individuazione di elementi di francoprovenzale nelle carte e documenti latini medievali della Val d'Aosta, in : Aux racines du francoprovençal. Actes du Centre 
d'études francoprovençales «René Willien », Saint-Nicolas, 20-21 décembre 2003, Aoste, Région autonome de la Vallée d'Aoste, 2004, 87-94.

Gauthier, Jules, Les documents franc-comtois des archives de Neuchâtel (Suisse), Besançon, Imprimeries Dodivers, 1880.

Gauthier, Jules, Recueil de quarante-huit chartes françaises de 1227 à 1280 pour servir à l'étude du dialecte franc-comtois, in : Académie de Besançon (ed.), Mémoires et documents pour servir à l'histoire de la Franche-Comté, Besançon, Jacquin, 1900, 521-574.

Gavillet, Bernadette, Anciens documents en langue française conservés dans les archives des cantons de Vaud et de Genève, Neuchâtel, ms., 2011.

Glessgen, Martin, Les lieux d'écritures dans les chartes lorraines du XIIle siècle, Revue de linguistique romane 72 (2008), 413-540.

Glessgen, Martin, Linguistique romane. Domaines et méthodes en linguistique française et romane, Paris, Colin, ${ }^{2} 2012\left({ }^{1} 2007\right)$ (= 2012a).

Glessgen, Martin, Trajectoires et perspectives en scriptologie romane, Medioevo romanzo 36 (2012), 5-23 (= 2012b).

Glessgen, Martin, L'écrit documentaire et le projet des «Plus anciens documents linguistiques de la France », in : Trotter, David (ed.), Manuel de la philologie de l'édition, Berlin/Boston, De Gruyter, 2015, 267-295.

Glessgen, Martin, La régionalité lexicale au Moyen Âge : hypothèses, constats et perspectives, in : id./Trotter, David, La régionalité lexicale du français au Moyen Âge, Strasbourg, ÉLiPhi, 2016, 1-34.

Glessgen, Martin, La genèse d'une norme en français au Moyen Âge : mythe et réalité du "francien », Revue de linguistique romane 81 (2017), 313-398.

Glessgen, Martin, Les « documents linguistiques » de la France. Histoire, présent et perspectives d'un projet centenaire, in : Comptes rendus des séances de l'Académie des Inscriptions et Belles-Lettres (CRAI) 161/3 (Juillet à Octobre 2017), 2018, 1239-1270.

Glessgen, Martin/Tittel, Sabine, Le «Dictionnaire d'ancien gascon » électronique (DAGél), in : Antonelli, Roberto/Glessgen, Martin/Videsott, Paul (edd.), Atti del XXVIII Congresso internazionale di linguistica e filologia romanza (Roma, 18-23 luglio 2016), vol. 1, Strasbourg, SLR/ÉLiPhi, 2018, 799-813.

Glessgen, Martin/Tittel, Sabine, L'intégration du vocabulaire des « Documents linguistiques galloromans » dans le DEAFélectronique, en préparation.

Glessgen, Martin/Videsott, Paul, L'élaboration du lexique français médiéval - variation diatopique, sélection et changement lexicaux, Romanistisches Jahrbuch 68 (2017), 64-89.

Goebl, Hans, «Le Rey est mort, vive le Roy ». Nouveaux regards sur la scriptologie, Travaux de linguistique et de littérature 12:1 (1975), 145-210 (= 1975a).

Goebl, Hans, Qu'est-ce que la scriptologie ?, Medioevo Romanzo 2 (1975), 3-43 (= 1975b).

Goebl, Hans, «Verba volant, scripta manent ». Quelques remarques à propos de la « scripta » normande, Revue de linguistique romane 43 (1979), 344-399.

Goebl, Hans/Smečka, Pavel, L'interprétation dialectométrique des atlas « scripturaux » d'Antonij Dees, Revue de linguistique romane 80 (2016), 321-368.

Gonon, Marguerite, La vie religieuse en Forez au XIVe siècle et son vocabulaire d'après les testaments, Archivium latinitatis medii aevi 30 (1960), 233-286.

Gonon, Marguerite, La vie familiale en Forez au XIVe siècle et son vocabulaire d'après les testaments, Paris, Les Belles Lettres, 1961 [= Thèse Lettres Strasbourg, 1959].

Gonon, Marguerite, Glossaire forézien du 15e siècle, d'après les testaments, Revue de linguistique romane 28 (1964), 408-445 ; 29 (1965), 141-186, 314-358. 
Gonon, Marguerite, L'inventaire des biens d'un citoyen de Lyon en 1327, in : Mélanges de linguistique et de philologie romanes offerts à Monseigneur Pierre Gardette, Strasbourg/ Paris, Klincksieck, 1966.

Gonon, Marguerite, Documents linguistiques de la France (série francoprovençale). Documents linguistiques du Forez (1260-1498), Paris, CNRS, 1974.

Gonon, Marguerite, Latin, français et francoprovençal en Lyonnais et Forez à la fin du Moyen-Age, in : Centre d'études francoprovençales « René Willien » (ed.), Histoire linguistique de la Vallée d'Aoste du Moyen Age au XVIIIe siècle, Aoste, Région autonome de la Vallée d'Aoste, 1985, 48-52.

Gossen, Carl Theodor, Untersuchungen zur jurassischen Scripta, Vox Romanica 23 (1964), 321-354.

Gossen, Carl Theodor, La scripta bourguignonne et le francoprovençal, Revue roumaine de linguistique 10 (1965), 455-460.

Gossen, Carl Theodor, Französische Skriptastudien, Wien, Österreichische Akademie der Wissenschaften, 1967.

Gossen, Carl Theodor, Graphème et phonème : le problème central de l'étude des langues écrites du Moyen Âge, Revue de linguistique romane 32 (1968), 1-16.

Gossen, Carl Theodor, Considérations sur la « scripta » "para-francoprovençale », Revue de linguistique romane 34 (1970), 326-348.

Grübl, Klaus, La standardisation du français au Moyen Âge : point de vue scriptologique, Revue de linguistique romane 77 (2013), 343-383.

Grübl, Klaus, Varietätenkontakt und Standardisierung im mittelalterlichen Französisch. Theorie, Forschungsgeschichte und Untersuchung eines Urkundenkorpus aus Beauvais (1241-1455), Tübingen, Narr, 2014.

Grübl, Klaus, Ce que les chartes nous apprennent sur la variation et le changement linguistique au Moyen Âge : l'exemple de la déclinaison bicasuelle de l'ancien français, Revue de linguistique romane 79 (2015), 33-69.

Grüner, Laure, Contribution à l'histoire du lexique francoprovençal : structures et méthode rédactionnelle pour un dictionnaire étymologique [échantillon : lettre F-], ms. Universités de Neuchâtel et de Lorraine.

Guichard, Georges, et al., Chartes du Forez antérieures au XIVe siècle, Macon/Paris, Protat/ Klincksieck, 1933-1980.

Hafner, Hans, Grundzüge einer Lautlehre des Altfrankoprovenzalischen, Bern, Francke, 1955.

Hasselrot, Bengt, Les limites du francoprovençal et l'aire de « nostron », Revue de linguistique romane 30 (1966), 257-266.

Hilty, Gerold, Les plus anciens textes français et l'origine du standard, in : Knecht, Pierre/Marzys, Zygmunt/Destraz, Dominique (edd.), Écriture, langues communes et normes. Actes du Colloque tenu à l'Université de Neuchâtel (21-23 sept. 1988), Neuchâtel/Genève, Universités, 1993, 9-16.

Horiot, Brigitte, Pronoms et déterminatifs en ancien francoprovençal, in : Marzys, Zygmunt/ Voillat, François (edd.), Colloque de dialectologie francoprovençale (Neuchâtel, 23-27 sept. 1969), Neuchâtel, Faculté des Lettres/Genève, Droz, 1971, 125-150.

Horiot, Brigitte, Recherches sur la morphologie de l'ancien francoprovençal, Revue de linguistique romane 36 (1972), 1-74.

Horiot, Brigitte/Du Pouget, Marc, Un journal de recette du péage de Belleville au XVe siècle, Revue de linguistique romane 54 (1990), 93-142. 
Jeanjaquet, Jules, Un document inédit du français dialectal de Fribourg au XIVe siècle, in : Aus romanischen Sprachen und Literaturen. Festschrift Heinrich Morf zur Feier seiner fünfundzwanzigjährigen Lehrtätigkeit von seinen Schülern dargebracht, Halle, Niemeyer, 1905, 271-296.

Jud, Jakob, Observations sur le lexique de la Franche-Comté et du francoprovençal, in : Studies in French language and mediæval litterature presented du Professor Mildred K. Pope, Manchester, University Press, 225-240.

Kabatek, Johannes, Die Bolognesische Renaissance und der Ausbau romanischer Sprachen. Juristische Diskurstraditionen und Sprachentwicklung in Südfrankreich und Spanien im 12. und 13. Jahrhundert, Tübingen, Niemeyer, 2005.

Kihaï, Dumitru, Écriture et pouvoir au 13e siècle en Champagne : identification des principaux lieux d'écriture, Thèse de doctorat, Zürich/Strasbourg, 2011.

Koch, Peter, Pour une typologie conceptionnelle et médiale des plus anciens documents/monuments des langues romanes, in : Selig, Maria/Frank, Barbara/Hartmann, Jörg (edd.), Le passage à l'écrit des langues romanes, Tübingen, Narr, 1993, 39-81.

Kristol, Andres, À la découverte de l'ancien francoprovençal : le témoignage de la toponymie haut-valaisanne, in : Colligere atque tradere : études d'ethnographie alpine et de dialectologie francoprovençale. Mélanges offerts à Alexis Bétemps, Saint-Christophe, Aoste, BREL, Région autonome de la Vallée d'Aoste, 2003, 111-119.

Kristol, Andres, Sur les traces du francoprovençal prélittéraire : l'enseignement des toponymes d'origine francoprovençale dans la Romania submersa en Suisse occidentale, in : Aux racines du francoprovençal. Actes du Centre d'études francoprovençales «René Willien », Saint-Nicolas, 20-21 décembre 2003, Aoste, Région autonome de la Vallée d'Aoste, 2004, 23-38.

Kristol, Andres, Dialectologie synchronique et diachronique, disciplines complémentaires. Ce que les études dialectales en synchronie peuvent apporter à la linguistique diachronique, in : Ayres-Bennett, Wendy/Rainsford, Thomas M. (edd.), L'histoire du français : états des lieux et perspectives, Paris, Garnier, 2014, 137-157.

Kristol, Andres, Francoprovençal, in : Ledgeway, Adam/Maiden, Martin (edd.), The Oxford Guide to the Romance Languages, Oxford, University Press, 2016, 350-362.

La Chaussée (de), François, Initiation à la phonétique historique de l'ancien français, Paris, Klincksieck, 1989.

Lescuyer-Mondésert, Jeanne-Marie, Construction, entretien, réparation des fortifications aux XIIle et XIVe siècles dans les régions qui correspondent à l'actuel département de l'Ain, d'après les documents non littéraires, Bulletin philologique et historique du Comité des travaux historiques et scientifiques (1978), 165-181.

Lescuyer-Mondésert, Jeanne-Marie, Structure et entretien des étangs de Bresse et de Dombes à la fin du XIIle et au début du XIVe siècle, Art et histoire des pays de l'Ain (1979 [1980]), 18-49.

Lescuyer-Mondésert, Jeanne-Marie, Gippons et chapirons; estiveaulz et solars ; seya, futayne, cendal et autres « garnimenz » de la vie domestique, d'après le registre de Loyes et Villars, B8240, des Archives départementales de la Côte-d'Or, in : Dialectologie, histoire et folklore. Mélanges offerts à Ernest Schüle pour son 70e anniversaire, Bern, Francke, 1983, 220-230.

Lodge, Anthony, A Sociolinguistic History of Parisian French, Cambridge, Cambridge University Press, 2004.

Lodge, Anthony, La sociolinguistique historique et le problème des données, in : Aquino-Weber, Dorothée/Cotelli, Sara/Kristol, Andres (edd.), Sociolinguistique historique du domaine galloroman. Enjeux et méthodologies, Bern et al., Lang, 2009, 199-219. 
Lüdi, Georges, Mehrsprachige Rede in Freiburger Ratsmanualen des 15. Jahrhunderts, Vox Romanica 44 (1985), 163-188.

Lüdi, Georges, Un exemple de polyglossie : Fribourg i. Ue. aux XIVe/XVe siècles, in : Rencontres régionales. Actes du troisième Colloque régional de linguistique, Strasbourg 28-29 avril 1988, Strasbourg, USHS, 1989, 257-276.

Lusignan, Serge, Essai d'histoire sociolinguistique. Le français picard au Moyen Âge, Paris, Garnier, 2012.

Maffei Boillat, Stefania, Le Mariale lyonnais (Paris, BNF, fr. 818) : édition, traduction et étude linguistique, Strasbourg, ÉLiPhi, 2015.

Maffei Boillat, Stefania, Un censier vaudois du XIVe siècle en langue vulgaire, in : ead./Corbellari, Alain, L'aventure du sens. Mélanges de philologie provençale en l'honneur de François Zufferey, Strasbourg, ÉLiPhi, 2016, 123-146.

Mallet, Edouard, Chartes inédites relatives à la ville et au diocèse de Genève et antérieures à l'année 1312, Genève/Paris, Société d'Histoire et d'Archéologie de Genève, 1862.

Marguerat, Philippe, Documents linguistiques de la Suisse romande (XIIIe-XVe), Thèse dactylographiée de l'École des Chartes, 1960.

Marguerat, Philippe, Pratiques juridiques et usages linguistiques dans le domaine francoprovençal du XIIIe au XVle siècle, in : Marzys, Zygmunt/Voillat, François (edd.), Colloque de dialectologie francoprovençale (Neuchâtel, 23-27 sept. 1969), Neuchâtel/Genève, Faculté des Lettres/Droz, 1971, 151-172.

Marzys, Zygmunt, De la « scripta » au patois littéraire : à propos de la langue des textes francoprovençaux antérieurs au XIXe siècle, Vox Romanica 37 (1978), 193-213.

Marzys, Zygmunt, Une charte jurassienne inédite du début du XIVe siècle, in : Cerquiglini-Toulet, Jacqueline/Collet, Olivier (edd.), Mélanges de philologie et de littérature médiévales offerts à Michel Burger, Neuchâtel/Genève, Université/Droz, 1994, 139-151.

Marzys, Zygmunt/Scheurer, Rémy, Latin et langue vulgaire dans les chartes de Bellelay au XIVe siècle, in : Knoepfler, Denis (ed.), Nomen latinum, Neuchâtel/Genève, Faculté des Lettres/Droz, 1997, 299-316.

Marzys, Zygmunt/Voillat, François, Actes [du] Colloque de dialectologie francoprovençale, (Neuchâtel, 23-27 sept. 1969), Neuchâtel/Genève, Faculté des Lettres/Droz, 1971.

Matile, Georges-Auguste, Monuments de l'histoire de Neuchâtel publiés par les ordres et aux frais de Sa Majesté Frédéric-Guillaume IV, roi de Prusse, prince souverain de Neuchâtel et Valangin, 2 vol., Neuchâtel, Attinger, 1844-1848.

Meyer, Paul, Maître Pierre Cudrifin, horloger, et la ville de Romans, Romania 21 (1892), 39-49.

Meyer, Paul, Documents linguistiques du Midi de la France, Paris, Champion, 1909.

Müller, Wulf, A propos de la « scripta » fribourgeoise, Vox Romanica 40 (1981), 77-84.

Müller, Wulf, Le démonstratif « cellour » en ancien fribourgeois (XIVe siècle), in : Cahiers Louis Gauchat. Bulletin du Centre de dialectologie et d'étude du français régional 1 (1990), 13-28.

Müller, Wulf, Sur la morphologie de l'ancien fribourgeois (14e siècle), in : Combettes, Bernard/ Monsonego, Simone (edd.), Le moyen français, Paris, Didier, 1997, 131-141.

Müller, Wulf, Die Urkundensprache von Fribourg im 14. (und 15.) Jahrhundert, in : Gärtner, Kurt/ Holtus, Günter/Rapp, Andrea/Völker, Harald (edd.), Skripta, Schreiblandschaften und Standardisierungstendenzen, Trier, Trierer Historische Forschungen, 2001, 245-256.

Pfister, Max, "Scripta » et koinè en ancien français aux XIle et XIIle siècles ?, in : Knecht, Pierre/ Marzys, Zygmunt/Destraz, Dominique (edd.), Écriture, langues communes et normes. Actes du Colloque tenu à l'Université de Neuchâtel du 21 au 23 septembre 1988, Neuchâtel/ Genève, Faculté des Lettres/Droz, 1993, 17-41. 
Pfister, Max, Nordöstliche Skripten im Grenzbereich Germania-Romania vor 1300, in : Gärtner, Kurt/Holtus, Günter/Rapp, Andrea/Völker, Harald (edd.), Skripta, Schreiblandschaften und Standardisierungstendenzen, Trier, Trierer Historische Forschungen, 2001, 223-244.

Philipon, Édouard, Une page de l'histoire de Lyon pendant la guerre de Cent Ans. Le règlement fiscal de 1351, Lyon-Revue 5 (1883), 178-194 ; 228-247 ; 274-285.

Philipon, Édouard, Phonétique lyonnaise au XIVe siècle, Romania 13 (1884), 542-590.

Philipon, Édouard, Les bénéfices du Chapitre de Saint-Jean, à Saint-Germain-au-Mont-d'Or et à Poleymieux, d'après un terrier en dialecte lyonnais du XIIle siècle, Revue lyonnaise 9 (1885), 420-425.

Philipon, Édouard, Le dialecte bressan aux XIIIe et XIVe siècles, Revue des patois 1 (1887), 11-57.

Philipon, Édouard, Morphologie du dialecte lyonnais aux XIIle et XIVe siècles, Romania 30 (1901), 213-294.

Philipon, Édouard, Documents linguistiques du département de l'Ain, in : Meyer, Paul (ed.), Documents linguistiques du Midi de la France, Paris, Champion, 1909.

Philipon, Édouard, Les parlers du duché de Bourgogne aux XIIIe et XIVe siècles, Romania 39 (1910), 476-531.

Philipon, Édouard/Perrat, Charles, Le livre du vaillant des habitants de Lyon en 1388 : estimation des biens meubles et immeubles pour servir à l'assiette de la taille, Lyon, Impressions de M. Audin et cie., 1927.

Pierret, Jean-Marie, Phonétique historique du français et Notions de phonétique générale, Louvain-la-Neuve, Peeters, 1994.

Quaglia, Lucien/Theurillat, Jean-Marie/Schüle, Ernest, Les comptes de l'Hospice du Grand SaintBernard (1399-1477), Vallesia 28 (1973), 1-162 ; 30 (1975), 169-384.

Remacle, Louis, Le problème de l'ancien wallon, Liège, Faculté de Philosophie et Lettres, 1948.

Romaine, Suzanne, Socio-Historical Linguistics : its Status and Methodology, Cambridge, Cambridge University Press, 1982.

Romaine, Suzanne, Historical sociolinguistics : problems and methodology, in : Ammon, Ulrich/ Dittmar, Norbert/Mattheier, Klaus J./Trudgill, Peter (edd.), Sociolinguistics. An International Handbook of the Science of Language and Society / Soziolinguistik. Ein internationales Handbuch zur Wissenschaft von Sprache und Gesellschaft, vol. 2, Berlin, De Gruyter, 1988, 1452-1469.

Royer, Louis/Thomas, Antoine, La Somme du code : texte dauphinois de la région de Grenoble publié d'après un manuscrit du XIIle siècle appartenant à la bibliothèque du château d'Uriage, Paris, Imprimerie Nationale, 1929.

RSG = Ernst, Gerhard/Gleßgen, Martin-Dietrich/Schmitt, Christian/Schweickard, Wolfgang (edd.), Romanische Sprachgeschichte / Histoire linguistique de la Romania, 3 vol., Berlin/ New York, Mouton/De Gruyter, 2003-2008.

Salmon, Gilbert, Phonétique et graphies des XIIIe-XVe siècles en Lyonnais-Forez, Bulletin de la Faculté des Lettres de Mulhouse 9 (1978), 49-70.

Scheurer, Rémy/Morerod, Jean-Daniel/Andres, Kristol, Documents linguistiques de la Suisse romande II. Documents en langue française antérieurs à la fin du XIVe siècle conservés dans le canton de Neuchâtel, à paraître.

Schmitt, Christian, À propos de la formation linguistique du domaine francoprovençal, Revue de linguistique romane 41 (1977), 91-103.

Schüle (1975) $\rightarrow$ Quaglia/Theurillat/Schüle. 
Schüle, Ernest, Histoire et évolution des parlers francoprovençaux d'Italie (état des travaux et perspectives de recherches nouvelles), in : Lingue e dialetti nell'arco alpino occidentale. Atti del convegno internazionale di studio (Torino, 12-14 aprile 1976), Torino, Centro studi piemontesi, 1978, 127-140.

Schüle, Ernest/Scheurer, Rémy/Marzys, Zygmunt (edd.), Documents linguistiques de la Suisse romande, vol. 1: Documents en langue française antérieurs à la fin du XIV siècle conservés dans les cantons du Jura et de Berne, Paris, CNFS, 2002.

Stimm, Helmut, Studien zur Entwicklungsgeschichte des Frankoprovenzalischen, Mainz/Wiesbaden, Akademie der Wissenschaften und der Literatur/Steiner, 1952.

Stimm, Helmut, Altfrankoprovenzalische Übersetzungen hagiographischer lateinischer Texte aus der Handschrift der Pariser Nationalbibliothek fr. 818. I. Prosalegenden, Mainz/Wiesbaden, Akademie der Wissenschaften und der Literatur/Steiner, 1955.

Straka, Georges, Les sons et les mots. Choix d'études de phonétique et de linguistique, Paris, Klincksieck, 1979.

TGO $\rightarrow$ Carles (2017).

Tinner, Sarah, Les lieux d'écriture dans les chartes franc-comtoises du XIIIe siècle, mémoire de MA, Zürich, 2009.

Tinner, Sarah, Les lieux d'écriture dans les chartes de la Franche-Comté, ms. Zürich, 2011.

Trouillat, Joseph/Vautrey, Louis, Monuments de l'histoire de l'ancien évêché de Bâle, 5 vol., Porrentruy, Michel/Gürtler, 1852-1867.

Tuaillon, Gaston, La littérature en francoprovençal avant 1700, Grenoble, ELLUG - Université Stendhal, 2001.

Videsott, Paul, Les débuts du français à la Chancellerie royale : analyse scriptologique des chartes de Philippe III (1250-1285), Revue de linguistique romane 77 (2013), 3-50.

Videsott, Paul, Les plus anciens documents en français de la chancellerie royale capétienne (1241-1300). Présentation et édition, Strasbourg, ÉLiPhi, 2015 (= 2015a).

Videsott, Paul, Comment écrivait la chancellerie royale capétienne au XIIle siècle ? Un aperçu géo-quantitatif sur la base du Corpus des actes royaux vernaculaires du XIIIe siècle, Zeitschrift für romanische Philologie 131 (2015), 863-910 (= 2015b).

Vieillard, Jeanne, Le latin des diplômes royaux et chartes privées de l'époque mérovingienne, Paris, Champion, 1927.

Villeneuve, Etienne de/Guigue, Marie-Claude, Cartulaire municipal de la ville de Lyon : privilèges, franchises, libertés et autres titres de la Commune : recueil formé au XIVe siècle, Genève, Mégariotis, 1978.

Vitali, David, Mit dem Latein am Ende? Volkssprachlicher Einfluss in lateinischen Chartularen aus der Westschweiz, Bern, Lang, 2007.

Völker, Harald, Skripta und Variation. Untersuchungen zur Negation und zur Substantivflexion in altfranzösischen Urkunden des Grafschaft Luxemburg (1237-1281), Tübingen, Niemeyer, 2003.

Vurpas, Anne-Marie, Frankoprovenzalische Skriptae, in : Holtus, Günter/Metzeltin, Michael/ Schmitt, Christian (edd.), Lexikon der Romanistischen Linguistik (LRL), vol. 2/2: Die einzelnen romanischen Sprachen und Sprachgebiete vom Mittelalter bis zur Renaissance, Tübingen, Niemeyer 1995, 389-405.

Werro, Romain/Berchtold, Jean/Gremaud, Jean, Recueil diplomatique du canton de Fribourg, Fribourg, Piller, 1839-1877.

Zink, Gaston, Phonétique historique du français, Paris, Presses universitaires de France, ${ }^{3} 1991$. 
Zufferey, François, Langues et parlers, in : Paravicini Bagliani, Agostino/Felber, Jean-Pierre/ Morerod, Jean-Daniel/Pasche Véronique (edd.), Les pays romands au Moyen Age, Lausanne, Payot, 1997, 392-404.

Zufferey, François, Robert de Boron et la limite nord du francoprovençal, Revue de linguistique romane 70 (2006), 431-469. 